\title{
INVARIANT THEORY OF FINITE GROUP ACTIONS ON DOWN-UP ALGEBRAS
}

\author{
E. KIRKMAN, J. KUZMANOVICH AND J.J. ZHANG
}

\begin{abstract}
We study Artin-Schelter Gorenstein fixed subrings of some ArtinSchelter regular algebras of dimension 2 and 3 under a finite group action and prove a noncommutative version of the Kac-Watanabe and Gordeev theorem for these algebras.
\end{abstract}

\section{INTRODUCTION}

This paper continues our project of extending classical invariant theory to a noncommutative context. Throughout, let $A$ be an Artin-Schelter regular (AS regular, for short) algebra over an algebraically closed field $k$ of characteristic zero, let $\operatorname{Aut}(A)$ be the group of graded automorphisms of $A$, and let $H$ be a finite subgroup of Aut $(A)$. Several fundamental results in classical invariant theory have been generalized to this noncommutative setting; for example, see [KKZ3, Theorem 1.1] for a version of the Shephard-Todd-Chevalley Theorem, and KKZ2, Theorem 0.2 ] for a version of Watanabe's theorem.

Our interest here is in studying AS Gorenstein fixed rings $A^{H}$ of $A$. We have chosen to study the parametrized family $A(\alpha, \beta)$ of $\mathbb{N}$-graded down-up algebras. Down-up algebras were introduced by Benkart and Roby [BR] in 1998 as a tool to study the structure of certain posets. Since then various aspects of these algebras have been studied extensively, for example, BW, KK, KMP. Noetherian graded down-up algebras form a class of AS regular algebras of global dimension three that are generated in degree 1 by two elements, with two cubic relations; these algebras are not Koszul, but they are (3)-Koszul. Their graded automorphism groups, which depend upon the parameters $\alpha$ and $\beta$, were computed in $[\mathrm{KK}$, and are sufficiently rich to provide many non-trivial examples (e.g. in two cases the automorphism group is the entire group $\left.G L_{2}(k)\right)$.

To accomplish our study of down-up algebras, we first study AS Gorenstein fixed subrings of AS regular algebras of global dimension two. In Section 2 we prove the following extension of a commutative result [St2, Proposition 9.4] by essentially classifying all AS Gorenstein fixed subrings of AS regular algebras of dimension two.

Theorem 0.1. Let $A$ be a noetherian $A S$ regular algebra of global dimension two that is generated in degree one. Let $H$ be a finite subgroup of $\operatorname{Aut}(A)$. Then the following are equivalent.

(1) $A^{H}$ is AS Gorenstein.

2010 Mathematics Subject Classification. 16W22, 16E65, 16S38.

Key words and phrases. Artin-Schelter regular algebra, Gorenstein algebra, complete intersection, group action, trace, Hilbert series, fixed subring. 
(2) $A^{H}$ is isomorphic to $C /(\Omega)$, where $C$ is a noetherian $A S$ regular algebra of global dimension three, and $\Omega$ is a regular normal element of $C$. (Such an algebra $C /(\Omega)$ is called a hypersurface.)

For down-up algebras, two fundamental results of classical invariant theory, the Shephard-Todd-Chevalley Theorem and Watanabe's Theorem, hold in the following way.

Proposition 0.2. Let $A$ be a noetherian graded down-up algebra and let $\{1\} \neq H$ be a finite subgroup of $\operatorname{Aut}(A)$.

(1) [KKZ1, Proposition 6.4] A has no quasi-reflections of finite order. As a consequence, $A^{H}$ is not $A S$ regular, and all finite groups of graded automorphisms are "small".

(2) [KKZ2, Corollary 4.11] $A^{H}$ is AS Gorenstein if and only if the homological determinant of the $H$-action is trivial.

In studying AS Gorenstein invariant subrings we are interested in a noncommutative version of the classical result of Kac-Watanabe KW and Gordeev G1 that states that, if the fixed subring $k\left[x_{1}, \cdots, x_{n}\right]^{H}$ (for any finite subgroup $H \subset$ $\left.G L_{n}(k)\right)$ is a complete intersection, then $H$ is generated by bireflections (i.e., elements $g \in G L_{n}(k)$ such that $\left.\operatorname{rank}(g-I) \leq 2\right)$. In Section 5 we will provide suitable definitions of complete intersection and bireflection in the noncommutative setting. In dimension 2, by Theorem 0.1 all AS Gorenstein invariant subrings are hypersurfaces, and all automorphisms of finite order are trivially bireflections, and hence the first interesting case of the Kac-Watanabe/Gordeev theorem is in dimension 3 , so that it is natural to investigate generalizations of this theorem for down-up algebras. We prove the following version of Kac-Watanabe/Gordeev theorem for noetherian graded down-up algebras.

Theorem 0.3. Let $A$ be a connected graded noetherian down-up algebra and $H$ be a finite subgroup of $\operatorname{Aut}(A)$. Then the following are equivalent.

(C1) $A^{H}$ is a complete intersection (of type GK).

(C2) $A^{H}$ is cyclotomic Gorenstein and $H$ is generated by bireflections.

(C3) $A^{H}$ is cyclotomic Gorenstein.

In general, it would be interesting to understand the connection between three very different concepts

"complete intersection", "cyclotomic Gorenstein" and "bireflection"

for a general noncommutative AS regular algebra. It was proved in KKZ4 that condition (C1) implies condition (C3) for $A^{H}$, where $A$ is any noetherian AS regular algebra, so it is a natural question to ask if condition $(\mathrm{C} 1)$ is equivalent to $(\mathrm{C} 3)$ for algebras of the form $A^{H}$, where $A$ is a noetherian AS regular algebra. In [St1] R. Stanley asked when a commutative cyclotomic Gorenstein ring is a complete intersection, and he gave an example that shows that this is not always the case [St1. Example 3.9]. The answer to Stanley's question is not yet well understood, even for cyclic groups, even when $A$ is a commutative polynomial ring (see [G3]). If $A$ is a commutative polynomial ring, then condition (C1) implies (C2) by KacWatanabe/Gordeev KW, G1]. Another natural question, which is a motivating question for this paper, is whether the Kac-Watanabe/Gordeev theorem holds for general noncommutative AS regular algebras. In some cases, the fixed subrings that 
appear in Theorem 0.3 are presented explicitly as (classical) complete intersections [Examples 8.2 and 8.3 .

To prove Theorem 0.3 , we first find the finite groups $H$ that act on some graded down-up algebra $A:=A(\alpha, \beta)$ with trivial homological determinant; the set of all graded automorphisms with trivial homological determinant is denoted by $S L A(A)$, as they can be considered as analogs of the elements in the special linear group $S L_{n}(k)$. In fact, for two families of down-up algebras all finite subgroups $S L_{2}(k)$ appear in the classification, but as the "homological determinant of $g$ " for $g$ a graded automorphism of a down-up algebra is $(\operatorname{det} g)^{2}$, there are additional subgroups of $G L_{2}(k)$ in $S L A(A)$. For all values of $\alpha$ and $\beta$, there are diagonal automorphisms of $A$ of the form

$$
g=\left(\begin{array}{ll}
a & 0 \\
0 & b
\end{array}\right)
$$

and we let $O:=k^{\times} \times k^{\times}$denote the group of all elements of that form, where $k^{\times}$denotes $k \backslash\{0\}$. For some values of the parameters, these are the only kinds of graded automorphisms of $A(\alpha, \beta)$. For the parameterized family $A=A(\alpha,-1)$, there are groups of automorphisms of $A$ whose elements are either in $O$, or are of the form

$$
g=\left(\begin{array}{ll}
0 & a \\
b & 0
\end{array}\right)
$$

and we let $U$ denote the group of all elements that are of the form above, or in $O$. We show that the families of finite subgroups of $O$ and $U$ that occur in $S L A(A)$, arise as involutions of type ADE singularities of $k[x, y]$. These families of finite groups are classified in Section 3, and the eight families $Q_{i}, i=1, \ldots, 8$, that arise appear in Table 3 at the end of that section. In Section 4 we find the corresponding finite subgroups of $O$ for $k_{q}[x, y]$.

Our goal is to determine which of the AS Gorenstein subrings $A^{H}$ are "complete intersections", but even for groups of small order it is difficult to obtain an explicit presentation of $A^{H}$. Hence, an important ingredient in the proof of Theorem 0.3 is lifting the various properties in (C1-C3) from the associated graded $\operatorname{ring} \operatorname{gr}_{F} A$ to $A$, where $F$ is a filtration of $A$ (definitions are given in Section 6). For down-up algebras we often are able to choose a filtration so that $\operatorname{gr}_{F} A$ is an algebra where the computation is much easier. The following results are proved in Section 6 .

Proposition 0.4. Let $A$ be a connected graded $A S$ regular algebra and $H$ a finite subgroup of $\operatorname{Aut}(A)$. Suppose $F$ is a graded $H$-stable filtration of $A$ such that the associated Rees ring is noetherian and that the associated graded algebra $\operatorname{gr}_{F} A$ is an AS Cohen-Macaulay algebra. Let $\widehat{H}$ be the subgroup of $\left.\operatorname{Aut}_{\left(\mathrm{gr}_{F}\right.} A\right)$ induced from $H$. Then the following properties hold.

(1) If the fixed subring $\left(\operatorname{gr}_{F} A\right)^{\widehat{H}}$ is a complete intersection of type GK, then so is $A^{H}$.

(2) Assume $\operatorname{gr}_{F} A$ is a domain. The fixed subring $\left(\operatorname{gr}_{F} A\right)^{\widehat{H}}$ is cyclotomic Gorenstein if and only if $A^{H}$ is.

(3) The group $\widehat{H}$ is generated by bireflections if and only if $H$ is.

Properties of down-up algebras and their invariants are given in Section 7 and in Section 8 we determine which of the $A^{Q_{i}}$ are complete intersections of GK-type for some values of the parameters $\alpha$ and $\beta$ (see Table 4 in Section 8). In Lemma 7.3 we note that the AS Gorenstein and cyclotomic properties of $A^{H}$ depend on 
the eigenvalues of elements of $H$, and not on the parameters $\alpha$ and $\beta$. The proof of Theorem 0.3 concludes in Section 9, where the remaining cases, including $A(\alpha,-1)$ and $A(0,1)$ (that have non-diagonal automorphisms), are completed; the filteredgraded results allow us to consider only finite subgroups of $S L_{2}(k)$ and the groups $Q_{i}$.

Throughout, an element of a graded algebra usually means a homogeneous element. For simplicity, the base field $k$ is assumed to be algebraically closed of characteristic zero, though some definitions and basic properties do not require it. This paper is closely related to KKZ4, in which several different definitions of "noncommutative complete intersection" are proposed.

\section{Preliminaries}

Most of the notation and the terminology follows that of [JoZ, KKZ1, KKZ4]. For example, $\operatorname{Aut}(A)$ denotes the group of graded algebra automorphisms of $A$. For $g \in \operatorname{Aut}(A), \operatorname{Tr}_{A}(g, t)$ denotes the trace function of $g$ (as defined in KKZ1, p. 6335]).

We refer to $\mathrm{KL}$ for the definition of Gelfand-Kirillov dimension and its basic properties. However, for our purpose, we will use the following simpler definition.

Definition 1.1. Let $A$ be a connected graded algebra. The Gelfand-Kirillov dimension (or GK-dimension for short) of $A$ is defined to be

$$
\operatorname{GKdim} A=\limsup _{n \rightarrow \infty} \frac{\log \left(\sum_{i=0}^{n} \operatorname{dim} A_{i}\right)}{\log n} .
$$

This definition agrees with the original definition given in $\mathrm{KL}$ when $A$ is finitely generated, but may differ otherwise. Note that most of our algebras will be noetherian, hence finitely generated.

We recall the definition of AS regularity, see also [KKZ1, Definition 1.5].

Definition 1.2. Let $A$ be a connected graded algebra. We call A Artin-Schelter Gorenstein (or $A S$ Gorenstein for short) of dimension $d$ if the following conditions hold:

(a) $A$ has injective dimension $d<\infty$ on the left and on the right,

(b) $\operatorname{Ext}_{A}^{i}\left({ }_{A} k,{ }_{A} A\right)=\operatorname{Ext}_{A}^{i}\left(k_{A}, A_{A}\right)=0$ for all $i \neq d$, and

(c) $\operatorname{Ext}_{A}^{d}\left({ }_{A} k,{ }_{A} A\right) \cong \operatorname{Ext}_{A}^{d}\left(k_{A}, A_{A}\right) \cong k(l)$ for some $l$ (where $l$ is called the $A S$ index).

If, in addition,

(d) $A$ has finite global dimension, and

(e) $A$ has finite Gelfand-Kirillov dimension,

then $A$ is called Artin-Schelter regular (or AS regular for short) of dimension $d$.

The homological determinant hdet $: \operatorname{Aut}(A) \rightarrow k^{\times}$was introduced in [JoZ]. The following lemma will be used frequently.

Lemma 1.3. JoZ, Lemma 2.6] Let $A$ be AS Gorenstein of injective dimension d and let $g \in \operatorname{Aut}(A)$. Suppose $g$ is k-rational in the sense of [JoZ, Definitions 1.3] (e.g., if $A$ is $A S$ regular, or $A$ is PI). Then the trace of $g$ is of the form

$$
\operatorname{Tr}_{A}(g, t)=(-1)^{d}(\text { hdet } g)^{-1} t^{l}+\text { lower degree terms, }
$$

when we express $\operatorname{Tr}_{A}(g, t)$ as a Laurent series in $t^{-1}$. 
Remark 1.4. In this paper, one can view (E1.3.1) as a definition of homological determinant, or as a way of computing the homological determinant. Hence we will not review the original definition of the homological determinant in [JoZ], which uses local cohomology. Let $G$ be a subgroup of $\operatorname{Aut}(A)$. We say the homological determinant hdet of a $G$-action on $A$ is trivial if hdet $g=1$ for all $g \in G$.

We make the following general hypotheses for the rest of the paper:

(H1.4.1) Every finitely generated graded $A$-module is rational over $k$ in the sense of [JoZ, Definition 1.4], and

(H1.4.2) Every $g \in \operatorname{Aut}(A)$ is $k$-rational in the sense of [JoZ, Definition 1.3].

Similar assumptions were made in [KKZ2, Lemma 4.7]. By [JoZ] every finitely generated graded module over $A$ is rational over $k$ and every $g \in \operatorname{Aut}(A)$ is $k$ rational if one of the following holds:

(1) $A$ is noetherian PI;

(2) $A$ is a factor ring of a noetherian AS regular algebra;

(3) $A$ is a fixed subring of a noetherian AS regular algebra under a finite group action.

All the algebras in our main theorems satisfy the rationality conditions given in [JoZ, Definitions 1.3 and 1.4], and for this reason we can freely use results in [JoZ.

The following definition is needed in the next section.

Definition 1.5. A connected graded algebra $A$ is called a hypersurface if $A \cong$ $C /(\Omega)$ where $C$ is a noetherian AS regular algebra and $\Omega$ is a regular normal element in $C$.

The traces of some special automorphisms of a hypersurface can be easily computed using the following lemma.

Lemma 1.6. Let $C$ be a connected graded algebra with a regular normal element $\Omega \in C$. Let $g \in \operatorname{Aut}(C)$ such that $g(\Omega)=\lambda \Omega$ for some $\lambda \in k^{\times}$, and let $h$ be the induced automorphism of $g$ on $\bar{C}:=C /(\Omega)$. Then

(1)

$$
\operatorname{Tr}_{C}(g, t)=\frac{\operatorname{Tr}_{\bar{C}}(h, t)}{1-\lambda t^{\operatorname{deg} \Omega}}
$$

(2) Suppose that there is a sequence of elements $\left\{x_{1}, \cdots, x_{n}\right\}$ in $C$ such that, for each $i$,

(a) the image of $x_{i}$ in the factor ring $C /\left(x_{1}, \cdots, x_{i-1}\right)$ is a regular normal element,

(b) $C /\left(x_{1}, \cdots, x_{n}\right)=k$, and

(c) for each $i, g\left(x_{i}\right)=\lambda_{i} x_{i}$ for some $\lambda_{i} \in k^{\times}$.

Then

$$
\operatorname{Tr}_{C}(g, t)=\frac{1}{\left(1-\lambda_{1} t^{\operatorname{deg} x_{1}}\right) \cdots\left(1-\lambda_{n} t^{\operatorname{deg} x_{n}}\right)} .
$$

As a consequence, hdet $g=\prod_{i=1}^{n} \lambda_{i}$.

(3) Suppose that $C$ is as in part (2). Then

$$
\operatorname{Tr}_{\bar{C}}(h, t)=\frac{1-\lambda t^{\operatorname{deg} \Omega}}{\left(1-\lambda_{1} t^{\operatorname{deg} x_{1}}\right) \cdots\left(1-\lambda_{n} t^{\operatorname{deg} x_{n}}\right)} .
$$

As a consequence, hdet $h=\lambda^{-1} \prod_{i=1}^{n} \lambda_{i}$. 
Proof. (1) Denote the degree $n$ shift of a graded vector space $M$ by $M[n]$. Applying $g$ to the short exact sequence

$$
0 \rightarrow C[\operatorname{deg} \Omega] \stackrel{r_{\Omega}}{\longrightarrow} C \rightarrow C /(\Omega) \rightarrow 0,
$$

where $r_{\Omega}$ denotes the left $C$-module homomorphism defined by the right multiplication by $\Omega$, we obtain that $\operatorname{Tr}_{C}(g, t)-\operatorname{Tr}_{C}(g, t) \lambda t^{\operatorname{deg} \Omega}=\operatorname{Tr}_{\bar{C}}(h, t)$. The assertion follows.

(2) This follows from part (1) and induction on $n$. The second assertion follows by Lemma 1.3 .

(3) The first assertion follows from parts (1) and (2), and the second assertion follows from Lemma 1.3 .

A noncommutative version of "reflection" was introduced in KKZ1.

Definition 1.7. Let $A$ be a noetherian connected graded algebra with GKdim $A=$ $n$. An element $g \in \operatorname{Aut}(A)$ is called a quasi-reflection if the trace of $g, \operatorname{Tr}_{A}(g, t)$, is of the form

$$
\frac{p(t)}{(1-t)^{n-1} q(t)}
$$

where $p(t), q(t)$ are integral polynomials with $p(1) q(1) \neq 0$.

We often omit the prefix "quasi-" if no confusion occurs. If $A$ is AS regular then $p(t)=1$.

Let $G \subset \operatorname{Aut}(A)$. The fixed subring of $A$ under the $G$-action is defined to be

$$
A^{G}:=\{x \in A: g(x)=x \text {, for all } g \in G\} .
$$

If $G$ is generated by a single element $g$, we write $A^{g}$ for $A^{G}$.

\section{Proof of Theorem 0.1}

In this section a hypersurface means $C /(\Omega)$, where $C$ is a noetherian AS regular algebra of dimension three and $\Omega$ is a regular normal element in $C$. In this section $G$ is a finite subgroup of $\operatorname{Aut}(A)$.

Proof of Theorem 0.1. (2) $\Longrightarrow(1)$ Let $A^{G}$ be a hypersurface $C /(\Omega)$. By Rees's lemma [Le, Proposition 3.4(b)], $C /(\Omega)$ is AS Gorenstein.

$(1) \Longrightarrow(2)$ Conversely, we assume that $A^{G}$ is AS Gorenstein, where $A$ is an AS regular algebra of dimension two that is generated in degree 1 , and $G$ is a finite subgroup of $\operatorname{Aut}(A)$. We want to show that $A^{G}$ is a hypersurface.

Since $k$ is algebraically closed, every AS regular algebra $A$ of dimension two generated in degree 1 is isomorphic to either

$$
k_{q}[x, y]:=k\langle x, y\rangle /(y x-q x y), \quad \text { or } \quad k_{J}[x, y]:=k\langle x, y\rangle /\left(y x-x y-x^{2}\right)
$$

where $q \in k^{\times}$. We analyze fixed subrings $A^{G}$ in each case. Part of the analysis in this proof will be used in the proof of Theorem 0.3 in later sections.

Case 2.1: $A=k_{J}[x, y]$. It follows by an easy computation that every graded algebra automorphism $g$ of $A$ is of the form

$$
g: x \rightarrow a x, \quad y \rightarrow a y+b x
$$

for some $a \in k^{\times}$and $b \in k$. If $g$ is of finite order, then $g$ is of the form

$$
\rho_{a}: x \rightarrow a x, \quad y \rightarrow a y
$$


for some $a \in k^{\times}$. The trace of $\rho_{a}$ is

$$
\operatorname{Tr}_{A}\left(\rho_{a}, t\right)=\frac{1}{(1-a t)^{2}} .
$$

Hence $\operatorname{Aut}(A)$ does not contain any reflections [Definition 1.7. By KKZ2, Corollary 4.11], for every finite subgroup $G \subset \operatorname{Aut}(A), A^{G}$ is AS Gorenstein if and only if the homological determinant of $G$ is trivial. By Lemma 1.3. the homological determinant of $\rho_{a}$ is given by

$$
\text { hdet } \rho_{a}=a^{2} \text {. }
$$

When the homological determinant is trivial, $a^{2}=1$. This means that $a=1$ or $a=-1$. When $G$ is non-trivial, $G=\left\langle I d_{A}, \rho_{-1}\right\rangle$. The fixed subring $A^{G}$ is the second Veronese subalgebera $A^{(2)}$ which is generated by

$$
x^{2}, y^{2}, \quad x y \text {. }
$$

By Molien's theorem,

$$
H_{A^{G}}(t)=\frac{1}{2}\left(\frac{1}{(1-t)^{2}}+\frac{1}{(1+t)^{2}}\right)=\frac{1-t^{4}}{\left(1-t^{2}\right)^{3}} .
$$

Let $X:=x^{2}, Y:=y^{2}$ and $Z:=x y$ with $\operatorname{deg} X=\operatorname{deg} Y=\operatorname{deg} Z=2$. It is straightforward to check that $X, Y$ and $Z \in A^{G}$ satisfy the following relations:

$$
\begin{aligned}
Z X & =X Z+2 X^{2}, \\
Y X & =X Y+4 X Z+6 X^{2}, \\
Y Z & =(Z+2 X) Y+2 X Z, \quad \text { and, } \\
0 & =X Y+X Z-Z^{2} .
\end{aligned}
$$

The first three relations (E2.0.1 - E2.0.3 define an iterated Ore extension

$$
C:=k[X]\left[Z ; \delta_{1}\right]\left[Y ; \sigma_{2}, \delta_{2}\right],
$$

for appropriate automorphism $\sigma_{2}$ and derivations $\delta_{1}, \delta_{2}$, so that $C$ is a noetherian AS regular algebra of dimension 3 with Hilbert series:

$$
H_{C}(t)=\frac{1}{\left(1-t^{2}\right)^{3}} \text {. }
$$

Further, by a computation using (E2.0.1 $X Z-Z^{2}$ is a regular central element of $C$. Forming the factor algebra $C /(\Omega)$, we compute Hilbert series:

$$
H_{C /(\Omega)}(t)=\frac{1-t^{4}}{\left(1-t^{2}\right)^{3}}=H_{A^{G}}(t) .
$$

Therefore $A^{G} \cong C /(\Omega)$, which is a hypersurface. This completes Case 2.1.

For any noetherian AS Gorenstein algebra $A$ define

$$
\operatorname{SLA}(A)=\{g \in \operatorname{Aut}(A): \operatorname{hdet} g=1\}
$$

and

$$
\mathrm{SLA}_{-1}(A)=\{g \in \operatorname{Aut}(A): \operatorname{hdet} g=1 \text { or }-1\} \text {. }
$$

We record the analysis of $\operatorname{Aut}\left(k_{J}[x, y]\right)$ in the following proposition.

Proposition 2.1. Let $A=k_{J}[x, y]$. If $g \in \operatorname{SLA}(A)$ has finite order, then $g$ is either $I d_{A}$ or $\rho_{-1}$. If $g \in \mathrm{SLA}_{-1}(A)$ has finite order, then $g=I d_{A}, \rho_{-1}, \rho_{i}$, or $\rho_{-i}$ where $i^{2}=-1$. 
The following result [KKZ2, Theorem 0.2] will be used in the remaining cases.

Lemma 2.2. [KKZ2] Let $A$ be a skew polynomial ring $k_{p_{i j}}\left[x_{1}, \cdots, x_{n}\right]$, and let $G$ be a finite subgroup of $\operatorname{Aut}(A)$. Then $A^{G}$ is AS Gorenstein if and only if the hdet of the induced action of $G / R$ on $A^{R}$ is trivial, where $R$ is the subgroup of $G$ generated by all reflections in $G$.

Case 2.2: $A=k_{q}[x, y]$ where $q \in k^{\times}$and $q \neq \pm 1$. In this case, $\operatorname{Aut}(A)=k^{\times} \times k^{\times}$, and for any $g \in \operatorname{Aut}(A)$, there are $a, b \in k^{\times}$such that $g(x)=a x$ and $g(y)=b y$. The homological determinant of $g$ is $a b$ [Lemma 1.6(2)]. The following proposition is now clear.

Proposition 2.3. Let $A=k_{q}[x, y]$ where $q \neq \pm 1$. Then

$$
\operatorname{SLA}(A)=\left\{g \in \operatorname{Aut}(A) \mid g(x)=a x, g(y)=a^{-1} y, \text { where } a \in k^{\times}\right\}
$$

and

$$
\operatorname{SLA}_{-1}(A)=\left\{g \in \operatorname{Aut}(A) \mid g(x)=a x, g(y)= \pm a^{-1} y \text {, where } a \in k^{\times}\right\} .
$$

If $g \in \operatorname{Aut}(A)$ is of finite order, then $g(x)=a_{1} x$ and $g(y)=b_{1} y$ where $a_{1}, b_{1}$ are roots of unity. Every reflection of $k_{q}[x, y]$ for $q \neq \pm 1$ is of the form $g: x \rightarrow$ $x, y \rightarrow b_{2} y$, for some $b_{2}$, or $g: x \rightarrow a_{2} x, y \rightarrow y$, for some $a_{2}$. Let $G$ be a finite subgroup of $\operatorname{Aut}(A)$ such that $A^{G}$ is AS Gorenstein. Let $R$ be the subgroup of $G$ generated by reflections in $G$ (note that $R$ is normal in $G$ ). Then $A^{R}$ is the subring of $A$ generated by $x^{n}$ and $y^{m}$ for some $n, m>0$. The induced $G / R$-action, for any $\bar{g} \in G / R$, on $B:=A^{R}$ is of the form

$$
\bar{g}: x^{n} \rightarrow a x^{n}, \quad y^{m} \rightarrow b y^{m}
$$

for some roots of unity $a, b$. The homological determinant of $\bar{g}$ is $a b$ (by Lemma 1.6(2)). By Lemma 2.2, the homological determinant of $\bar{g}$ is 1 . Hence $b=a^{-1}$. This implies that $G / R$ is a cyclic group generated by some automorphism $\phi: x^{n} \rightarrow$ $a x^{n}, y^{m} \rightarrow a^{-1} y^{m}$, where $a$ is a primitive $w$ th root of unity for some $w \geq 1$. Thus $\left(A^{R}\right)^{G / R}$ is generated by $X:=\left(x^{n}\right)^{w}, Y:=x^{n} y^{m}$, and $Z:=\left(y^{m}\right)^{w}$. Then

$$
A^{G}=\left(A^{R}\right)^{G / R} \cong k_{p_{i j}}[X, Y, Z] /\left(X Z-q^{-n m\left(\begin{array}{c}
w \\
2
\end{array}\right)} Y^{w}\right),
$$

where $p_{12}=q^{n m w}, p_{13}=q^{n m w^{2}}$ and $p_{23}=q^{n m w}$. Note that $C:=k_{p_{i j}}[X, Y, Z]$ is noetherian and AS regular of dimension 3 and $\Omega:=X Z-q^{-n m\left(\begin{array}{c}w \\ 2\end{array}\right)} Y^{w}$ is a regular normal element of $C$. Therefore $A^{G} \cong C /(\Omega)$, which is a hypersurface.

Case 2.3: $A=k[x, y]$. Here $\operatorname{deg} x=\operatorname{deg} y=1$ by assumption. In this case $\operatorname{SLA}(A)=S L_{2}(k)$, and some subgroups of $\mathrm{SLA}_{-1}(A)$ will be discussed in the next section.

We need the following lemma.

Lemma 2.4. Let $B=k[X, Y]$ where $\operatorname{deg} X=d_{1}>0$ and $\operatorname{deg} Y=d_{2}>0$. If $G$ is a finite subgroup of $\operatorname{Aut}(B)$ with trivial homological determinant, then $B^{G}$ is a (commutative) hypersurface.

Proof. If $d_{1}=d_{2}$, the homological determinant agrees with the usual determinant. By Klein's classification of finite subgroups $G \subset S L_{2}(k)$, Kl1, Kl2] (see [Su, Chapter 3: Theorem 6.17, p. 404] for general algebraically closed field) and the explicit description of the fixed subrings $A^{G}$, every $A^{G}$ is a hypersurface. All these fixed subrings $A^{G}$ will be listed in Section 3 . 
Assume now that $d_{1}<d_{2}$. Every $g \in G$ must be of the form

$$
g: X \rightarrow a X, \quad Y \rightarrow b Y+c X^{v},
$$

where $a, b \in k^{\times}, c \in k$, and $v=d_{2} / d_{1}$ (if it is an integer), and if $d_{2} / d_{1}$ is not an integer, then $c=0$. By Lemma 1.6(2), hdet $g=a b$, and since hdet is trivial by hypothesis, $a=b^{-1}$. Hence $g: X \rightarrow b^{-1} X, \quad Y \rightarrow b Y+c X^{v}$. If $b=1$, then $c$ must be zero as $g$ is of finite order. This implies that $c$ is uniquely determined by $b$. As a consequence, if $g, h \in G$, then $g h g^{-1} h^{-1}$ is the identity, and hence $G$ is abelian, and is determined by the restriction $\left.G\right|_{k X}$. Since every finite subgroup of $k^{\times}$is cyclic, $G$ is generated by a single automorphism $\phi \neq 1$. Write $\phi$ in the form of (E2.4.1) (with $a=b^{-1}$ ).

If $b^{v+1} \neq 1, \phi\left(Y+c\left(b-b^{-v}\right)^{-1} X^{v}\right)=b\left(Y+c\left(b-b^{-v}\right)^{-1} X^{v}\right)$. Replacing $Y$ by $Y+c\left(b-b^{-v}\right)^{-1} X^{v}$, we may assume that $c=0$. Next we consider the case when $b^{v+1}=1$. By induction, $\phi^{s}(Y)=b^{s} Y+s b^{s-1} c X^{v}$ for all $s \geq 1$. So $\phi^{v+1}$ maps $X$ to $X$ and $Y$ to $Y+c(v+1) b^{-1} X^{v}$. Since $\phi^{v+1}$ has finite order, $c=0$. In both cases we may assume $c=0$ (after choosing a new generator $Y$ if necessary). When $c=0, B^{G}$ is isomorphic to $k\left[X^{w}, Y^{w}, X Y\right] \cong k\left[X^{\prime}, Y^{\prime}, Z^{\prime}\right] /\left(X^{\prime} Y^{\prime}-\left(Z^{\prime}\right)^{w}\right)$, where $w$ is the order of $b$. Hence $B^{G}$ is a hypersurface.

To complete Case 2.3, let $G$ be a finite subgroup of $\operatorname{Aut}(A)$ such that $A^{G}$ is Gorenstein. Then by the classical Shephard-Todd-Chevalley Theorem $B:=A^{R}$ is a commutative polynomial ring in two variables, $k[X, Y]$, with $\operatorname{deg} X$ and $\operatorname{deg} Y$ positive. By Lemma 2.2 the action of $G / R$ on $B$ has trivial homological determinant. By Lemma 2.4. $A^{G}=B^{G / R}$ is a hypersurface.

Case 2.4: $A=k_{-1}[x, y]$. By [KKZ3, Theorem 5.5] (also see Lemma 2.2), $A^{R}$ is isomorphic to a skew polynomial ring $k_{p}[X, Y]$ with $\operatorname{deg} X>0, \operatorname{deg} Y>0$ and $p= \pm 1$. Since $A$ has PI-degree 2, the PI-degree of $k_{p}[X, Y]$ is at most two. If the PI-degree of $A^{R}$ is 1 , then $p=1$ and $A^{R}$ is commutative, and by Lemma 2.4. $A^{G}=\left(A^{R}\right)^{G / R}$ is a hypersurface. It remains to consider the case when the PIdegree is 2 ; then $p=-1$. The next lemma will be used to complete the proof in this case.

Lemma 2.5. Let $B=k_{-1}[X, Y]$ where $\operatorname{deg} X>0$ and $\operatorname{deg} Y>0$. If $G$ is a finite subgroup of $\operatorname{Aut}(B)$ with trivial homological determinant, then $B^{G}$ is a hypersurface.

Proof. If $\operatorname{deg} X=\operatorname{deg} Y$, then, with respect to the basis $\{X, Y\}$,

$$
\operatorname{Aut}(A)=\left\{\left(\begin{array}{ll}
a & 0 \\
0 & b
\end{array}\right),\left(\begin{array}{ll}
0 & c \\
d & 0
\end{array}\right): a, b, c, d \in k^{\times}\right\}
$$

by [KKZ5, Lemma 1.12]. It follows from the classification in [CKWZ] that, if the homological determinant of $G$-action is trivial, then $G$ is either

(i) $C_{n}=\left\langle\left(\begin{array}{cc}a & 0 \\ 0 & a^{-1}\end{array}\right)\right\rangle$, where $a$ is a primitive $n$th root of unity, or

(ii) $D_{2 n}=\left\langle\left(\begin{array}{cc}a & 0 \\ 0 & a^{-1}\end{array}\right),\left(\begin{array}{ll}0 & 1 \\ 1 & 0\end{array}\right)\right\rangle$, where $a$ is a primitive $n$th root of unity.

Case (i): If $G=C_{n}$, then $B^{G}$ is generated by $X_{1}:=X^{n}, X_{2}:=Y^{n}$ and $X_{3}:=$ $X Y$. Similar to the commutative case, $B^{G}$ is isomorphic to

$$
k_{p_{i j}}\left[X_{1}, X_{2}, X_{3}\right] /\left(X_{1} X_{2}-(-1)^{\left(\begin{array}{l}
n \\
2
\end{array}\right)} X_{3}^{n}\right),
$$


where $p_{i j}$ are 1 when $n$ is even, and $p_{i j}$ are -1 when $n$ is odd. So $B^{G}$ is a hypersurface. by

Case (ii): If $G=D_{2 n}$, where $n$ is a positive even integer, then $B^{G}$ is generated

$$
X_{1}:=X^{n}+Y^{n}, X_{2}:=\left(X^{n}-Y^{n}\right) X Y, \quad \text { and } \quad X_{3}:=(X Y)^{2},
$$

and is isomorphic to $k\left[X_{1}, X_{2}, X_{3}\right] /(\Omega)$, where $\Omega:=X_{2}^{2}-X_{1}^{2} X_{3}+4(-1)^{\left(\begin{array}{l}n \\ 2\end{array}\right)} X_{3}^{\frac{n}{2}+1}$ is a regular element of $k\left[X_{1}, X_{2}, X_{3}\right]$. Therefore $B^{G}$ is a commutative hypersurface.

If $G=D_{2 n}$, where $n$ is a positive odd integer, then $B^{G}$ is generated by

$$
X_{1}:=X^{n}+Y^{n}, X_{2}:=\left(X^{n}-Y^{n}\right) X Y, \quad \text { and } \quad X_{3}:=(X Y)^{2},
$$

and $B^{G}$ is isomorphic to $C /(\Omega)$, where

$$
C=k\left\langle X_{1}, X_{2}, X_{3}\right\rangle /\left(\left[X_{3}, X_{1}\right]=\left[X_{3}, X_{2}\right]=0, X_{2} X_{1}+X_{1} X_{2}=-4(-1)^{\left(\begin{array}{l}
n \\
2
\end{array}\right)} X_{3}^{\frac{n+1}{2}}\right)
$$

and $\Omega:=X_{2}^{2}+X_{1}^{2} X_{3}$. Therefore $C$ is of the form

$$
k\left[X_{1}, X_{3}\right]\left[X_{2} ; \sigma, \delta\right]
$$

and so $C$ is noetherian, AS regular of dimension 3 , and $\Omega$ is a regular normal element of $C$. Hence $B^{G}$ is a hypersurface.

Assume now that $\operatorname{deg} X=d_{1}<d_{2}=\operatorname{deg} Y$. Every $g \in G$ is of the form (E2.4.1) and $v$ is an odd integer if $c \neq 0$ (otherwise $g(X) g(Y) \neq-g(Y) g(X)$ ). Using the argument in the proof of Lemma 2.4 one can assume that $c=0$. Then $B^{G}$ is isomorphic to $k_{p_{i j}}\left[X_{1}, X_{2}, X_{3}\right] /\left(X_{1} X_{2}-(-1)^{\left(\begin{array}{c}n \\ 2\end{array}\right)} X_{3}^{n}\right)$, where $p_{i j}$ is either 1 or -1 . (See the argument in case (i).) Therefore $B^{G}$ is a hypersurface, completing the proof of Lemma 2.5

By combining all these cases, $A^{G}=B^{G / R}$ is a hypersurface, where $B=A^{R}$. This finishes the proof of "(1) $\Longrightarrow(2)$ ", and therefore Theorem 0.1 follows.

Remark 2.6. A special case of Lemma 2.5 is when $G=D_{2 n}$ and $n=1$. Then $G$ is generated by the matrix $\left(\begin{array}{ll}0 & 1 \\ 1 & 0\end{array}\right)$. The fixed subring $B^{G}$ is generated by $X_{1}:=X+Y$ and $X_{2}:=(X-Y) X Y$, as $X_{3}:=(X Y)^{2}=-\frac{1}{4}\left(X_{1} X_{2}+X_{2} X_{1}\right)$ is generated by $X_{1}$ and $X_{2}$. In this case, $B^{G} \cong C /(\Omega)$ where $C$ is the down-up algebra $A(0,1)$

$$
C:=k\left\langle X_{1}, X_{2}\right\rangle /\left(X_{1}^{2} X_{2}-X_{2} X_{1}^{2}, X_{2}^{2} X_{1}-X_{1} X_{2}^{2}\right),
$$

and where $\Omega:=X_{2}^{2}-\frac{1}{4} X_{1}^{2}\left(X_{1} X_{2}+X_{2} X_{1}\right)$ is a regular central element in $C$.

When $G=D_{2}$ and $\operatorname{deg} X=\operatorname{deg} Y=1$, the fixed subring $B^{G}$ is generated by two elements of degrees 1 and 3 , respectively, and hence the classical Noether bound [No, $|G|=2$, on the degrees of generators of $A^{G}$, fails.

\section{Involutions ON ADE SINGULARITIES}

The action of $G L_{2}(k)$ on the vector space $k t_{1}+k t_{2}$ extends naturally to an action of $G L_{2}(k)$ on the commutative polynomial ring $k\left[t_{1}, t_{2}\right]$. The main goal of this section is to find all finite subgroups $H \subset G L_{2}(k)$ that satisfy the following exact sequence

$$
1 \rightarrow H \cap S L_{2}(k) \rightarrow H \stackrel{\text { det }}{\longrightarrow}\{ \pm 1\} \rightarrow 1
$$


and to characterize the action of $g \in H \backslash G$, where $G=H \cap S L_{2}(k)$, on the fixed subring $k\left[t_{1}, t_{2}\right]^{G}$. This analysis is needed for the proof of the main result Theorem 0.3 .

Throughout this paper we will use the following matrices, where $\epsilon$ is a root of unity, so we record them for future reference in the table below.

\begin{tabular}{|c|c|c|c|c|}
\hline $\mathbb{I}:=\left(\begin{array}{ll}1 & 0 \\
0 & 1\end{array}\right)$ & $s:=\left(\begin{array}{cc}0 & 1 \\
1 & 0\end{array}\right)$ & $s_{1}:=\left(\begin{array}{cc}0 & 1 \\
-1 & 0\end{array}\right)$ & $s_{2}:=\left(\begin{array}{cc}0 & -1 \\
1 & 0\end{array}\right)$ \\
\hline$d_{1}:=\left(\begin{array}{cc}-1 & 0 \\
0 & 1\end{array}\right)$ & $d_{2}:=\left(\begin{array}{cc}1 & 0 \\
0 & -1\end{array}\right)$ & $c_{\epsilon}:=\left(\begin{array}{cc}\epsilon & 0 \\
0 & \epsilon^{-1}\end{array}\right)$ & $c_{\epsilon,-}:=\left(\begin{array}{cc}-\epsilon & 0 \\
0 & \epsilon^{-1}\end{array}\right)$ \\
\hline
\end{tabular}

Table 1: Elements of $G L_{2}(k)$

Next we recall Klein's classification of the finite subgroups of $S L_{2}(k)$ (up to conjugation) $[\mathrm{K} 11, \mathrm{Kl} 2, \mathrm{Su}$, and the generators that will be used in our work. The generators for the type $E$ groups will not be needed in this paper.

$\left(A_{n}\right)$ The cyclic groups of order $n+1, C_{n+1}$, generated by $c_{\epsilon}$, where $\epsilon$ is a primitive $(n+1)$ st root of unity, for $n \geq 1$.

$\left(D_{n}\right)$ The binary dihedral groups $B D_{4(n-2)}$ of order $4(n-2)$ generated by $s_{1}$ and $c_{\epsilon}$, where $\epsilon$ is a primitive $2(n-2)$ nd root of unity, for $n \geq 4$.

$\left(E_{6}\right)$ The binary tetrahedral group $B T_{24}$ of order 24.

$\left(E_{7}\right)$ The binary octahedral group $B O_{48}$ of order 48.

$\left(E_{8}\right)$ The binary icosahedral group $B I_{120}$ of order 120 .

The fixed subrings $k\left[t_{1}, t_{2}\right]^{G}$, where $G$ is a finite subgroup of $S L_{2}(k)$, are described as ADE-type singularities, and are also known as du Val singularities, Kleinian singularities, simple surface singularities, or rational double points. For each finite subgroup $G \subset S L_{2}(k)$, the fixed subring $S:=k\left[t_{1}, t_{2}\right]^{G}$ is a hypersurface of the form $k[x, y, z] /(f)$, namely, a commutative polynomial algebra generated by variables $x, y, z$ modulo one relation $f$. We give the relation $f$ for each type of group [LW, Theorem 6.18]:

$\left(A_{n}\right) f=x^{2}+y^{2}+z^{n+1}$ for $n \geq 1$ with $\operatorname{deg} x=n+1, \operatorname{deg} y=n+1$ and $\operatorname{deg} z=2$;

$\left(D_{n}\right) f=x^{2}+y^{2} z+z^{n-1}$ for $n \geq 4$ with $\operatorname{deg} x=2(n-1), \operatorname{deg} y=2(n-2)$ and $\operatorname{deg} z=4$.

(E) $f=x^{2}+y^{3}+z^{4}$ with $\operatorname{deg} x=12, \operatorname{deg} y=8$ and $\operatorname{deg} z=6$.

$\left(E_{7}\right) f=x^{2}+y^{3}+y z^{3}$ with $\operatorname{deg} x=18, \operatorname{deg} y=12$ and $\operatorname{deg} z=8$.

(E) $f=x^{2}+y^{3}+z^{5}$ with $\operatorname{deg} x=30, \operatorname{deg} y=20$ and $\operatorname{deg} z=12$.

The first step in determining the action of $g \in H \backslash G$ is to compute its homological determinant on $k\left[t_{1}, t_{2}\right]^{G}$.

Lemma 3.1. Suppose $H$ is a finite subgroup that satisfies (E3.0.1). Let $g \in H \backslash G$, where $G=H \cap S L_{2}(k)$. Then the homological determinant of $g$ on $k\left[t_{1}, t_{2}\right]^{G}$ is -1 .

Proof. Let $A=k\left[t_{1}, t_{2}\right]$. Since $G \subset S L_{2}(k), S:=A^{G}$ is AS Gorenstein [JoZ, Theorem 3.3] and the AS index of $S$ is equal to the AS index of $A$ [CKWZ, Lemma $2.6(\mathrm{~b})]$. 
It follows from E3.0.1 that the induced action of $g$ on $S$ is an involution. Since the homological determinant is a homomorphism, hdet $\left.\right|_{S} g$ is either 1 or -1 . We will show that it is not 1 by contradiction, and so we assume that hdet $\left.\right|_{S} g=1$. Then $S^{g}$ is AS Gorenstein [JoZ, Theorem 3.3] and the AS index of $S^{g}$ is equal to the AS index of $S$ by CKWZ, Lemma 2.6(b)]. Thus the AS index of $A^{H}\left(=S^{g}\right)$ is equal to the AS index of $A$. It follows from [CKWZ, Lemma 2.6(b)] that $\operatorname{det} \phi=1$ for all $\phi \in H$, a contradiction. Therefore hdet $\left.\right|_{S} g=-1$.

In our analysis of the invariants $A^{H}:=k\left[t_{1}, t_{2}\right]^{H}$, we will consider $A^{H}=$ $\left(A^{G}\right)^{h}:=S^{h}$, where $G:=H \cap S L_{2}(k), S:=A^{G}$, and $h$ is the automorphism of $S$ induced by $g \in H \backslash G$. Hence it suffices to consider $h$ acting on the algebras $S:=k[x, y, z] /(f)$ listed as above. By Lemma 3.1 we need to describe only involutions of $S$ with negative homological determinant. We now consider each of these cases; for most of the cases we will not give an explicit description of the group $H$, as it will not be needed in later computation.

Case $A_{n}$ : For the case where $H \cap S L_{2}(k)$ is of type $A_{n}$, in Lemma 3.2 we compute the groups $H$ and determine that there are five cases. Then in Lemma 3.3 we compute, for each case, the fixed subring under $G$ (which is a hypersurface) and give a description of the automorphism $h$.

Lemma 3.2. If $H$ is a finite subgroup of $G L_{2}(k)$ satisfying (E3.0.1) such that $G:=H \cap S L_{2}(k)$ is cyclic, then, up to conjugation, $H$ is one of the following groups.

$\left(A_{n, 1}\right) H=\left\{\mathbb{I}, d_{1}\right\}=C_{2}$.

$\left(A_{n, 2}\right) H$ is generated by $d_{1}$ and $c_{\epsilon}$, where $\epsilon$ is a primitive 2 nth root of unity, and $H=C_{2 n} \times C_{2}$.

$\left(A_{n, 3}\right) H$ is generated by $d_{1}$ and $c_{\epsilon}$, where $\epsilon$ is a primitive $n$th root of unity and $n$ is an odd integer. In this case $H=C_{n} \times C_{2} \cong C_{2 n}$.

$\left(A_{n, 4}\right) H$ is generated by $c_{\epsilon,-}$, where $\epsilon$ is a primitive 4 th root of unity, and $H=$ $C_{4 n}$. Note that $H$ does not contain any (nontrivial) reflection of the vector space $k t_{1}+k t_{2}$.

$\left(A_{n, 5}\right) H$ is generated by $s$ and $c_{\epsilon}$, where $\epsilon$ is a primitive $n$th root of unity, and $H$ is the dihedral group $D_{2 n}$ of order $2 n$.

Note that, except for case $\left(A_{n, 4}\right), H$ contains either $d_{1}$ or $s$, which are classical reflections of $k t_{1}+k t_{2}$.

Proof. Let $g$ be any element in $H \backslash G$.

If $G$ is trivial, then $g^{2}=\mathbb{I}$. Since $\operatorname{det} g=-1$ [Lemma 3.1], and $g=d_{1}$ up to conjugation. This is case $\left(A_{n, 1}\right)$.

If $G$ has order 2 , then $G=\{\mathbb{I},-\mathbb{I}\}$. In this case $g$ has order 2 or 4 . If $g$ has order 2 , then $H$ is as in case $\left(A_{n, 2}\right)$ for $n=1$. If $g$ has order 4 , then $g^{2}=-\mathbb{I}$, so $g=c_{\epsilon,-}$, where $\epsilon$ is either $i$ or $-i$. This is case $\left(A_{n, 4}\right)$ for $n=1$.

Now assume that $G$ has order $n>2$. Up to conjugation, $G$ is generated by $c_{\epsilon}$, for $\epsilon$ a primitive $n$th root of unity. Write $g$ as $\left(\begin{array}{ll}a & b \\ c & d\end{array}\right)$. Since $g c_{\epsilon} g^{-1}=c_{\epsilon}^{w}$ for some $w$, a matrix computation shows that either $a=d=0$ or $b=c=0$. If $b=c=0$, $g=\left(\begin{array}{cc}-a & 0 \\ 0 & a^{-1}\end{array}\right)$ for some root of unity $a$. Since $g^{2} \in G$, the order of $a$ divides $2 n$. If the order of $a$ divides $n$, then $\left(\begin{array}{cc}a & 0 \\ 0 & a^{-1}\end{array}\right)$ is in $G$. So we can choose $g=d_{1}$, which 
is case $\left(A_{n, 2}\right)$ or $\left(A_{n, 3}\right)$. Suppose next that $a$ has order $2 k$, where $k$ divides $n$ but $2 k$ does not divide $n$, so we can write $2 k=2^{m+1} k^{\prime}$ with $k^{\prime}$ not divisible by 2 , and $n=2^{m} \ell$ for $\ell$ not divisible by 2 . Then the order of $g^{k^{\prime}} c_{\epsilon}^{2^{m}}$ is $2 n$, so we can assume $a$ has order $2 n$. Finally, if $a$ has order $2 n$, then $H$ is generated by $g$. This is either case $\left(A_{n, 4}\right)$ or case $\left(A_{n, 3}\right)$, depending upon the parity of $n$.

If $a=d=0$, up to conjugation we have $g=s$. This is case $\left(A_{n, 5}\right)$.

Let $S$ be the fixed subring $k\left[t_{1}, t_{2}\right]^{G}$, where $G$ is as in Lemma 3.2. Then (except in case $\left(A_{n, 1}\right)$ where $\left.G=\{\mathbb{I}\}\right)$, we have $S$ is isomorphic to $k\left[X_{1}, X_{2}, X_{3}\right] /\left(X_{1} X_{2}-X_{3}^{m}\right)$, where $m$ is the order of the cyclic group $G$ (in Lemma 3.2 cases $\left(A_{n, 3}\right)$ and $\left(A_{n, 5}\right)$ we have $G=C_{n}$, while in cases $\left(A_{n, 2}\right)$ and $\left(A_{n, 4}\right)$ we have $\left.G=C_{2 n}\right)$. Let $h$ be the graded algebra automorphism of $S$ induced by $g \in H \backslash G$. We next describe $h$ in each of the five cases $\left(A_{n, 1}\right)-\left(A_{n, 5}\right)$ of Lemma 3.2, and we summarize the results in Table 2 below.

$\left(A_{n, 1}\right)$ : Here $G=\{\mathbb{I}\}$, so $S=k\left[t_{1}, t_{2}\right]$ and $h: t_{1} \rightarrow-t_{1}, t_{2} \rightarrow t_{2}$. The fixed subring $S^{h}$ is $k\left[t_{1}^{2}, t_{2}\right]$.

To obtain the hypersurface $f$ given in the classification of Kleinian singularities above Lemma 3.1 in some of the cases we will change variables, and set

$$
x:=\frac{1}{\sqrt{2}}\left(X_{1}+X_{2}\right), \quad y:=\frac{1}{\sqrt{2}}\left(X_{1}-X_{2}\right) \text { and } z:=\xi X_{3},
$$

where $\xi^{n}=-1\left(\right.$ or $\left.\xi^{2 n}=-1\right)$.

$\left(A_{n, 2}\right)$ : Let $X_{1}:=t_{1}^{2 n}, X_{2}:=t_{2}^{2 n}$ and $X_{3}:=t_{1} t_{2}$ then

$S=k\left[X_{1}, X_{2}, X_{3}\right] /\left(X_{1} X_{2}-X_{3}^{2 n}\right)$ and

$$
h: X_{1} \rightarrow X_{1}, \quad X_{2} \rightarrow X_{2}, \quad X_{3} \rightarrow-X_{3} .
$$

Using the new variables $x, y, z$, we have $S=k[x, y, z] /\left(x^{2}+y^{2}+z^{2 n}\right)$ and

$$
h: x \rightarrow x, \quad y \rightarrow y, \quad z \rightarrow-z .
$$

In this case we have the same relation $f$ given for the Kleinian singularity for $C_{2 n}$. Note that $h$ lifts to a reflection of $k[x, y, z]$. The fixed subring $S^{h}$ is a subring generated by $x, y$, and $z^{2}$, which is isomorphic to a hypersurface $k\left[x, y, z^{\prime}\right] /\left(x^{2}+y^{2}+\left(z^{\prime}\right)^{n}\right)$.

$\left(A_{n, 3}\right):$ Let $X_{1}:=t_{1}^{n}, X_{2}:=t_{2}^{n}$ and $X_{3}:=t_{1} t_{2}$, then $S=k\left[X_{1}, X_{2}, X_{3}\right] /\left(X_{1} X_{2}-\right.$ $X_{3}^{n}$ ), where $n$ is odd, and

$$
h: X_{1} \rightarrow-X_{1}, \quad X_{2} \rightarrow X_{2}, \quad X_{3} \rightarrow-X_{3} .
$$

In this case, $h$ does not lift to a reflection of $k\left[X_{1}, X_{2}, X_{3}\right]$, and $h$ does not preserve the relation $f$ given in the description of the Kleinian singularities above.

$\left(A_{n, 4}\right)$ : Let $X_{1}:=t_{1}^{2 n}, X_{2}:=t_{2}^{2 n}$ and $X_{3}:=t_{1} t_{2}$, then

$S=k\left[X_{1}, X_{2}, X_{3}\right] /\left(X_{1} X_{2}-X_{3}^{2 n}\right)$ and

$$
h: X_{1} \rightarrow-X_{1}, \quad X_{2} \rightarrow-X_{2}, \quad X_{3} \rightarrow-X_{3} .
$$

Using the new variables, $S=k[x, y, z] /\left(x^{2}+y^{2}+z^{2 n}\right)$ and

$$
h: x \rightarrow-x, \quad y \rightarrow-y, \quad z \rightarrow-z .
$$

In this case, we have the same relation $f$ as in the description of the Kleinian singularities, but $h$ does not lift to a reflection of $k[x, y, z]$. 
$\left(A_{n, 5}\right):$ Let $X_{1}:=t_{1}^{n}, X_{2}:=t_{2}^{n}$ and $X_{3}:=t_{1} t_{2}$ and $S=k\left[X_{1}, X_{2}, X_{3}\right] /\left(X_{1} X_{2}-X_{3}^{n}\right)$ and

$$
h: X_{1} \rightarrow X_{2}, \quad X_{2} \rightarrow X_{1}, \quad X_{3} \rightarrow X_{3} .
$$

Using the new variables, $S=k[x, y, z] /\left(x^{2}+y^{2}+z^{n}\right)$ and

$$
h: x \rightarrow x, \quad y \rightarrow-y, \quad z \rightarrow z .
$$

In this case, $h$ lifts to a reflection of $k[x, y, z]$, and we have the same relation $f$ as in the description of the Kleinian singularities.

This completes the analysis in Case $A_{n}$, and we summarize it in Table 2 below.

\begin{tabular}{|c|c|c|c|}
\hline Case & $H$ & $S=A^{G}$ & $h$ \\
\hline$A_{n, 1}$ & $\left\langle d_{1}\right\rangle$ & $k\left[t_{1}, t_{2}\right]$ & $t_{1} \rightarrow t_{1}, t_{2} \rightarrow-t_{2}$ \\
\hline$A_{n, 2}$ & $\left\langle d_{1}, c_{\epsilon_{2 n}}\right\rangle$ & $k[x, y, z] /\left(x^{2}+y^{2}+z^{2 n}\right)$ & $x \rightarrow x, y \rightarrow y, z \rightarrow-z$ \\
\hline & & & $X_{1} \rightarrow-X_{1}, X_{2} \rightarrow X_{2}$, \\
$A_{n, 3}$ & $\left\langle d_{1}, c_{\epsilon_{n}}\right\rangle, n$ odd & $k\left[X_{1}, X_{2}, X_{3}\right] /\left(X_{1} X_{2}-X_{3}^{n}\right)$ & $X_{3} \rightarrow-X_{3}$ \\
\hline$A_{n, 4}$ & $\left\langle d_{1}, c_{\epsilon_{n n},-}\right\rangle$ & $k[x, y, z] /\left(x^{2}+y^{2}+z^{2 n}\right)$ & $x \rightarrow-x, y \rightarrow-y, z \rightarrow-z$ \\
\hline$A_{n, 5}$ & $\left\langle s, c_{\epsilon_{n}}\right\rangle$ & $k[x, y, z] /\left(x^{2}+y^{2}+z^{n}\right)$ & $x \rightarrow x, y \rightarrow-y, z \rightarrow z$ \\
\hline
\end{tabular}

Table 2: $H \cap S L_{2}(k)$ is of type $A_{n}$ (cyclic)

For types $D$ and $E$, we describe $h \in \operatorname{Aut}(S)$ without calculating $H$, since that is all the information about $H$ that is needed for Theorem 0.3 . We continue to assume $H$ satisfies (E3.0.1) and set $G=H \cap S L_{2}(k)$; let $g \in H \backslash G$ and $h \in \operatorname{Aut}(S)$ be the automorphism of the fixed subring $S=k\left[t_{1}, t_{2}\right]^{G}$ that is induced by $g$. Note that $h$ is an involution of $S$.

Case $D_{n}$ : Suppose $G=B D_{4 n}$ for $n \geq 2$. Assume $h \in \operatorname{Aut}(S)$. We first consider the case of an even integer $n \geq 4$, and we write $n=2 m$ for some $m \geq 2$. Then since $\operatorname{deg} z<\min \{\operatorname{deg} x, \operatorname{deg} y\}, h(z)=a z$ for some $a \in k^{\times}$. Since the degree of $x$ is $2(2 m-1)$, which is not a multiple of $\operatorname{deg} z$ and $\operatorname{deg} y$, we have $h(x)=b x$ for some $b \in k^{\times}$. Finally

$$
h(y)=c y+d z^{m-1}
$$

for some $c \in k^{\times}$and $d \in k$. Assume that $h$ is an involution. Then $a^{2}=b^{2}=c^{2}=1$. Since $h$ maps the relation to a scalar multiple of the relation, we have $a=1$ and $d=0$. If $n$ is an odd integer $n \geq 3$, a similar argument shows that any involution $h$ of $S$ is of the form

$$
h: x \rightarrow b x, \quad y \rightarrow c y, \quad z \rightarrow z,
$$

where $b^{2}=c^{2}=1$. Therefore we have three subcases to consider:

$\left(D_{n, 0}\right): c=b=-1$. In this case, the homological determinant of $h$ is 1 , so this case does not need to be considered.

$\left(D_{n, 1}\right): c=-1$ and $b=1$. In this case, the homological determinant of $h$ is -1 . Further, $h$ lifts to a reflection of $k[x, y, z]$ and preserves the relation $f$.

$\left(D_{n, 2}\right): c=1$ and $b=-1$. In this case, the homological determinant of $h$ is -1 .

Further, $h$ lifts to a reflection of $k[x, y, z]$ and preserves the relation $f$.

It remains to consider the case when $G=B D_{8}$, the quaternion group of order 8. In this case since $\operatorname{deg}(y)=\operatorname{deg}(z)$, we have $h(x)=b x, h(y)=c y+d z$ and $h(z)=e y+a z$. If $h$ is an involution that maps $f$ to a scalar multiple of $f$ and has homological determinant -1 , there are the following additional cases to consider: 
$\left(D_{2,3}\right):$ In this case

$$
h: x \rightarrow x, \quad y \rightarrow y / 2-3 z i / 2, \quad z \rightarrow y i / 2-z / 2 .
$$

Hence the homological determinant of $h$ is $-1, h$ lifts to a reflection of $k[x, y, z]$, and $h$ preserves the relation $f$.

$\left(D_{2,4}\right)$ : In this case

$$
h: x \rightarrow x, \quad y \rightarrow y / 2+3 z i / 2, \quad z \rightarrow-y i / 2-z / 2 .
$$

Hence the homological determinant of $h$ is $-1, h$ lifts to a reflection of $k[x, y, z]$, and $h$ preserves the relation $f$.

In cases $D_{2,3}$ and $D_{2,4}$ we show that there is no corresponding group $G$ that satisfies (E3.0.1) since one can take $x=t_{1}^{5} t_{2}-t_{1} t_{2}^{5}, y=\alpha\left(t_{1}^{4}+t_{2}^{4}\right)$ and $z=\beta t_{1}^{2} t_{2}^{2}$ for appropriate constants $\alpha$ and $\beta$ (so that $x^{2}+y^{2} z+z^{3}=0$ ) and show that there is no automorphism $g$ of $k\left[t_{1}, t_{2}\right]$ that induces the $h$ of cases $D_{2,3}$ or $D_{2,4}$ on $S=k[x, y, z] /\left(x^{2}+y^{2} z+z^{3}\right)$. One first shows that if such a $g$ exists it must be of the form $\left(\begin{array}{ll}a & 0 \\ 0 & b\end{array}\right)$ or $\left(\begin{array}{ll}0 & c \\ d & 0\end{array}\right)$, and then one shows that no such $g$ induces $h$.

Case $E_{6}$ : Similar to the case of $D_{n}$, every nontrivial graded algebra automorphism $h$ of $S$ is of the form

$$
h: x \rightarrow c x+d y^{2}, \quad y \rightarrow b y, \quad z \rightarrow a z
$$

for some $a, b, c \in k^{\times}$. If $h$ is an involution, then $a^{2}=b^{2}=c^{2}=1$ and $d=0$. The relation of $E_{6}$ implies that $b=1$. Therefore we have possibly three cases to consider:

$\left(E_{6,0}\right): c=a=-1$. In this case, the homological determinant of $h$ is 1 .

$\left(E_{6,1}\right): c=-1$ and $a=1$. In this case, the homological determinant of $h$ is -1 . Further, $h$ lifts to a reflection of $k[x, y, z]$ and preserves the relation $f$.

$\left(E_{6,2}\right): c=1$ and $a=-1$. In this case, the homological determinant of $h$ is -1 . Further, $h$ lifts to a reflection of $k[x, y, z]$ and preserves the relation $f$.

Case $E_{7}$ : Similar to the case of $E_{6}$, every nontrivial graded algebra automorphism $h$ of $S$ is of the form

$$
h: x \rightarrow c x, \quad y \rightarrow b y, \quad z \rightarrow a z
$$

for some $a, b, c \in k^{\times}$. If $h$ is an involution, then $a^{2}=b^{2}=c^{2}=1$. The relation of $E_{7}$ implies that $a=b=1$ and that $c=-1$. The homological determinant of $h$ is -1 . Further, $h$ lifts to a reflection of $k[x, y, z]$ and preserves the relation $f$.

Case $E_{8}$ : Let $h \in \operatorname{Aut}(S)$. The same argument as above shows that

$$
h: x \rightarrow c x, \quad y \rightarrow b y, \quad z \rightarrow a z
$$

for some $a, b, c \in k^{\times}$. If $h$ is an involution, then $a^{2}=b^{2}=c^{2}=1$. The relation of $E_{8}$ implies that $a=b=1$ and that $c=-1$. In this case, the homological determinant of $h$ is -1 . Further, $h$ lifts to a reflection of $k[x, y, z]$ and preserves the relation $f$.

The above analysis gives the following result.

Lemma 3.3. Suppose $H$ is a finite subgroup of $G L_{2}(k)$ that satisfies (E3.0.1), and let $G=H \cap S L_{2}(k)$. Write $S:=k\left[t_{1}, t_{2}\right]^{G}=k\left[X_{1}, X_{2}, X_{3}\right] /(f)$. Let $g \in H \backslash G$ and let $h$ be the induced involution of $g$ on $S$. Then $h$ lifts to a reflection of $k\left[X_{1}, X_{2}, X_{3}\right]$ and preserves $f$ in the following cases: 
(1) $G$ is of type $\left(A_{n, 1}\right),\left(A_{n, 2}\right)$, or $\left(A_{n, 5}\right)$.

(2) $G$ is of type $D_{n}$ for all $n \geq 4, E_{6}, E_{7}$, or $E_{8}$.

Proof. By Lemma 3.1, the homological determinant of $h$ on $S$ is -1 . So $\left(D_{n, 0}\right)$ and $\left(E_{n, 0}\right)$ cannot happen. The other cases were checked in the analysis above.

Let $O$ be the subgroup of $G L_{2}(k)$ consisting of elements of the form $\left(\begin{array}{ll}a & 0 \\ 0 & b\end{array}\right)$; then $O \cong k^{\times} \times k^{\times}$. Let $U$ be the subgroup of $G L_{2}(k)$ consisting of elements of the form $\left(\begin{array}{ll}a & 0 \\ 0 & b\end{array}\right)$ or $\left(\begin{array}{ll}0 & c \\ d & 0\end{array}\right)$. There is a surjective group homomorphism from $U$ to $\{\mathbb{I}, s\}$ defined by sending $\left(\begin{array}{ll}a & 0 \\ 0 & b\end{array}\right)$ to $\mathbb{I}$ and $\left(\begin{array}{ll}0 & c \\ d & 0\end{array}\right)$ to $s$. The kernel of this map is $O$. Hence there is a short exact sequence of groups

$$
1 \rightarrow O \rightarrow U \rightarrow\{\mathbb{I}, s\} \rightarrow 1 \text {. }
$$

It is well-known that $\operatorname{Aut}\left(k_{q}\left[t_{1}, t_{2}\right]\right)=O$ if $q \neq \pm 1$, and $\operatorname{Aut}\left(k_{-1}\left[t_{1}, t_{2}\right]\right)=U$ by KKZ5, Lemma 1.12]. The following lemma follows from the proof of Lemma 3.2 so its proof is omitted.

Lemma 3.4. Let $H$ be a finite subgroup of $O$ such that either $H$ is in $S L_{2}(k)$ or satisfies the short exact sequence (E3.0.1). Then, up to conjugation of $k t_{1}+k t_{2}$, $H$ is equal to one of the following groups.

(1) The group $C_{n}$ generated by $c_{\epsilon}$, where $\epsilon$ is a primitive $n$th root of unity. This group is denoted by $Q_{1}$.

(2) The group $C_{2 n} \times C_{2}$ generated by $d_{1}$ and $c_{\epsilon}$, where $\epsilon$ is a primitive 2 th root of unity. This group is denoted by $Q_{2}$.

(3) The group $C_{n} \times C_{2} \cong C_{2 n}$ generated by $d_{1}$ and $c_{\epsilon}$, where $\epsilon$ is a primitive $n$th root of unity and $n$ is an odd integer. This group is denoted by $Q_{3}$.

(4) The group $C_{4 n}$ generated by $c_{\epsilon,-}$, where $\epsilon$ is a primitive 4 th root of unity. This group is denoted by $Q_{4}$.

Note that $Q_{1}$ occurred in case $\left(A_{n}\right), Q_{2}$ in case $\left(A_{n, 2}\right), Q_{3}$ in case $\left(A_{n, 3}\right)$, and $Q_{4}$ in case $\left(A_{n, 4}\right)$.

Lemma 3.5. Let $H$ be a finite subgroup of $U$ that is not a subgroup of $O$. Suppose that either $\mathrm{H}$ is in $S L_{2}(k)$ or satisfies the short exact sequence (E3.0.1). Then, up to conjugation, $H$ is one of the following groups.

(1) The binary dihedral group $B D_{4 n}$, for $n \geq 1$, that is generated by $s_{1}$ and $c_{\epsilon}$, where $\epsilon$ is a primitive 2 th root of unity. This group is denoted by $Q_{5}$. Note that $B D_{4}=\left\langle s_{1}\right\rangle$ is cyclic.

(2) The dihedral group $D_{2 n}$, for $n \geq 1$, that is generated by $s$ and $c_{\epsilon}$, where $\epsilon$ is a primitive nth root of unity. This group is denoted by $Q_{6}$.

(3) The group of order $8 n$ generated by $d_{1}, s$ (or $\left.s_{1}\right)$ and $c_{\epsilon}$, where $\epsilon$ is a primitive 2 th root of unity. This group is denoted by $Q_{7}$ (it is isomorphic to $B D_{4 n} \rtimes\left(d_{1}\right)$, the semidirect product of the binary dihedral group of order $4 n$ (generated by $c_{\epsilon}$ and $s$ ) and the cyclic group of order 2 (generated by $\left.d_{1}\right)$ ).

(4) The group of order $8 n$ generated by $s$ and $c_{\epsilon,-}$, where $\epsilon$ is a primitive $4 n$th root of unity. This group is denoted by $Q_{8}$ (it is isomorphic to a semidirect product of two cyclic groups $C_{4 n} \rtimes C_{2}$, as $\left.s c_{\epsilon,-} s=c_{\epsilon,-}^{2 n-1}\right)$. 
(4') The group of order $8 n$ generated by $s_{1}$ and $c_{\epsilon,-}$, where $\epsilon$ is a primitive $4 n$th root of unity. This group is conjugate to the group in case (4), so is also denoted by $Q_{8}$.

Note that $Q_{5}$ appeared in case $\left(D_{n}\right), Q_{6}$ in case $\left(A_{n, 5}\right)$. We will see that groups $Q_{7}$ and $Q_{8}$ arise in the proof below. Although they have the same order, the groups $Q_{7}$ and $Q_{8}$ are not isomorphic (e.g. when $n=1, Q_{7}$ is the dihedral group generated by $d_{1} s$ and $s$ (here $c_{\epsilon}=\left(d_{1} s\right)^{2}$ ), while $Q_{8}$ is the direct product of two cyclic groups $\left.C_{4} \times C_{2}\right)$.

Proof of Lemma 3.5. First note that the group generated by $s_{1}$ and $Q_{4}$ is conjugate to the group generated by $s$ and $Q_{4}$ using the element

$$
\left(\begin{array}{ll}
\epsilon & 0 \\
0 & 1
\end{array}\right) \text {. }
$$

Hence the groups in case (4) and case (4') are isomorphic.

Pick $g \in H \backslash O$. By (E3.3.1), $H$ is generated by $H \cap O$ and $g$. By (E3.0.1), $\operatorname{det} g= \pm 1$. There are two cases to consider.

Case (i): Suppose there is a $g \in H \backslash O$ such that $\operatorname{det} g=1$. Then, up to conjugation, $g=s_{1}$. Since $s_{1}^{2}=-\mathbb{I},-\mathbb{I} \in H \cap O$. By Lemma 3.4 $H \cap O$ is either $Q_{1}, Q_{2}$ or $Q_{4}$. (Note that $Q_{3}$ cannot occur as $-\mathbb{I} \notin Q_{3}$.) Therefore $H$ is of cases (1), (3) or (4'), respectively.

Case (ii): Suppose $\operatorname{det} g=-1$ for all $g \in H \backslash O$. Then, up to conjugation, $g=s$. We may also assume that $s_{1} \notin H$ (otherwise this is case (i)). Since $s_{1}=s d_{1}$, $d_{1} \notin H \cap O$. By Lemma 3.4 $H \cap O$ is either $Q_{1}$ or $Q_{4}$. Therefore $H$ occurs in case (2) or (4) respectively. Finally case (4) cannot occur as $\operatorname{det}\left(s c_{\epsilon,-}\right)=1$.

We summarize the groups we have found in the table below:

\begin{tabular}{|c|c|c|}
\hline$H$ & generators & $\epsilon$ primitive root \\
\hline$Q_{1}$ & $c_{\epsilon}$ & nth root \\
$Q_{2}$ & $d_{1}, \quad c_{\epsilon}$ & 2nth root \\
$Q_{3}$ & $d_{1}, \quad c_{\epsilon}$ & odd root \\
$Q_{4}$ & $c_{\epsilon,-}$ & 4nth root \\
$Q_{5}$ & $s_{1}, \quad c_{\epsilon}$ & 2nth root \\
$Q_{6}$ & $s, \quad c_{\epsilon}$ & nth root \\
$Q_{7}$ & $d_{1}, \quad s, \quad c_{\epsilon}$ & 2nth root \\
$Q_{8}$ & $s, \quad c_{\epsilon,-}$ & 4nth root \\
\hline
\end{tabular}

Table 3: Finite subgroups of $U$ that satisfy sequence (E3.0.1)

\section{Involutions on ADE singularities, noncommutative Case}

In this section an analysis similar to that in the last section, but for the noncommutative cases that are needed later, is summarized briefly. Let $A$ be a noncommutative AS regular algebra of global dimension two that is generated in degree 1 . Let $H$ be a finite subgroup of $\operatorname{Aut}(A)$ which satisfies the following exact sequence

$$
1 \rightarrow G \rightarrow H \stackrel{\text { hdet }}{\longrightarrow}\{ \pm 1\} \rightarrow 1
$$

where $G=H \cap \operatorname{SLA}(A)$. The following lemma is similar to Lemma 3.2 (with part $\left(A_{n, 5}\right)$ not occurring) and Lemma 3.4. Its proof is omitted. 
E. KIRKMAN, J. KUZMANOVICH AND J.J. ZHANG

Lemma 4.1. Let $A=k_{q}\left[t_{1}, t_{2}\right]$ and $H$ be a finite subgroup of $\operatorname{Aut}(A)$ satisfying (E4.0.1). Suppose either

(i) $q \neq \pm 1$ (and whence $H \subset O$ ), or

(ii) $q=-1$ and $H \subset O$.

Then, up to a permutation of $\left\{t_{1}, t_{2}\right\}, H$ is equal to one of the following groups.

$\left(A_{n, 1}^{q}\right) H=\left\langle\mathbb{I}, d_{1}\right\rangle=C_{2}$.

$\left(A_{n, 2}^{q}\right) H$ is generated by $d_{1}$ and $c_{\epsilon}$, where $\epsilon$ is a primitive 2 th th root of unity, and $H=C_{2 n} \times C_{2}$.

$\left(A_{n, 3}^{q}\right) H$ is generated by $d_{1}$ and $c_{\epsilon}$, where $\epsilon$ is a primitive nth root of unity and $n$ is an odd integer. In this case, $H=C_{n} \times C_{2} \cong C_{2 n}$.

$\left(A_{n, 4}^{q}\right) H$ is generated by $c_{\epsilon,-}$, where $\epsilon$ is a primitive 4 th root of unity, and $H=$ $C_{4 n}$.

Next we compute the fixed subrings. Let $S=k_{q}\left[t_{1}, t_{2}\right]^{G}$ and let $g=d_{1} \in H \backslash G$ in the first three cases, and $g=c_{\epsilon,-} \in H \backslash G$ in case $\left(A_{n, 4}^{q}\right)$. Let $h$ be the induced automorphism of $g$ on $S$.

Lemma 4.2. Retaining the hypotheses of Lemma 4.1 and setting,

$$
S=k_{q}\left[t_{1}, t_{2}\right]^{G}=k_{p_{i j}}\left[X_{1}, X_{2}, X_{3}\right] /(f) .
$$

In cases $\left(A_{n, 1}^{q}\right)$ and $\left(A_{n, 2}^{q}\right), h$ lifts to a reflection of $k_{p_{i j}}\left[X_{1}, X_{2}, X_{3}\right]$ and preserves the relation $f$.

Proof. We consider each of the cases:

$\left(A_{n, 1}^{q}\right): S=k_{q}\left[t_{1}, t_{2}\right]$ and $h=g: t_{1} \rightarrow-t_{1}, t_{2} \rightarrow t_{2}$ is a reflection of $S$, and it lifts to $h: X_{1} \rightarrow-X_{1}, X_{2} \rightarrow X_{2}, X_{3} \rightarrow X_{3}$ and preserves $f=X_{3}$.

$\left(A_{n, 2}^{q}\right):$ Let $X_{1}:=t_{1}^{2 n}, X_{2}:=t_{2}^{2 n}$ and $X_{3}:=t_{1} t_{2}$. Then

$$
S=k_{p_{i j}}\left[X_{1}, X_{2}, X_{3}\right] /\left(X_{1} X_{2}-q^{-\left(\begin{array}{c}
2 n \\
2
\end{array}\right)} X_{3}^{2 n}\right)
$$

where $p_{12}=q^{4 n^{2}}, p_{13}=q^{2 n}$ and $p_{23}=q^{-2 n}$. It is easy to see that

$$
h: X_{1} \rightarrow X_{1}, \quad X_{2} \rightarrow X_{2}, \quad X_{3} \rightarrow-X_{3} .
$$

Note that $h$ lifts to a reflection of $k_{p_{i j}}\left[X_{1}, X_{2}, X_{3}\right]$ and preserves $f=X_{1} X_{2}-q^{-\left(\begin{array}{c}2 n \\ 2\end{array}\right)} X_{3}^{2 n}$.

$\left(A_{n, 3}^{q}\right):$ Let $X_{1}:=t_{1}^{n}, X_{2}:=t_{2}^{n}$ and $X_{3}:=t_{1} t_{2}$ then

$$
S=k_{p_{i j}}\left[X_{1}, X_{2}, X_{3}\right] /\left(X_{1} X_{2}-q^{-\left(\begin{array}{c}
n \\
2
\end{array}\right)} X_{3}^{n}\right),
$$

where $n$ is an odd integer, and where $p_{12}=q^{n^{2}}, p_{13}=q^{n}$ and $p_{23}=q^{-n}$. Then

$$
h: X_{1} \rightarrow-X_{1}, \quad X_{2} \rightarrow X_{2}, \quad X_{3} \rightarrow-X_{3} .
$$

In this case, $h$ does not lift to a reflection of $k_{p_{i j}}\left[X_{1}, X_{2}, X_{3}\right]$ and does not preserve $f=X_{1} X_{2}-q^{-\left(\begin{array}{c}n \\ 2\end{array}\right)} X_{3}^{n}$.

$\left(A_{n, 4}^{q}\right):$ Let $X_{1}:=t_{1}^{2 n}, X_{2}:=t_{2}^{2 n}$ and $X_{3}:=t_{1} t_{2}$ then

$$
S=k\left[X_{1}, X_{2}, X_{3}\right] /\left(X_{1} X_{2}-q^{-\left(\begin{array}{c}
2 n \\
2
\end{array}\right)} X_{3}^{2 n}\right),
$$

where $p_{12}=q^{4 n^{2}}, p_{13}=q^{2 n}$ and $p_{23}=q^{-2 n}$, and

$$
h: X_{1} \rightarrow-X_{1}, \quad X_{2} \rightarrow-X_{2}, \quad X_{3} \rightarrow-X_{3} .
$$


In this case, $h$ preserves $f=X_{1} X_{2}-q^{-\left(\begin{array}{c}2 n \\ 2\end{array}\right)} X_{3}^{2 n}$, but does not lift to a reflection of $k_{p_{i j}}\left[X_{1}, X_{2}, X_{3}\right]$.

\section{Definitions of Complete intersection and Bireflections}

In this section we recall the definitions of complete intersection, bireflection and cyclotomic Gorenstein in the noncommutative setting, which were proposed in KKZ4 . A set of (homogeneous) elements $\left\{\Omega_{1}, \cdots, \Omega_{n}\right\}$ in a graded algebra $C$ is called a sequence of regular normalizing elements if, for each $i$, the image of $\Omega_{i}$ in $C /\left(\Omega_{1}, \cdots, \Omega_{i-1}\right)$ is regular (i.e., a non-zero-divisor) and normal.

Definition 5.1. KKZ4 Let $A$ be a connected graded noetherian algebra.

(cci) We say $A$ is a classical complete intersection ring (or cci for short) if there is a connected graded noetherian AS regular algebra $C$ and a sequence of regular normal elements $\left\{\Omega_{1}, \cdots, \Omega_{n}\right\}$ such that $A$ is isomorphic to $C /\left(\Omega_{1}, \cdots, \Omega_{n}\right)$.

(gci) We say $A$ is a complete intersection ring of type $G K$ (or gci for short) if the Ext-algebra $\operatorname{Ext}_{A}^{*}(k, k)$ has finite GK-dimension in the sense of Definition 1.1 .

In the commutative case, these two definitions of complete intersection are equivalent, and are equivalent to the following condition, see [FHT, Theorem C] and $\mathrm{Gu}$,

(nci) the Ext-algebra $\operatorname{Ext}_{A}^{*}(k, k)$ is noetherian.

In the noncommutative case the condition (nci) is not equivalent to either (cci) or (gci), but (cci) implies (gci). We refer to KKZ4 for more details and examples.

By (E1.1.1), the GK-dimension of the Ext-algebra $\operatorname{Ext}_{A}^{*}(k, k)$ is given by

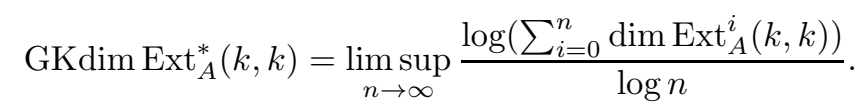

We can define a numerical invariant of the algebra $A$, called the gci number of $A$, by

$$
g c i(A)=\operatorname{GKdim} \operatorname{Ext}_{A}^{*}(k, k) .
$$

Since $A$ is noetherian, by using the minimal free resolution of the trivial $A$-module $k$, one can check that $\operatorname{Tor}_{i}^{A}(k, k)^{*} \cong \operatorname{Ext}_{A}^{i}(k, k)$ CE, Proposition 5.1, p. 120]. Thus we have

$$
g c i(A)=\limsup _{n \rightarrow \infty} \frac{\log \left(\sum_{i=0}^{n} \operatorname{dim} \operatorname{Tor}_{i}^{A}(k, k)\right)}{\log n} .
$$

A related notion is the following.

Definition 5.2. KKZ4 Let $A$ be a connected graded noetherian algebra.

(1) We say $A$ is cyclotomic if its Hilbert series $H_{A}(t)$ is of the form $p(t) / q(t)$, where $p(t)$ and $q(t)$ are coprime integral polynomials, and if all the roots of $p(t)$ are roots of unity. Note that all roots of $q(t)$ are roots of unity since $A$ is noetherian.

(2) We say $A$ is cyclotomic Gorenstein if $A$ is both cyclotomic and AS Gorenstein.

We review some results proved in [KKZ4]. 
Lemma 5.3. KKZ4 Let $A$ be a connected graded noetherian ring.

(1) If $A$ is a cci, then it is a gci.

(2) Suppose that $A$ is isomorphic to $R^{G}$ for some noetherian Auslander regular algebra $R$ and a finite group $G \subset \operatorname{Aut}(R)$. If $A$ is a gci, then it is cyclotomic Gorenstein.

The notion of cyclotomic Gorenstein is strictly weaker than the notion of complete intersection, even in the commutative case (see Stanley [St1, Example 3.9]). However, Stanley's example is not a fixed subring of an AS regular algebra.

The next two easy lemmas will be used later. Let $A=C[t ; \sigma]$ denote the skew polynomial Ore extension of $C$ by an automorphism $\sigma$ of $C$ [MR, Section 1.2.3].

Lemma 5.4. Let $C$ be a noetherian connected graded algebra, and $G$ be a finite subgroup of $\operatorname{Aut}(C)$ such that $C^{G}$ is a cci (respectively, gci).

(1) Let $\sigma \in \operatorname{Aut}(C)$ be an automorphism that commutes with all $g \in G$. By abuse of notation, let $G$ also denote the finite subgroup of $\operatorname{Aut}(C[t ; \sigma])$ induced from $G$, with $g(t)=t$ for all $g \in G$ and $\left.g\right|_{C}=g$. Then $(C[t ; \sigma])^{G}$ is a cci (respectively, gci).

(2) Let $\left\{\Omega_{1}, \cdots, \Omega_{n}\right\}$ be a sequence of regular normalizing elements in $C$ such that $g\left(\Omega_{i}\right)=\Omega_{i}$ for all $g \in G$ and all $i$. Let $B=C /\left(\Omega_{1}, \cdots, \Omega_{n}\right)$ and let $G$ also denote the subgroup of $\operatorname{Aut}(B)$ induced from $G$. Then $B^{G} \cong$ $C^{G} /\left(\Omega_{1}, \cdots, \Omega_{n}\right)$.

(3) Under the hypotheses of part (2) $B^{G}$ is a cci (respectively, gci).

Proof. (a) Since $(C[t ; \sigma])^{G}=C^{G}[t ; \sigma]$, the assertion follows.

(2) First we claim that, if $\Omega$ is a regular normal element of an algebra $D$ and $g(\Omega)=\Omega$ for all $g \in G$, then $\Omega$ is a regular normal element in $D^{G}$ and $D^{G} /(\Omega)=$ $(D / \Omega)^{G}$. To see this, we use the short exact sequence

$$
0 \rightarrow D[\operatorname{deg} \Omega] \stackrel{r_{\Omega}}{\longrightarrow} D \rightarrow D /(\Omega) \rightarrow 0 .
$$

Applying the Reynolds operator $\int:=\frac{1}{|G|} \sum_{g \in G} g$ to the sequence above, we have

$$
0 \rightarrow D^{G}[\operatorname{deg} \Omega] \stackrel{r_{\Omega}}{\longrightarrow} D^{G} \rightarrow(D /(\Omega))^{G} \rightarrow 0 .
$$

Therefore $\Omega \in D^{G}$ is a regular normal element, and $D^{G} /(\Omega)=(D / \Omega)^{G}$. The assertion follows by induction.

(3) By induction on $n$ we need to show the assertion only for $n=1$. Write $\Omega=\Omega_{1}$. Clearly $\Omega$ is a regular normal element in $C^{G}$. If $C^{G}$ is a cci (respectively, gci), so is $C^{G} /(\Omega)$. By part $(2), B^{G} \cong C^{G} /(\Omega)$, and the assertion follows.

Lemma 5.5. Let $B$ be a cci of the form $C /\left(\Omega_{1}, \cdots, \Omega_{n}\right)$, where $C$ is an $A S$ regular algebra and $\left\{\Omega_{1}, \cdots, \Omega_{n}\right\}$ is a sequence of regular normalizing elements of $C$. Suppose that $\sigma \in \operatorname{Aut}(B)$ is a graded automorphism of $B$ that lifts to a graded automorphism $\sigma^{\prime} \in \operatorname{Aut}(C)$. Let $A=B[v ; \sigma]$, and $\operatorname{deg} v=d$. Suppose that $h \in \operatorname{Aut}(A)$ is such that $h(v)=-v$ and $g:=\left.h\right|_{B}$ is a graded automorphism of $B$, where $g$ lifts to a graded automorphism $g^{\prime} \in \operatorname{Aut}(C)$. Assume that:

(1) there exists a set $\left\{x_{1}, \cdots, x_{m}\right\}$ of degree 1 elements that generate the algebra $C$, with $x_{m}$ a regular normal element in $C$;

(2) $g^{\prime} \in \operatorname{Aut}(C)$ is an involutory reflection of $C$ such that the fixed subring $C^{g^{\prime}}$ is AS regular, $g^{\prime}\left(x_{j}\right)=x_{j}$ for $j<m$, and $g^{\prime}\left(x_{m}\right)=-x_{m}$;

(3) $\sigma^{\prime}\left(x_{m}\right)=\lambda x_{m}$ for some $\lambda \in k^{\times}$, 
(4) $g^{\prime}\left(\Omega_{i}\right)=\Omega_{i}$ for all $i=1, \cdots, n$,

(5) $\sigma^{\prime}$ and $g^{\prime}$ commute.

Then the fixed subring $A^{h}$ is a cci.

Proof. By Lemma 5.4(2),

$$
\left(C /\left(\Omega_{1} \cdots, \Omega_{n}\right)\right)^{g}=C^{g^{\prime}} /\left(\Omega_{1}, \cdots, \Omega_{n}\right)
$$

where $\left\{\Omega_{1}, \cdots, \Omega_{n}\right\}$ is a sequence of regular normalizing elements in $C^{g^{\prime}}$.

By the hypotheses, $C^{g^{\prime}}$ is AS regular. Since $x_{m}$ is a normal element in $C, C^{g^{\prime}}$ is generated by $\left\{x_{1}, \cdots, x_{m-1}, x_{m}^{2}\right\}$. Formally set $x_{m}^{\prime}:=x_{m}^{2}, y_{m}:=x_{m} v \in C\left[v ; \sigma^{\prime}\right]$ and $v_{2}:=v^{2}$. Suppose that conjugation by $x_{m}$ is the graded automorphism $\sigma_{m}^{\prime}$ of $C$, i.e. $x_{m} c=\sigma^{\prime}(c) x_{m}$ for all $c \in C$. Then for any $c \in C$ we have $y_{m} c=\sigma_{m}^{\prime}\left(\sigma^{\prime}(c)\right) y_{m}, v_{2} c=\sigma^{\prime 2}(c) v_{2}$, and $v_{2} y_{m}=\lambda^{2} y_{m} v_{2}$. The map $\sigma_{m}^{\prime} \circ \sigma^{\prime}$ is an automorphism of $C^{g^{\prime}}$ since for $c \in C^{g^{\prime}}$ we have $x_{m} \sigma^{\prime}(c)=\sigma_{m}^{\prime}\left(\sigma^{\prime}(c)\right) x_{m}$, so applying $g^{\prime}$ gives $-x_{m} g^{\prime}\left(\sigma^{\prime}(c)\right)=g^{\prime}\left(\sigma_{m}^{\prime}\left(\sigma^{\prime}(c)\right)\right)\left(-x_{m}\right)$ or $x_{m} g^{\prime}\left(\sigma^{\prime}(c)\right)=g^{\prime}\left(\sigma_{m}^{\prime}\left(\sigma^{\prime}(c)\right) x_{m}\right.$, so that $x_{m}\left(\sigma^{\prime}\left(g^{\prime}(c)\right)\right)=x_{m} \sigma^{\prime}(c)=\sigma_{m}^{\prime}\left(\sigma^{\prime}(c)\right) x_{m}=g^{\prime}\left(\sigma_{m}^{\prime}\left(\sigma^{\prime}(c)\right)\right) x_{m}$, and hence $\sigma_{m}^{\prime}\left(\sigma^{\prime}(c)\right)=g^{\prime}\left(\sigma_{m}^{\prime}\left(\sigma^{\prime}(c)\right)\right)$. Let $W$ be the iterated Ore extension

$$
W:=C^{g^{\prime}}\left[Y_{m} ; \sigma_{m}^{\prime} \circ \sigma^{\prime}\right]\left[V_{2} ; \sigma^{\prime 2}\right],
$$

where we use $Y_{m}$ and $V_{2}$ as independent variables of degrees $d+\operatorname{deg} x_{m}$ and $2 d$, respectively. Then $W$ is AS regular.

We claim that $A^{h} \cong W /\left(\Omega_{1}, \ldots, \Omega_{n}, Y_{m}^{2}-\lambda x_{m}^{\prime} V_{2}\right)=: A^{\prime}$, which is a cci by definition. First, there is an algebra surjective map from $A^{\prime}$ to $A^{h}$ sending $x_{i} \rightarrow \overline{x_{i}}$ for $i<m, x_{m}^{2} \rightarrow{\overline{x_{m}}}^{2}=: \overline{x_{m}^{\prime}}, Y_{m} \rightarrow \overline{x_{m}} v=: \overline{y_{m}}$ and $V_{2} \rightarrow v^{2}=: v_{2}$, where bar indicates the image in $A$. Since $x_{m}$ is a normal element, there is a short exact sequence

$$
0 \rightarrow C\left[\operatorname{deg} x_{m}\right] \stackrel{r_{x_{m}}}{\longrightarrow} C \rightarrow C /\left(x_{m}\right) \rightarrow 0,
$$

which implies that the Hilbert series of $C$ is $H_{C}(t)=H_{C /\left(x_{m}\right)}(t)\left(1-t^{\operatorname{deg} x_{m}}\right)^{-1}$. Applying $g^{\prime}$ to (E5.5.1), it follows that the trace of $g^{\prime}$ is

$$
\operatorname{Tr}_{C}\left(g^{\prime}, t\right)=H_{C /\left(x_{m}\right)}(t) \frac{1}{1+t^{\operatorname{deg} x_{m}}} .
$$

An argument similar to the proof of Lemma 1.6 shows that

$$
\operatorname{Tr}_{A}(h, t)=H_{C /\left(x_{m}\right)}(t) \frac{\prod_{i=1}^{n}\left(1-t^{\operatorname{deg} \Omega_{i}}\right)}{\left(1+t^{\operatorname{deg} x_{m}}\right)\left(1+t^{d}\right)},
$$

where $d=\operatorname{deg} v$, and the Hilbert series of $A$ is

$$
H_{A}(t)=H_{C /\left(x_{m}\right)}(t) \frac{\prod_{i=1}^{n}\left(1-t^{\operatorname{deg} \Omega_{i}}\right)}{\left(1-t^{\operatorname{deg} x_{m}}\right)\left(1-t^{d}\right)} .
$$

By Molien's theorem, $H_{A^{h}}(t)=\left(H_{A}(t)+\operatorname{Tr}_{A}(h, t)\right) / 2$, so

$$
H_{A^{h}}(t)=H_{C /\left(x_{m}\right)}(t) \frac{\left(1+t^{d+\operatorname{deg} x_{m}}\right) \prod_{i=1}^{n}\left(1-t^{\operatorname{deg} \Omega_{i}}\right)}{\left(1-t^{2 \operatorname{deg} x_{m}}\right)\left(1-t^{2 d}\right)} .
$$


By comparing their Hilbert series, one observes that $C /\left(x_{m}\right) \cong C^{g^{\prime}} /\left(x_{m}^{2}\right)$. Then

$$
\begin{aligned}
H_{A^{h}}(t) & =H_{C /\left(x_{m}\right)}(t) \frac{\left(1+t^{d+\operatorname{deg} x_{m}}\right) \prod_{i=1}^{n}\left(1-t^{\operatorname{deg} \Omega_{i}}\right)}{\left(1-t^{2 \operatorname{deg} x_{m}}\right)\left(1-t^{2 d}\right)} \\
& =H_{C^{g^{\prime}} /\left(x_{m}^{2}\right)}(t) \frac{\left(1+t^{d+\operatorname{deg} x_{m}}\right) \prod_{i=1}^{n}\left(1-t^{\operatorname{deg} \Omega_{i}}\right)}{\left(1-t^{2 \operatorname{deg} x_{m}}\right)\left(1-t^{2 d}\right)} \\
& =H_{C^{\prime}}(t) \frac{\left(1+t^{d+\operatorname{deg} x_{m}}\right) \prod_{i=1}^{n}\left(1-t^{\operatorname{deg} \Omega_{i}}\right)}{\left(1-t^{2 d}\right)} \\
& =H_{C^{g^{\prime}}}(t) \frac{\left(1-t^{2 d+2 \operatorname{deg} x_{m}}\right) \prod_{i=1}^{n}\left(1-t^{\operatorname{deg} \Omega_{i}}\right)}{\left(1-t^{2 d}\right)\left(1-t^{d+\operatorname{deg} x_{m}}\right)} \\
& =H_{A^{\prime}}(t) .
\end{aligned}
$$

Therefore $A^{h} \cong A^{\prime}$, completing the proof.

A result of Kac-Watanabe [KW] and Gordeev [G1] states that if $G$ is a finite subgroup of $G L_{n}(k)$ acting naturally on the commutative polynomial ring $k\left[x_{1}, \cdots, x_{n}\right]$ and the fixed subring $k\left[x_{1}, \cdots, x_{n}\right]^{G}$ is a complete intersection, then $G$ is generated by bireflections (elements such that $\operatorname{rank}(g-I) \leq 2$ ). This result establishes a connection between "complete intersection" and "bireflection". We would like to extend this connection to the noncommutative case. First we need to understand what a bireflection in the noncommutative setting is.

Definition 5.6. KKZ4 Let $A$ be a noetherian connected graded algebra of GKdimension $n$. We call a graded automorphism $g$ of $A$ a bireflection if its trace has the form:

$$
\operatorname{Tr}_{A}(g, t)=\frac{p(t)}{(1-t)^{n-2} q(t)}
$$

where $p(t)$ and $q(t)$ are integral polynomials with $p(1) q(1) \neq 0$.

As in the classical case, it is convenient to view a reflection in the sense of Definition 1.7 as a bireflection, also.

Note that a bireflection is called a quasi-bireflection in KKZ4. At this point we are not able to prove a version of the Kac-Watanabe and Gordeev theorem for arbitrary AS regular algebras. In this paper we verify a version of the theorem for a class of noncommutative algebras. We make the following definitions.

Definition 5.7. Let $A$ be a noetherian connected graded algebra that is a gci. Let $\Gamma$ be a subgroup of $\operatorname{Aut}(A)$. Let $G$ be a finite subgroup of $\Gamma$ and consider the following four conditions:

(i) $A^{G}$ is a classical complete intersection.

(ii) $A^{G}$ is a complete intersection of GK type.

(iii) $A^{G}$ is cyclotomic Gorenstein and $G$ is generated by bireflections .

(iii') $A^{G}$ is cyclotomic Gorenstein.

Then

(1) We say $A$ is $\Gamma$-Gordeev if (ii) implies (iii) for every finite subgroup $G \subset \Gamma$.

(2) The algebra $A$ is said to be $\Gamma$-Kac (respectively, strongly $\Gamma$-Kac) if (iii) (respectively, (iii')) implies (ii) for every finite subgroup $G \subset \Gamma$.

(3) If (ii) and (iii) are equivalent for every finite subgroup $G \subset \Gamma$, then $A$ is called $\Gamma$-Gordeev-Kac. 
(4) If (i), (ii) and (iii) are equivalent for every finite subgroup $G \subset \Gamma$, then $A$ is called $\Gamma$-Gordeev-Kac-Watanabe (or $\Gamma-G K W$ ).

(5) Finally, if (i), (ii), (iii) and (iii') are equivalent for every finite subgroup $G \subset \Gamma$, then $A$ is called strong $\Gamma$-Gordeev-Kac-Watanabe (or strong $\Gamma$ $G K W)$.

If $\Gamma$ is the whole group $\operatorname{Aut}(A)$, then the prefix " $\Gamma-$ " is omitted.

Using the definition, the commutative polynomial ring $k\left[x_{1}, \cdots, x_{n}\right]$ is Gordeev by the Kac-Watanabe and Gordeev theorem. Theorem 0.1 implies that AS regular algebras of dimension 2 are strong GKW. Theorem 0.3 says that the down-up algebras are Gordeev-Kac.

Lemma 5.8. Let $A$ be a noetherian connected graded domain of finite $G K$-dimension, and let $G$ be a finite subgroup of $\operatorname{Aut}(A)$. Let $R$ be the subgroup of $G$ generated by bireflections in $G$. Then, for every $h \in G \backslash R$, the induced map $h^{\prime}$ of $h$ on $A^{R}$ is not a bireflection.

Proof. Since $R$ is normal in $G, h: A^{R} \rightarrow A^{R}$. By [JiZ, Lemma 5.2],

$$
\operatorname{Tr}_{A^{R}}\left(h^{\prime}, t\right)=\frac{1}{|R|} \sum_{g \in R} \operatorname{Tr}_{A}(h g, t) \text {. }
$$

Let $n=\operatorname{GK} \operatorname{dim} A$. Since $h g$ is not a bireflection, $(1-t)^{n-3} \operatorname{Tr}_{A}(h g, t)$ is analytic at $t=1$ for all $g \in R$. Hence (E5.8.1) implies that $(1-t)^{n-3} \operatorname{Tr}_{A^{R}}\left(h^{\prime}, t\right)$ is analytic at $t=1$, and therefore $h^{\prime}$ is not a bireflection of $A^{R}$.

We finish this section with an example of bireflections and noncommutative complete intersections that will be used in Section 8

Example 5.9. Let $q$ be a nonzero scalar. Let $T_{q}$ be a skew polynomial ring generated by $x_{1}, x_{2}$ and $y_{1}, y_{2}$, where $\operatorname{deg} x_{1}=\operatorname{deg} x_{2}=v>0$ and $\operatorname{deg} y_{1}=$ $\operatorname{deg} y_{2}=w>0$, subject to the relations

$$
\begin{aligned}
& x_{2} x_{1}=x_{1} x_{2}, \\
& y_{1} x_{1}=q x_{1} y_{1}, \\
& y_{1} x_{2}=q^{-1} x_{2} y_{1}, \\
& y_{2} x_{1}=q^{-1} x_{1} y_{2}, \\
& y_{2} x_{2}=q x_{2} y_{2}, \\
& y_{2} y_{1}=y_{1} y_{2} .
\end{aligned}
$$

Let $\zeta_{1}, \zeta_{2} \in \operatorname{Aut}\left(T_{q}\right)$ be defined by

$$
\zeta_{1}: x_{1} \rightarrow x_{1}, x_{2} \rightarrow x_{2}, y_{1} \rightarrow-y_{1}, y_{2} \rightarrow-y_{2},
$$

and

$$
\zeta_{2}: x_{1} \rightarrow x_{2}, x_{2} \rightarrow x_{1}, y_{1} \rightarrow c y_{2}, y_{2} \rightarrow c^{-1} y_{1}
$$

for some $c \in k^{\times}$. By choosing a new $y_{2}$, we may assume $c=1$.

Case (i): We claim that the fixed subring $T_{q}^{\zeta_{1}}$ is a cci. It is easy to see that

$$
\operatorname{Tr}_{T^{q}}\left(\zeta_{1}, t\right)=\frac{1}{\left(1-t^{v}\right)^{2}\left(1+t^{w}\right)^{2}}
$$


so $\zeta_{1}$ is a bireflection. The fixed subring $T_{q}^{\zeta_{1}}$ is isomorphic to $C /(\Theta)$, where $C$ is the skew polynomial ring generated by $x_{1}, x_{2}, Y_{1}:=y_{1}^{2}, Y_{2}:=y_{2}^{2}$ and $Y_{3}:=y_{1} y_{2}$ subject to the relations

$$
\begin{aligned}
& x_{2} x_{1}=x_{1} x_{2}, \\
& Y_{1} x_{1}=q^{2} x_{1} Y_{1}, \\
& Y_{1} x_{2}=q^{-2} x_{2} Y_{1}, \\
& Y_{2} x_{1}=q^{-2} x_{1} Y_{2}, \\
& Y_{2} x_{2}=q^{2} x_{2} Y_{2}, \\
& Y_{2} Y_{1}=Y_{1} Y_{2}, \\
& Y_{3} x_{1}=x_{1} Y_{3}, \\
& Y_{3} x_{2}=x_{2} Y_{3}, \\
& Y_{3} Y_{1}=Y_{1} Y_{3}, \\
& Y_{3} Y_{2}=Y_{2} Y_{3},
\end{aligned}
$$

and $\Theta:=Y_{1} Y_{2}-Y_{3}^{2}$. Therefore $T_{q}^{\zeta_{1}}$ is a cci. Note that $C \cong T_{q^{2}}\left[Y_{3}\right]$ as ungraded algebras.

Case (ii): Next we prove that the fixed subring $T_{q}^{\zeta_{2}}$ is a cci. Since $T_{q}$ is a "twisted tensor product" of $k\left[x_{1}, x_{2}\right]$ with $k\left[y_{1}, y_{2}\right], T_{q} \cong k\left[x_{1}, x_{2}\right] \otimes k\left[y_{1}, y_{2}\right]$ as graded vector spaces, we can compute the trace of $\zeta_{2}$ by computing the trace of the action of $\zeta_{2}$ on $k\left[x_{1}, x_{2}\right]$ and $k\left[y_{1}, y_{2}\right]$ separately. Hence,

$$
\operatorname{Tr}_{T_{q}}\left(\zeta_{2}, t\right)=\frac{1}{\left(1-t^{2 v}\right)\left(1-t^{2 w}\right)}
$$

and $\zeta_{2}$ is a bireflection of $T_{q}$. Molien's Theorem shows that the fixed subring has Hilbert series

$$
H_{T_{q}^{\zeta_{2}}}(t)=\frac{1-t^{2(v+w)}}{\left(1-t^{v}\right)\left(1-t^{w}\right)\left(1-t^{2 v}\right)\left(1-t^{2 w}\right)\left(1-t^{v+w}\right)} .
$$

If $q= \pm 1$, using $H_{T_{q}^{\zeta_{2}}}(t)$ one sees that the fixed subring $T_{q}^{\zeta_{2}}$ is isomorphic to $C /(\Theta)$, where $C$ is the skew polynomial ring generated by

$$
\begin{gathered}
X_{1}:=x_{1}+x_{2}, X_{2}:=x_{1} x_{2}, Y_{1}:=y_{1}+y_{2}, Y_{2}:=y_{1} y_{2}, \\
Z_{1}:=y_{1} x_{1}+y_{2} x_{2},
\end{gathered}
$$

and subject to the relations

$$
\begin{aligned}
X_{2} X_{1} & =X_{1} X_{2}, \\
Y_{1} X_{1} & =q X_{1} Y_{1}, \\
Y_{1} X_{2} & =X_{2} Y_{1}, \\
Y_{2} X_{1} & =X_{1} Y_{2}, \\
Y_{2} X_{2} & =X_{2} Y_{2}, \\
Y_{2} Y_{1} & =Y_{1} Y_{2}, \\
Z_{1} X_{1} & =q X_{1} Z_{1}, \\
Z_{1} X_{2} & =X_{2} Z_{1}, \\
Z_{1} Y_{1} & =q Y_{1} Z_{1}, \\
Z_{1} Y_{2} & =Y_{2} Z_{1},
\end{aligned}
$$


and

$$
\Theta:=\left(X_{1} Y_{1}-2 q Z_{1}\right)^{2}-q\left(X_{1}^{2}-2 X_{2}\right)\left(Y_{1}^{2}-2 Y_{2}\right)-8 q X_{2} Y_{2}+2 q X_{2} Y_{1}^{2}+2 q X_{1}^{2} Y_{1}
$$

is a central element of $C$. Therefore $T_{q= \pm 1}^{\zeta_{2}}$ is a cci.

If $q \neq \pm 1$, we claim that again Molien's Theorem can be used to show that the fixed subring $T_{q}^{\zeta_{2}}$ is isomorphic to $C /\left(\Theta_{1}, \Theta_{2}\right)$, where $C$ is the AS regular algebra generated by

$$
\begin{gathered}
X_{1}:=x_{1}+x_{2}, X_{2}:=x_{1} x_{2}, Y_{1}:=y_{1}+y_{2}, Y_{2}:=y_{1} y_{2}, \\
Z_{1}:=q\left(x_{1} y_{1}+x_{2} y_{2}\right)=y_{1} x_{1}+y_{2} x_{2}, \quad Z_{2}:=x_{1} y_{2}+x_{2} y_{1}=q\left(y_{2} x_{1}+y_{1} x_{2}\right),
\end{gathered}
$$

and subject to the relations

$$
\begin{aligned}
X_{2} X_{1} & =X_{1} X_{2} \\
Y_{1} X_{1} & =X_{1} Y_{1}+\left(q^{-1}-1\right)\left(Z_{2}-Z_{1}\right) \\
Y_{1} X_{2} & =X_{2} Y_{1} \\
Y_{2} X_{1} & =X_{1} Y_{2} \\
Y_{2} X_{2} & =X_{2} Y_{2} \\
Y_{2} Y_{1} & =Y_{1} Y_{2} \\
Z_{1} X_{1} & =q X_{1} Z_{1}+q\left(q^{-1}-q\right) X_{2} Y_{1}, \\
Z_{1} X_{2} & =X_{2} Z_{1} \\
Z_{1} Y_{1} & =q^{-1} Y_{1} Z_{1}+\left(q-q^{-1}\right) X_{1} Y_{2}, \\
Z_{1} Y_{2} & =Y_{2} Z_{1} \\
Z_{2} X_{1} & =q^{-1} X_{1} Z_{2}+\left(q-q^{-1}\right) X_{2} Y_{1}, \\
Z_{2} X_{2} & =X_{2} Z_{2} \\
Z_{2} Y_{1} & =q Y_{1} Z_{2}+\left(1-q^{2}\right) X_{1} Y_{2}, \\
Z_{2} Y_{2} & =Y_{2} Z_{2} \\
Z_{2} Z_{1} & =Z_{1} Z_{2}+\left(1-q^{2}\right) X_{1}^{2} Y_{2}+\left(q^{2}-1\right) X_{2} Y_{1}^{2},
\end{aligned}
$$

with a central regular sequence $\left\{\Theta_{1}, \Theta_{2}\right\}$, where

$$
\Theta_{1}:=Y_{1} X_{1}-q^{-1} X_{1} Y_{1}-\left(1-q^{-2}\right) Z_{1}
$$

and

$$
\begin{gathered}
\Theta_{2}:=Z_{2} Z_{1}-q^{-2} Z_{1} Z_{2}-\left(q^{2}-q^{-2}\right) X_{2}\left(Y_{1}^{2}-2 Y_{2}\right) \\
=\left(1-q^{-2}\right) Z_{1} Z_{2}+\left(1-q^{2}\right) X_{1} Y_{2}+\left(q^{-2}-1\right) X_{2} Y_{1}^{2}+2\left(q^{2}-q^{-2}\right) X_{2} Y_{2} .
\end{gathered}
$$

First one checks that the associated elements of $T_{q}$ satisfy the fifteen relations in $C$ and the additional relations $\Theta_{1}$ and $\Theta_{2}$. Next one checks the Diamond Lemma to see that $X_{1}, X_{2}, Y_{1}, Y_{2}, Z_{1}$ and $Z_{2}$ form a PBW basis for $C$. Then we filter $C$ with the filtration $F$, where degree $Z_{i}=5$ and degree $X_{i}=$ degree $Y_{i}=3$, so that there is an algebra surjection from $\operatorname{gr}_{F} C$ onto the skew polynomial ring $D$, where $D$ is an algebra of the form $k_{p_{i, j}}\left[w_{1}, \ldots, w_{6}\right]$, which is an AS regular domain of dimension 6 . The PBW basis for $C$ shows that there is a vector space isomorphism from $\operatorname{gr}_{F} C$ to $D$, and hence the algebra map from $\operatorname{gr}_{F} C$ to $D$ is an isomorphism. As $\operatorname{gr}_{F} C$ is an AS regular domain of dimension 6, it follows that $C$ is an AS regular domain of dimension 6 . 
It can be checked that $\Theta_{1}$ and $\Theta_{2}$ are central elements of $C$. As $C$ is a domain, $\Theta_{1}$ is a regular element of $C$. Now using the filtration $\mathcal{F}$, where degree $X_{i}=\operatorname{degree}$ $Y_{i}=$ degree $Z_{i}=1$, we see that

$$
\operatorname{gr}_{\mathcal{F}}\left(\frac{C}{\left(\Theta_{1}\right)}\right) \cong \frac{\operatorname{gr}_{\mathcal{F}} C}{\left(\operatorname{gr}_{\mathcal{F}} \Theta_{1}\right)} \cong \frac{k\left[X_{1}, Y_{1}\right]}{\left(X_{1} Y_{1}\right)}\left\langle Y_{1}, Y_{2}, Z_{1}, Z_{2}\right\rangle,
$$

is an iterated Ore extension, where $\operatorname{gr}_{\mathcal{F}} \Theta_{2}$ is a regular element of $\operatorname{gr}_{\mathcal{F}}\left(C /\left(\Theta_{1}\right)\right)$, and hence $\Theta_{2}$ is a regular element of $C /\left(\Theta_{1}\right)$. It follows that $\left\{\Theta_{1}, \Theta_{2}\right\}$ is a regular central sequence of $C$, and therefore $T_{q}^{\zeta_{2}}$ is a cci.

Case (iii): Let $G=\left\langle\zeta_{1}, \zeta_{2}\right\rangle$. We show that $T_{q}^{G}$ is a cci. Note that $G \cong C_{2} \times C_{2}$. So $T_{q}^{G}=\left(T_{q}^{\zeta_{1}}\right)^{\phi_{2}}$, where $\phi_{2}$ is the induced automorphism of $\zeta_{2}$ in $T_{q}^{\zeta_{1}}$. By case (i), $T_{q}^{\zeta_{1}} \cong\left(T_{q^{2}}\left[Y_{3}\right]\right) /(\Omega)$. Since $\phi_{2}$, as an automorphism of $T_{q^{2}}\left[Y_{3}\right]$, preserves $Y_{3}$ and $\Omega$, it follows from Lemma $5.4(1,2)$ that it suffices to show $T_{q^{2}}^{\phi_{2}}$ is a cci. Now $\phi_{2}$ is a $\zeta_{2}$ with $c$ replaced by $c^{2}$ (and $\operatorname{deg} Y_{1}=\operatorname{deg} Y_{2}=2 w$ ). By case (ii), $T_{q^{2}}^{\phi_{2}}$ is a cci, and therefore $T_{q}^{G}$ is a cci.

\section{Filtrations}

In the first part of this section we review the May spectral sequence $\mathrm{Ma}$ ] that is associated to a filtered algebra. Let $\Lambda$ be a group, usually taken to be $\mathbb{Z}^{d}$ for some integer $d$. A $\Lambda$-graded space $X$ is called $\Lambda$-locally finite if $X=\bigoplus_{g \in \Lambda} X_{g}$, where each $X_{g}$ is finite dimensional over $k$. Assume that $X:=\bigoplus_{n \in \mathbb{Z}, g \in \Lambda} X_{g}^{n}$ is a $\mathbb{Z} \times \Lambda$-graded, $\Lambda$-locally finite, complex with differential $d$ of degree $(1,0) \in \mathbb{Z} \times \Lambda$.

Definition 6.1. A filtration on the complex $X$ is a sequence of $\mathbb{Z} \times \Lambda$-graded subcomplexes of $X$, say $F:=\{F(n) \mid n \in \mathbb{Z}\}$, such that the following hold:

(1) $F(n+1) \subset F(n)$ for all $n$,

(2) $\bigcap_{n \in \mathbb{Z}} F(n)=\{0\}$, and

(3) $\bigcup_{n \in \mathbb{Z}} F(n)=X$.

The associated graded complex is defined to be

$$
\operatorname{gr}_{F} X=\bigoplus_{n \in \mathbb{Z}} F(n) / F(n+1)
$$

with the induced differential.

Later we consider filtrations only on complexes that are concentrated in degree $\{0\} \times \Lambda$; in this case, the differential is trivial. For any $a \in F(n)$, let $\widehat{a}$ denote the element $a+F(n+1)$ in $F(n) / F(n+1)$. Then $\operatorname{gr}_{F} X$ is a $\mathbb{Z}^{2} \times \Lambda$-graded $k$-space where the second $\mathbb{Z}$-grading comes from the filtration. For any $a$, the differential $d$ is defined to be

for all $\widehat{a} \in F(n) / F(n+1)$.

$$
d(\widehat{a})=\widehat{d(a)}
$$

Since $\operatorname{gr}_{F} X$ is a complex, we can compare the cohomologies $H\left(\operatorname{gr}_{F} X\right)$ with $H(X)$. Next is a standard lemma [Mc, Theorem 2.6].

Lemma 6.2. Retain the above notation. Suppose that $X$ is $\Lambda$-locally finite. Then there is a convergent spectral sequence

$$
E_{1}^{p, q}:=H^{p+q}(F(p) / F(p+1)) \Longrightarrow H^{p+q}(X) .
$$


Proof. Let $g \in \Lambda$. Restricting to a $g$-homogeneous component of $X$, we may assume that $X$ is finite dimensional, so the filtration is bounded. The assertion now follows from $[\mathrm{Mc}$, Theorem 2.6].

Let $A$ be a $\mathbb{Z} \times \Lambda$-graded algebra which is a dg (differential graded) algebra with respect to the first grading, and is a locally finite algebra with respect to the second grading.

Definition 6.3. Let $A$ be a dg algebra. An algebra filtration of $A$ is a filtration of the complex $A$ that satisfies the following extra conditions:

(1) $k \subset F(0)$,

(2) $F(1) \subset F(0)_{*, \geq 1}$,

(3) $F(n) F(m) \subset \bar{F}(n+m)$ for all $n, m \in \mathbb{Z}$.

The associated dg algebra is defined to be

$$
\operatorname{gr}_{F} A=\bigoplus_{n \in \mathbb{Z}} F(n) / F(n+1) \text {. }
$$

Next we consider a version of the May spectral sequence associated to a filtered algebra; see [Ma, Theorem 3]. We say $A$ is augmented if there is a dg algebra homomorphism $\epsilon: A \rightarrow k$. Suppose $\Lambda=\mathbb{Z}^{d}$. We say a $\Lambda$-graded vector space $X$ is connected graded if $X=\bigoplus_{g \in \mathbb{N}^{d}} X_{g}$ and $X_{0}=k$.

Proposition 6.4. Let $A$ be $a \mathbb{Z} \times \Lambda$-graded filtered $d g$ algebra that is $\Lambda$-locally finite and connected graded. Then there is a convergent spectral sequence

$$
\operatorname{Tor}_{p+q}^{\mathrm{gr} A}(k, k)(p) \Longrightarrow \operatorname{Tor}_{p+q}^{A}(k, k) \text {. }
$$

Proof. Let $I:=\operatorname{ker} \epsilon$ be the augmented ideal of $A$. Let $B(A)$ be the bar complex $(T(s I), \partial)$ where $s$ is the homological degree shift, see [Ma, Section 3]. Then $\operatorname{Tor}_{n}^{A}(k, k)$ can be computed by [Ma, (3.4)] as follows

$$
\operatorname{Tor}_{n}^{A}(k, k)=H^{n}(B(A)) \text {. }
$$

Since $A$ is filtered, so is $B(A)$ by the induced filtration on $I$. Since $A$ is $\Lambda$-locally finite and connected graded, so is $B(A)$. By Lemma 6.2 there is a convergent spectral sequence

$$
H^{p+q}(\operatorname{gr} B(A)(p)) \Longrightarrow H^{p+q}(B(A))=\operatorname{Tor}_{n}^{A}(k, k) .
$$

Since $\operatorname{gr} B(A) \cong B(\operatorname{gr} A)$, the left-most term in the above displayed equation is isomorphic to $\operatorname{Tor}_{p+q}^{\mathrm{gr} A}(k, k)(p)$, and the assertion follows.

The filtrations used in [Ma, Theorem 3] are bounded in one of the directions; see [Ma, Condition (4.1) or (4.1')]. The filtration in Proposition 6.4 is not necessarily bounded in any direction, but the algebra $A$ has an extra $\Lambda$-grading that is $\Lambda$-locally finite and connected graded. This is the only difference between [Ma, Theorem 3] and Proposition 6.4 Now we return to connected $\mathbb{Z}$-graded algebras $A$.

Lemma 6.5. Let $A$ be a filtered algebra and let $B=\operatorname{gr}_{F} A$. Suppose that

(a) $\Lambda=\mathbb{Z}$,

(b) $A$ is connected graded with respect to the $\Lambda$-grading,

(c) $F$ is a filtration of $A$ and each $F(n)$ is a $\mathbb{Z}$-graded vector space, and

(d) the Rees ring $\bigoplus_{n \in \mathbb{Z}} F(n) t^{-n}$ is noetherian.

Then the following hold. 
(1) $A$ and $B$ are noetherian.

(2) If $B$ has finite global dimension, so does $A$.

(3) If $B$ is a gci, then so is $A$.

(4) If $B$ is Gorenstein (respectively, AS Gorenstein), so is $A$.

(5) If $B$ is $A S$ regular, so is $A$.

Proof. (1) This is a graded version of [LvO, Proposition 1.2.3 in Chapter 2].

(2) This is a graded version of [LvO, Theorem 7.2.11 in Chapter 1].

(3) By (E5.1.1)

$$
\operatorname{gci}(A)=\limsup _{n \rightarrow \infty} \frac{\log \left(\sum_{i=0}^{n} \operatorname{dim} \operatorname{Tor}_{i}^{A}(k, k)\right)}{\log n} .
$$

Since $B$ is a complete intersection of GK-type, $g c i(\operatorname{gr} A)<\infty$. By Proposition 6.4 $\operatorname{gci}(A)<\operatorname{gci}(\operatorname{gr} A)<\infty$. So $A$ is a gci.

(4) This is a consequence of $[\mathrm{Bj}$, Proposition 3.1].

(5) This follows from parts (4) and (2).

For the rest of this section we assume the hypotheses of Lemma 6.5 (a,b,c,d) whenever a filtration appears. The following lemma is straightforward.

Lemma 6.6. Let $F$ be a filtration on a $\Lambda$-graded $A$, with the degree being $\operatorname{deg}_{\Lambda}$. Then the associated graded algebra $\operatorname{gr}_{F} A$ is a $\mathbb{Z} \times \Lambda$-graded algebra with $\operatorname{deg}_{\text {gr }} \widehat{a}=$ $\left(n, \operatorname{deg}_{\Lambda} a\right)$ if $a \in F(n) \backslash F(n+1)$.

Let $g \in \operatorname{Aut}(A)$. We say $g$ preserves the filtration $F$ if $g(F(n)) \subset F(n)$ for all $n$. A subgroup $G \subset \operatorname{Aut}(A)$ preserves the filtration $F$ if every $g \in G$ preserves $F$.

Lemma 6.7. Let $F$ be a filtration of $A$ with $B=\operatorname{gr}_{F} A$. Let $G$ be a subgroup of $\operatorname{Aut}(A)$ such that $G$ preserves the filtration $F$.

(1) For each $g \in G$, there is an induced automorphism $\widehat{g}: B \rightarrow B$ defined by

$$
\widehat{g}(\widehat{a})=\widehat{g(a)}
$$

for all $a \in F(n) \backslash F(n+1)$.

(2) The map $\Phi: g \rightarrow \widehat{g}$ defines a group homomorphism from $G \rightarrow \operatorname{Aut}(B)$.

(3) If $G$ is finite, then $B^{\Phi(G)}=\operatorname{gr}_{F^{\prime}} A^{G}$, where $F^{\prime}$ is the restriction of $F$ to $A^{G}$.

(4) Every non-trivial element in the kernel of $\Phi$ has infinite order.

Proof. $(1,2)$ This is clear.

(3) Since $F^{\prime}(n)=F(n) \cap A^{G}$, we have $\operatorname{gr}_{F^{\prime}} A^{g} \subset B^{\Phi(G)}$ (even if $G$ is infinite). Now assume $G$ is finite, and let $\int$ be the Reynolds operator $\frac{1}{|G|} \sum_{g \in G} g$. Since $G$ is finite and char $k=0, \int$ defines an exact functor. Starting with the short exact sequence

$$
0 \rightarrow F(n+1) \rightarrow F(n) \rightarrow F(n) / F(n+1) \rightarrow 0
$$

for each $n$, we obtain that a short exact sequence

$$
0 \rightarrow \int \cdot F(n+1) \rightarrow \int \cdot F(n) \rightarrow \int \cdot(F(n) / F(n+1)) \rightarrow 0,
$$

which is

$$
0 \rightarrow F(n+1)^{G} \rightarrow F(n)^{G} \rightarrow(F(n) / F(n+1))^{\Phi(G)} \rightarrow 0 .
$$

Since $F(n)^{G}=F(n) \cap A^{G}$, the assertion follows. 
(4) Let $g$ be an element in the kernel of $\Phi$. If $1 \neq g \in G$ has finite order, we replace $G$ by the subgroup generated by $g$. By part (3), $\operatorname{gr}_{F^{\prime}} A^{G}=\left(\operatorname{gr}_{F} A\right)^{\Phi(G)}$. Since $g \neq 1, A^{G} \neq A$. Thus $\left(\operatorname{gr}_{F} A\right)^{\Phi(G)} \neq \operatorname{gr}_{F} A$. Thus $\Phi(G) \neq\{1\}$, or $\Phi(g) \neq 1$, and hence the assertion follows.

In the next proposition, when working with the Hilbert series of $\operatorname{gr}_{F} A$ and the trace of $g \in \operatorname{Aut}\left(\operatorname{gr}_{F} A\right.$ ), only the $\Lambda$-grading (or the second grading) of $\operatorname{gr}_{F} A$ is used.

Proposition 6.8. Let $F$ be a filtration and $B=\operatorname{gr}_{F} A$. Consider $B$ as a connected graded algebra using the second grading of $B$. Let $g \in \operatorname{Aut}(A)$ be such that $g$ preserves the filtration $F$. Then the following hold.

(1) $\operatorname{Tr}_{B}(\Phi(g), t)=\operatorname{Tr}_{A}(g, t)$.

(2) $H_{B}(t)=H_{A}(t)$. As a consequence, $B$ is cyclotomic if and only if $A$ is.

(3) $g$ is a reflection if and only if $\Phi(g)$ is.

(4) $g$ is a bireflection if and only if $\Phi(g)$ is.

(5) $\operatorname{hdet} g=1$ if and only if hdet $\Phi(g)=1$.

(6) Suppose both $A$ and $B$ are $A S$ Cohen-Macaulay domains [JoZ, Definition 0.1]. Then $B$ is AS Gorenstein (respectively, cyclotomic Gorenstein) if and only if $A$ is.

Proof. (1) Again we consider the short exact sequence of graded spaces

$$
0 \rightarrow F(n+1) \rightarrow F(n) \rightarrow F(n) / F(n+1) \rightarrow 0 .
$$

Then

$$
\operatorname{Tr}_{A}\left(\left.g\right|_{F(n)}, t\right)-\operatorname{Tr}_{A}\left(\left.g\right|_{F(n+1)}, t\right)=\operatorname{Tr}_{B}\left(\left.\Phi(g)\right|_{F(n) / F(n+1)}, t\right) .
$$

Since $A$ is $\Lambda$-locally finite, so is $B$. Then, for each fixed power $t^{d}$, the coefficients of $t^{d}$ in $\operatorname{Tr}\left(\left.\Phi(g)\right|_{F(n) / F(n+1)}, t\right)$ are nonzero for only finitely many $n$. Thus

$$
\begin{aligned}
\operatorname{Tr}_{B}(\Phi(g), t) & =\sum_{n} \operatorname{Tr}_{B}\left(\left.\Phi(g)\right|_{F(n) / F(n+1)}, t\right) \\
& =\sum_{n} \operatorname{Tr}\left(\left.g\right|_{F(n)}, t\right)-\operatorname{Tr}\left(\left.g\right|_{F(n+1)}, t\right) \\
& =\operatorname{Tr}_{A}(g, t) .
\end{aligned}
$$

$(2,3,4,5)$ These are consequence of (1).

(6) By Stanley's theorem [JoZ, Theorem 6.2], the AS Gorenstein property of $A$ is determined by the Hilbert series of $A$. The assertions follow from part (2).

Now we are ready to prove Proposition 0.4 .

Proof. (1) This follows from Lemmas 6.5(3) and 6.7(3).

(2) This follows from Proposition 6.8(2,6) and Lemma 6.7(3).

(3) This follows from Proposition 6.8(4).

Proposition 6.9. Let $A$ be a filtered algebra such that $B:=\operatorname{gr}_{F} A$ is a noetherian connected graded $A S$ Gorenstein domain. Let $\Gamma$ be a subgroup of $\operatorname{Aut}(A)$, and let $\Gamma^{\prime}=\Phi(\Gamma)$.

(1) If $B$ is $\Gamma^{\prime}-K a c$, then $A$ is $\Gamma-K a c$.

(2) If $B$ is strongly $\Gamma^{\prime}-K a c$, then $A$ is strongly $\Gamma-K a c$. 
Proof. Let $G$ be a finite subgroup of $\Gamma$, and let $G^{\prime}=\Phi(G)$. The filtration $F$ on $A$ induces a filtration $F^{\prime}$ on $A^{G}$ such that $\operatorname{gr}_{F^{\prime}} A^{G}=B^{G^{\prime}}$. The hypotheses in Lemma 6.5)(a-d) hold for the new filtration $F^{\prime}$.

(1) Suppose now that $B$ is $\Gamma^{\prime}$-Kac. Assume that Definition 5.7(iii) holds for $(A, G)$. Then $A^{G}$ is cyclotomic Gorenstein, and $G$ is generated by bireflections. By Proposition 6.8(4) for $g \in G$, and Proposition 6.8(6) for $A^{G}$, Definition 5.7(iii) holds for $\left(B, G^{\prime}\right)$. Since $B$ is $\Gamma^{\prime}$-Kac, $B^{G^{\prime}}$ is a complete intersection of GK-type. By Lemma $6.5(3), A^{G}$ is a complete intersection of GK-type, and the assertion follows.

(2) The proof is similar to the proof of part (1).

Definition 6.10. Let $A$ be an AS regular algebra and $\Gamma$ be a subgroup of $\operatorname{Aut}(A)$. We say that $A$ is $\Gamma$-Shephard-Todd-Chevalley (or $\Gamma-S T C$ for short) if, for every finite subgroup $G \subset \Gamma$, the fixed subring $A^{G}$ is AS regular if and only if $G$ is generated by reflections of $A$.

Proposition 6.11. Let $A$ be a filtered algebra such that $B:=\operatorname{gr}_{F} A$ is a noetherian connected graded $A S$ regular algebra. Let $\Gamma$ be a subgroup of $\operatorname{Aut}(A)$, and let $\Gamma^{\prime}=$ $\Phi(\Gamma)$. If $B$ is $\Gamma^{\prime}-S T C$, then $A$ is $\Gamma-S T C$.

Proof. Let $G$ be a finite subgroup of $\Gamma$ such that $G$ is generated by reflections. Then $G^{\prime} \subset \Gamma^{\prime}$ is generated by reflections of $B$. Since $B$ is $\Gamma^{\prime}$-STC, $B^{G^{\prime}}$ is AS regular, so by Lemma 6.5 (5), $A^{G}$ is AS regular, proving one direction of the proposition. The other direction follows from [KKZ3, Proposition 2.5(b)].

\section{DOWN-UP ALGEBRAS}

Down-up algebras, denoted by $A(\alpha, \beta, \gamma)$ for $\alpha, \beta, \gamma$ parameters in $k$, were defined by Benkart-Roby $[\overline{B R}]$ and studied by many others. They are $k$-algebras generated by $u$ and $d$, and subject to the relations

$$
\begin{aligned}
& d^{2} u=\alpha d u d+\beta u d^{2}+\gamma d, \\
& d u^{2}=\alpha u d u+\beta u^{2} d+\gamma u .
\end{aligned}
$$

The universal enveloping algebra of the 3-dimensional Heisenberg Lie algebra is $A(2,-1,0)$, and there are other interesting special cases, see [BR, $\mathrm{KK}$. In this paper, we are interested only in the graded case, namely, when $\gamma=0$ (and $\operatorname{deg} u=$ $\operatorname{deg} d=1)$. It is well-known that $A(\alpha, \beta, 0)$ is noetherian if and only if it is AS regular if and only if $\beta \neq 0$ [KMP. See [BW] for some discussion about the representation theory of graded down-up algebras. In this paper $A(\alpha, \beta)$ stands for $A(\alpha, \beta, 0)$.

Throughout the rest of the paper, let $A=A(\alpha, \beta)$ be a noetherian graded downup algebras. We let $a$ and $b$ be the roots of the "character polynomial"

$$
x^{2}-\alpha x-\beta=0,
$$

and let

$$
\Omega_{1}=d u-\text { aud and } \Omega_{2}=d u-b u d .
$$

Note that both $a, b \neq 0$ and $\Omega_{1}=\Omega_{2}$ if and only if $\alpha^{2}=-4 \beta$. 
Lemma 7.1. Both $\Omega_{1}$ and $\Omega_{2}$ are regular normal elements in $A$, and the following relations hold in $A$ :

$$
\begin{aligned}
u \Omega_{1} & =b^{-1} \Omega_{1} u, \\
d \Omega_{1} & =b \Omega_{1} d, \\
u \Omega_{2} & =a^{-1} \Omega_{2} u, \\
d \Omega_{2} & =a \Omega_{2} d, \\
u d \Omega_{i} & =\Omega_{i} u d \text { for } i=1,2, \\
d u \Omega_{i} & =\Omega_{i} d u \text { for } i=1,2, \\
\Omega_{1} \Omega_{2} & =\Omega_{2} \Omega_{1} .
\end{aligned}
$$

Furthermore, the first two equations (as well as the next two equations) are equivalent to the two defining relations of $A$.

Proof. It is easy to check that the first two (as well as the next two) are equivalent to the two defining relations of $A$, and hence $\Omega_{1}$ and $\Omega_{2}$ are normal elements of $A$. Since $A$ is a domain, these are regular elements. The last three equations follow from the first four.

Next we review the graded automorphism groups of $A$, and describe two filtrations of $A$, and their associated graded rings, that will be used later.

Lemma 7.2. Let $A=A(\alpha, \beta)$ with $\beta \neq 0$.

(1) [KK, Proposition 1.1] The graded automorphism group of $A$ is given by

$$
\operatorname{Aut}(A)= \begin{cases}G L_{2}(k) & \text { if }(\alpha, \beta)=(0,1), \\
G L_{2}(k) & \text { if }(\alpha, \beta)=(2,-1), \\
U=\left\{\left(\begin{array}{ll}
a & 0 \\
0 & b
\end{array}\right),\left(\begin{array}{ll}
0 & c \\
d & 0
\end{array}\right): a, b, c, d \in k^{\times}\right\} & \text {if } \beta=-1, \alpha \neq 2, . \\
O=\left\{\left(\begin{array}{ll}
a & 0 \\
0 & b
\end{array}\right): a, b \in k^{\times}\right\} & \text {otherwise. }\end{cases}
$$

(2) Let $\Omega=d u$-aud with $\operatorname{deg} \Omega=2$. Define a filtration $F$ of $A$ by

$$
\begin{aligned}
F(0) & =k, \\
F(-1) & =k+k d+k u+k \Omega=: V, \\
F(-n) & =V^{n}, \forall n>1 .
\end{aligned}
$$

Then $\operatorname{gr}_{F} A$ is isomorphic to the skew polynomial ring

$$
B:=k\langle d, u, \Omega\rangle /(d \Omega=b \Omega d, \Omega u=b u \Omega, d u=a u d) .
$$

(3) Suppose $\beta=-1$ and $\alpha \neq \pm 2$. Let $\Omega_{1}=d u$ - aud and $\Omega_{2}=d u-$ bud with $\operatorname{deg} \Omega_{1}=\operatorname{deg} \Omega_{2}=2$. Define a filtration $F$ by

$$
\begin{aligned}
F(0) & =k, \\
F(-1) & =k+k d+k u+k \Omega_{1}+k \Omega_{2}=: V, \\
F(-n) & =V^{n}, \forall n>1 .
\end{aligned}
$$

Then $\operatorname{gr}_{F} A$ is isomorphic to the algebra

$$
B:=(k[d, u] /(d u))\left\langle\Omega_{1}, \Omega_{2}\right\rangle /(\text { relations })
$$


where relations are

$d \Omega_{1}=b \Omega_{1} d, \Omega_{1} u=b u \Omega_{1}, d \Omega_{2}=a \Omega_{2} d, \Omega_{2} u=a u \Omega_{2},\left[\Omega_{1}, \Omega_{2}\right]=0$.

Proof. The proofs of (2) and (3) are similar, so we give only the proof of (3).

The elements of $\operatorname{gr}_{F}(A)$ satisfy the relations of $B$ so there is an algebra map from $\operatorname{gr}_{F}(A)$ onto $B$. We can filter elements of $A$ by ordered pairs $\mathbb{N} \times \mathbb{N}$, where the first degree is the degree in the filtration $F$ and the second degree is the natural degree in the graded ring $A$ (so that $\operatorname{deg}(u)=\operatorname{deg}(d)=(1,1)$ and $\operatorname{deg}\left(\Omega_{i}\right)=(1,2)$ ). Considering the grading in second component, $\operatorname{gr}_{F}(A)$ is a graded ring with the same Hilbert series as $A$ under the natural grading $(\operatorname{deg}(u)=\operatorname{deg}(d)=1)$. Under this grading $B$ is a graded ring with Hilbert series

$$
\frac{1-t^{2}}{\left(1-t^{2}\right)^{2}(1-t)^{2}}
$$

which is the same as the Hilbert series of $\operatorname{gr}_{F}(A)$. Hence the map from $\operatorname{gr}_{F}(A)$ to $B$ is an isomorphism.

Lemma 7.3. Let $A=A(\alpha, \beta)$ with $\beta \neq 0$ and $g$ be an element in $\operatorname{Aut}(A) \subset$ $G L_{2}(k)$.

(1) $\mathrm{KK}$, Theorem 1.5] If $\lambda$ and $\mu$ are eigenvalues of $g$ as a matrix in $G L_{2}(k)$, then the trace of $g$ is

$$
\operatorname{Tr}(g, t)=\frac{1}{(1-\lambda t)(1-\mu t)\left(1-\lambda \mu t^{2}\right)}
$$

(2) [KK, Theorem 1.5] The homological determinant of $g$ is

$$
\text { hdet } g=\lambda^{2} \mu^{2}=(\operatorname{det} g)^{2} \text {. }
$$

As a consequence, if $H$ is a finite subgroup of $\operatorname{Aut}(A)$ with trivial homological determinant, then either $H$ is a finite subgroup of $S L_{2}(k)$, or $H$ satisfies the short exact sequence (E3.0.1).

(3) $g$ is a bireflection of $A$ if and only if either $\operatorname{det} g=1$ (i.e., $\lambda \mu=1$ ) or $g \in G L_{2}(k)$ is a classical reflection (i.e., either $\lambda=1$ or $\mu=1$ ).

(4) Let $B=A\left(\alpha^{\prime}, \beta^{\prime}\right)$ be another down-up algebra. If $G \subset \operatorname{Aut}(A)$ and $G \subset$ $\operatorname{Aut}(B)$, where both $\operatorname{Aut}(A)$ and $\operatorname{Aut}(B)$ are viewed as subgroups of $G L_{2}(k)$. Then $A^{G}$ is AS Gorenstein (respectively, cyclotomic Gorenstein) if and only if $B^{G}$ is.

Proof. (3) This follows from the definition and part (1).

(4) By Stanley's Theorem [JoZ, Theorem 6.1], the property that $A^{G}$ is AS Gorenstein is dependent only on the Hilbert series of $A^{G}$. By Molien's Theorem, the Hilbert series of $A^{G}$ is dependent only on the trace $\operatorname{Tr}(g, t)$ for all $g \in G$. By part (1), $\operatorname{Tr}(g, t)$ is dependent only on the eigenvalues of $g$. Hence the AS Gorenstein property of $A^{G}$ is dependent only on the matrix properties of $G$. Therefore $A^{G}$ is AS Gorenstein if and only if $B^{G}$ is.

By definition, the cyclotomic property is dependent only on the Hilbert series of $A^{G}$, which is again dependent only on the matrix properties of $G$. Therefore $A^{G}$ is cyclotomic Gorenstein if and only if $B^{G}$ is.

See also Proposition 0.2 for further properties of $A$. In particular, $A^{H}$ is $\mathrm{AS}$ Gorenstein if and only if $H$ has trivial homological determinant. To prove Theorem 
0.3 we need analyze all finite subgroups $H \subset \operatorname{Aut}(A)$ with hdet $=1$. We start with some examples in the next section.

\section{AS Gorenstein FIXED SUBRINGS FOR DOWN-UP ALGEBRAS}

In this section, for some values of the parameters $\alpha$ and $\beta$, we discuss the fixed subrings $A^{G}$, where $A$ is a noetherian graded down-up algebra, and $G$ is one of the groups $Q_{i}, i=1, \cdots, 8$, that were introduced in Section 3. All these invariant subrings $A^{G}$ are AS Gorenstein by Lemma 7.3(2) and Proposition 0.2(2), and our interest is in whether the $A^{G}$ are complete intersections of some type. In some cases we are able to express the algebra explicitly as a classical complete intersection. The down-up algebras $A(-1,2)$ and $A(0,1)$, whose graded automorphism groups are all of $G L_{2}(k)$, will be discussed in Section 9 .

Let $A$ be a noetherian graded down-up algebra $A(\alpha, \beta)$ where $\beta \neq 0$. Let $a$ and $b$ be the roots of "character polynomial"

$$
x^{2}-\alpha x-\beta=0 .
$$

Throughout most of this section we assume that $a \neq b$ (i.e. $\alpha^{2} \neq-4 \beta$ ); at the end of the section we consider $A(-2,-1)^{Q_{i}}$ for $i=5, \ldots, 8$. Recall the normal elements $\Omega_{1}:=d u-a u d$ and $\Omega_{2}:=d u-b u d$, which are distinct when $a \neq b$. Below is a table that summarizes the results of this section.

\begin{tabular}{|c|c|c|c|c|}
\hline Group $Q_{i}$ & $A^{Q_{i}}$ & $\begin{array}{l}\text { Generated by } \\
\text { Bireflections? }\end{array}$ & Cyclotomic? & Reference \\
\hline$Q_{1}=\left\langle c_{\epsilon}\right\rangle$ & hypersurface & yes & yes & Example 8.2 \\
\hline $\begin{array}{c}Q_{2}=\left\langle d_{1}, c_{\epsilon}\right\rangle \\
n \text { even }\end{array}$ & $\mathrm{cci}$ & yes & yes & Example 8.3 \\
\hline $\begin{array}{c}Q_{3}=\left\langle d_{1}, c_{\epsilon}\right\rangle \\
n \text { odd }\end{array}$ & $\begin{array}{c}n=1 \text { cci } \\
n \geq 3 \text { no type of ci }\end{array}$ & $\begin{array}{l}\text { yes } \\
\text { yes }\end{array}$ & $\begin{array}{l}\text { yes } \\
\text { no }\end{array}$ & Lemma 8.4 \\
\hline$Q_{4}=\left\langle d_{1}, c_{\epsilon,-}\right\rangle$ & no type of ci & no & no & Lemma 8.7 \\
\hline $\begin{array}{c}Q_{5}=\left\langle s_{1}, c_{\epsilon}\right\rangle \\
n \text { even }\end{array}$ & gci & yes & yes & Proposition 8.8 \\
\hline$Q_{6}=\left\langle s, c_{\epsilon}\right\rangle$ & gci & yes & yes & Proposition 8.8 \\
\hline $\begin{array}{c}Q_{7}=\left\langle d_{1}, s, c_{\epsilon}\right\rangle \\
n \text { even }\end{array}$ & gci & yes & yes & Proposition 8.8 \\
\hline $\begin{array}{c}Q_{8}=\left\langle s, c_{\epsilon,-}\right\rangle \\
n=4 k\end{array}$ & gci & yes & yes & Proposition 8.8 \\
\hline
\end{tabular}

Table 4: Invariants of down-up algebras with $a \neq b$ under groups $Q_{i}, i=1, \ldots, 8$, and $A(-2,-1)$ for $Q_{i}, i=5, \ldots, 8$.

The following lemma follows by a straightforward induction. 
Lemma 8.1. Let $\Omega_{1}$ and $\Omega_{2}$ be defined as above. Let $n$ be a positive integer. Then

$$
\begin{aligned}
u d & =\frac{1}{b-a}\left(\Omega_{1}-\Omega_{2}\right), \\
d u & =\frac{1}{b-a}\left(b \Omega_{1}-a \Omega_{2}\right), \\
u^{n} d^{n} & =f_{n}\left(\Omega_{1}, \Omega_{2}\right), \\
d^{n} u^{n} & =g_{n}\left(\Omega_{1}, \Omega_{2}\right),
\end{aligned}
$$

where

$$
f_{n}(X, Y)=\frac{\prod_{i=1}^{n}\left(b^{-(i-1)} X-a^{-(i-1)} Y\right)}{(b-a)^{n}}, \quad g_{n}(X, Y)=\frac{\prod_{i=1}^{n}\left(b^{i} X-a^{i} Y\right)}{(b-a)^{n}} .
$$

Furthermore, $f_{n}\left(b^{n} X, a^{n} Y\right)=g_{n}(X, Y)$.

Let $Q_{1} \subset O$ be the cyclic group $C_{n}$ generated $c_{\epsilon}$, where $\epsilon$ is a primitive $n$th root of unity, see Lemma 3.4(1).

Example 8.2. The invariant subring $A^{Q_{1}}$ is generated by

$$
X_{1}:=u^{n}, X_{2}:=d^{n}, X_{3}:=\Omega_{1} \text {, and } X_{4}:=\Omega_{2}
$$

subject to the relations

$$
\begin{array}{ll}
r_{1}: & X_{4} X_{3}=X_{3} X_{4}, \\
r_{2}: & X_{4} X_{2}=a^{-n} X_{2} X_{4}, \\
r_{3}: & X_{4} X_{1}=a^{n} X_{1} X_{4}, \\
r_{4}: & X_{3} X_{2}=b^{-n} X_{2} X_{3}, \\
r_{5}: & X_{3} X_{1}=b^{n} X_{1} X_{3}, \\
r_{6}: & X_{2} X_{1}=X_{1} X_{2}+g_{n}\left(X_{3}, X_{4}\right)-f_{n}\left(X_{3}, X_{4}\right), \\
r_{7}: & X_{1} X_{2}=f_{n}\left(X_{3}, X_{4}\right) .
\end{array}
$$

The first six relations define an iterated Ore extension that is a connected graded, AS regular algebra $C_{1}:=k\left\langle X_{1}, \cdots, X_{4}\right\rangle /\left(r_{1}, \cdots, r_{6}\right)$. The element

$$
\Theta:=X_{1} X_{2}-f_{n}\left(X_{3}, X_{4}\right)
$$

is a regular central element in $C_{1}$ and $A^{Q_{1}} \cong C_{1} /(\Theta)$, and hence $A^{Q_{1}}$ is a hypersurface.

Proof. Using the fact that $f_{n}\left(b^{n} X, a^{n} Y\right)=g_{n}(X, Y)$, it follows that $\Theta$ is central. By relation $r_{7}$ there is a graded surjection from $C_{1} /(\Theta) \rightarrow A^{Q_{1}}$. We show this map is a graded isomorphism by showing these algebras have the same Hilbert series.

The Hilbert series of $C_{1} /(\Theta)$ is

$$
H_{C_{1} /(\Theta)}(t)=\frac{1-t^{2 n}}{\left(1-t^{n}\right)^{2}\left(1-t^{2}\right)^{2}}
$$

while using Molien's Theorem and Lemma 7.3(1), it follows that the Hilbert series of $A^{Q_{1}}$ is

$$
\frac{1}{n} \sum_{i=0}^{n-1} \frac{1}{\left(1-\epsilon^{i} t\right)\left(1-\epsilon^{-i} t\right)\left(1-t^{2}\right)}=h(t) \frac{1}{\left(1-t^{2}\right)} .
$$


But $h(t)$ is the Hilbert series of $k\left[t_{1}, t_{2}\right]^{Q_{1}}$, and it follows from the description of $\left(A_{n}\right)$ that precedes Lemma 3.1 that

$$
H_{k\left[t_{1}, t_{2}\right]^{Q_{1}}}(t)=\frac{1-t^{2 n}}{\left(1-t^{n}\right)^{2}\left(1-t^{2}\right)} .
$$

Hence $H_{A^{Q_{1}}}(t)=H_{C_{1} /(\Theta)}(t)$. As a consequence, $A^{Q_{1}} \cong C_{1} /(\Theta)$.

Let $Q_{2} \subset O$ be the group of order $4 n$, which is generated by $d_{1}$ and $c_{\epsilon}$ for a primitive $2 n$th root of unity $\epsilon$, that occurred in Lemma $3.4(2)$. Let $\tilde{f}_{2 n}\left(x_{1}, x_{2}, x_{3}\right)$ be a (commutative) polynomial in $x_{1}, x_{2}, x_{3}$ such that

$$
f_{2 n}(X, Y)=\tilde{f}_{2 n}\left(X^{2}, X Y, Y^{2}\right) \text {. }
$$

Let $\tilde{g}_{2 n}\left(x_{1}, x_{2}, x_{3}\right)$ be the polynomial defined by

$$
\tilde{g}_{2 n}\left(x_{1}, x_{2}, x_{3}\right)=\tilde{f}_{2 n}\left(b^{4 n} x_{1}, a^{2 n} b^{2 n} x_{2}, a^{4 n} x_{3}\right) .
$$

Then $g_{2 n}(X, Y)=\tilde{g}_{2 n}\left(X^{2}, X Y, Y^{2}\right)$.

Example 8.3. The invariant subring $A^{Q_{2}}$ is generated by

$$
X_{1}:=u^{2 n}, X_{2}:=d^{2 n}, X_{3}:=\Omega_{1}^{2}, X_{4}:=\Omega_{1} \Omega_{2} \text {, and } X_{5}:=\Omega_{2}^{2},
$$

and subject to the relations

$$
\begin{aligned}
r_{1}: & X_{5} X_{4}=X_{4} X_{5}, \\
r_{2}: & X_{5} X_{3}=X_{3} X_{5}, \\
r_{3}: & X_{5} X_{2}=a^{-4 n} X_{2} X_{5}, \\
r_{4}: & X_{5} X_{1}=a^{4 n} X_{1} X_{5}, \\
r_{5}: & X_{4} X_{3}=X_{3} X_{4}, \\
r_{6}: & X_{4} X_{2}=a^{-2 n} b^{-2 n} X_{2} X_{4}, \\
r_{7}: & X_{4} X_{1}=a^{2 n} b^{2 n} X_{1} X_{4}, \\
r_{8}: & X_{3} X_{2}=b^{-4 n} X_{2} X_{3}, \\
r_{9}: & X_{3} X_{1}=b^{4 n} X_{1} X_{3}, \\
r_{10}: & X_{2} X_{1}=X_{1} X_{2}+\tilde{g}_{2 n}\left(X_{3}, X_{4}, X_{5}\right)-\tilde{f}_{2 n}\left(X_{3}, X_{4}, X_{5}\right), \\
r_{11}: & X_{1} X_{2}=\tilde{f}_{2 n}\left(X_{3}, X_{4}, X_{5}\right), \\
r_{12}: & X_{3} X_{5}=X_{4}^{2} .
\end{aligned}
$$

The first ten relations define a connected graded, AS regular algebra

$$
C_{2}:=k\left\langle X_{1}, \cdots, X_{5}\right\rangle /\left(r_{1}, \cdots, r_{10}\right)
$$

that is an iterated Ore extension. The elements

$$
\Theta_{1}:=X_{1} X_{2}-\tilde{f}_{2 n}\left(X_{3}, X_{4}, X_{5}\right) \quad \text { and } \Theta_{2}:=X_{4}^{2}-X_{3} X_{5}
$$

form a sequence of regular normalizing elements in $C_{2}$ and $A^{Q_{2}} \cong C_{2} /\left(\Theta_{1}, \Theta_{2}\right)$. As a consequence, $A^{Q_{2}}$ is a cci.

Proof. It is not hard to check that $\Theta_{2}$ is normal in $C_{2}$. The element $\Theta_{1}$ is central in $C_{2}$ because $\tilde{g}_{2 n}\left(x_{1}, x_{2}, x_{3}\right)=\tilde{f}_{2 n}\left(b^{4 n} x_{1}, a^{2 n} b^{2 n} x_{2}, a^{4 n} x_{3}\right)$ implies

$$
X_{i} \tilde{g}_{2 n}\left(x_{1}, x_{2}, x_{3}\right)=\tilde{f}_{2 n}\left(x_{1}, x_{2}, x_{3}\right) X_{i}
$$


for $\mathrm{i}=1,2$, since both sides of the equation have the same monomial summands; furthermore, $X_{3}, X_{4}$ and $X_{5}$ clearly commute with $\Theta_{1}$, so that $\Theta_{1}$ is central in $C_{2}$. Clearly $\Theta_{2}$ is a nonzero element of a domain, and $\Theta_{1}$ is regular in $C_{2} /\left(\Theta_{2}\right)$ because its leading term does not contain $X_{3}, X_{4}$ or $X_{5}$. Hence $\Theta_{2}, \Theta_{1}$ is a regular normalizing sequence.

There is a graded homomorphism from $B:=C_{2} /\left(\Theta_{1}, \Theta_{2}\right)$ onto $A^{Q_{2}}$, since the elements $X_{i}$ satisfy the relations in $B$. We show that this map is injective by showing that $B$ and $A^{Q_{2}}$ have the same Hilbert series. The Hilbert series of $B$ is

$$
H_{B}(t)=\frac{\left(1-t^{4 n}\right)\left(1-t^{8}\right)}{\left(1-t^{2 n}\right)^{2}\left(1-t^{4}\right)^{3}}=\frac{\left(1-t^{4 n}\right)\left(1+t^{4}\right)}{\left(1-t^{2 n}\right)^{2}\left(1-t^{4}\right)^{2}} .
$$

By Molien's Theorem and Lemma $7.3(1)$ the Hilbert series of $A^{Q_{2}}$ is

$$
\begin{aligned}
H_{A^{Q_{2}}}(t) & =\frac{1}{4 n}\left(\sum_{i=0}^{2 n-1} \frac{1}{\left(1-\epsilon^{i} t\right)\left(1-\epsilon^{-i} t\right)\left(1-t^{2}\right)}+\sum_{i=0}^{2 n-1} \frac{1}{\left(1+\epsilon^{i} t\right)\left(1-\epsilon^{-i} t\right)\left(1+t^{2}\right)}\right) \\
& :=\frac{1}{4 n}\left(S_{1} \frac{1}{\left(1-t^{2}\right)}+S_{2} \frac{1}{\left(1+t^{2}\right)}\right),
\end{aligned}
$$

where $S_{1}$ and $S_{2}$ are the indicated sums. We can compute $S_{1}$ and $S_{2}$ from the commutative case, using the fact that $k\left[t_{1}, t_{2}\right]^{Q_{2}}$ is generated by $t_{1}^{2 n}, t_{2}^{2 n}, t_{1}^{2} t_{2}^{2}$, which is the hypersurface $k\left[Z_{1}, Z_{2}, Z_{3}\right] /\left(Z_{1} Z_{2}-Z_{3}{ }^{n}\right)$, so has Hilbert series

$$
H_{k\left[t_{1}, t_{2}\right]^{Q_{2}}}(t)=\frac{1-t^{4 n}}{\left(1-t^{2 n}\right)^{2}\left(1-t^{4}\right)},
$$

which by Molien's Theorem is

$$
H_{k\left[t_{1}, t_{2}\right]^{Q_{2}}}(t)=\frac{1}{4 n}\left(S_{1}+S_{2}\right) .
$$

We can compute $S_{1}$ from the Hilbert series of $k\left[t_{1}, t_{2}\right]^{\left(c_{\epsilon}\right)}$, where $\left(c_{\epsilon}\right)$ is the cyclic group of order $2 n$, and Molien's Theorem, and we obtain

$$
S_{1}:=\sum_{i=0}^{2 n-1} \frac{1}{\left(1-\epsilon^{i} t\right)\left(1-\epsilon^{-i} t\right)}=\frac{2 n\left(1-t^{4 n}\right)}{\left(1-t^{2 n}\right)^{2}\left(1-t^{2}\right)} .
$$

We compute $S_{2}$ using the Hilbert series of $k\left[t_{1}, t_{2}\right]^{Q_{2}}$, Molien's Theorem, and $S_{1}$, and we obtain

$$
\begin{aligned}
S_{2} & :=\sum_{i=0}^{2 n-1} \frac{1}{\left(1+\epsilon^{i} t\right)\left(1-\epsilon^{-i} t\right)}=4 n H_{k\left[t_{1}, t_{2}\right]^{Q_{2}}}(t)-S_{1} \\
& =\frac{2 n\left(1-t^{4 n}\right)\left(1-t^{2}\right)}{\left(1-t^{2 n}\right)^{2}\left(1-t^{4}\right)} .
\end{aligned}
$$

Then

$$
\begin{aligned}
H_{A^{Q_{2}}}(t) & =\frac{1}{4 n}\left(S_{1} \frac{1}{\left(1-t^{2}\right)}+S_{2} \frac{1}{\left(1+t^{2}\right)}\right) \\
& =\frac{1}{4 n}\left(\frac{2 n\left(1-t^{4 n}\right)}{\left(1-t^{2 n}\right)^{2}\left(1-t^{2}\right)} \frac{1}{\left(1-t^{2}\right)}+\frac{2 n\left(1-t^{4 n}\right)\left(1-t^{2}\right)}{\left(1-t^{2 n}\right)^{2}\left(1-t^{4}\right)} \frac{1}{\left(1+t^{2}\right)}\right) \\
& =\frac{\left(1-t^{4 n}\right)\left(1+t^{4}\right)}{\left(1-t^{2 n}\right)^{2}\left(1-t^{4}\right)^{2}},
\end{aligned}
$$

completing the proof. 
We next consider the case where we have an odd root of unity.

Lemma 8.4. Let $Q_{3} \subset O$ be the group occurring in Lemma 3.4(3), i.e. the group generated by $d_{1}$ and $c_{\epsilon}$, for $\epsilon$ a primitive $n$th root of unity, for an odd integer $n$.

(1) The group $Q_{3}$ is a bireflection group for $A$.

(2) The invariant subring $A^{Q_{3}}$ is AS Gorenstein.

(3) $A^{Q_{3}}$ is a cci if $n=1$.

(4) $A^{Q_{3}}$ is not cyclotomic if $n>1$.

Proof. (1) Both $d_{1}$ and $c_{\epsilon}$ are bireflections of $A$ by Lemma 7.3(3), and so $Q_{3}$ is a bireflection group for $A$.

(2) This follows from Proposition $0.2(2)$, as $(\operatorname{det} g)^{2}=1$ for all $g \in Q_{3}$ and hence hdet $g=1$ by Lemma 7.3(2).

(3) When $n=1, Q_{3}$ is the cyclic group of order 2 generated by $d_{1}$, and $A^{Q_{3}}$ is generated by

$$
X_{1}:=d, X_{2}=u^{2}, X_{3}:=u(d u-a u d), \text { and } X_{4}:=(d u-a u d)^{2}
$$

subject to the relations

$$
\begin{array}{ll}
r_{1}: & X_{1} X_{2}=a^{2} X_{2} X_{1}+(a+b) X_{3}, \\
r_{2}: & X_{1} X_{3}=a b X_{3} X_{1}+X_{4}, \\
r_{3}: & X_{1} X_{4}=b^{2} X_{4} X_{1}, \\
r_{4}: & X_{2} X_{3}=b^{-2} X_{3} X_{2}, \\
r_{5}: & X_{2} X_{4}=b^{-4} X_{4} X_{2}, \\
r_{6}: & X_{3} X_{4}=b^{-2} X_{4} X_{3}, \\
r_{7}: & X_{2} X_{4}=b^{-1} X_{3}^{2} .
\end{array}
$$

The first six relations define an AS regular algebra $C$. Let $\Omega:=X_{2} X_{4}-b^{-1} X_{3}^{2}$; it is not hard to check that $\Omega$ is normal in $C$, and that there is a graded homomorphism from $C /(\Omega)$ onto $A^{Q_{3}}$. By Molien's Theorem, the Hilbert series of $A^{Q_{3}}$ is

$$
\frac{1}{2}\left(\frac{1}{(1-t)^{2}\left(1-t^{2}\right)}+\frac{1}{(1-t)(1+t)\left(1+t^{2}\right)}\right)=\frac{1-t^{6}}{(1-t)\left(1-t^{2}\right)\left(1-t^{3}\right)\left(1-t^{4}\right)},
$$

which is the Hilbert series of $C /(\Omega)$. Hence $A^{Q_{3}}$ is isomorphic to $C /(\Omega)$, and so $A^{Q_{3}}$ is a cci.

(4) Assume $n>1$. Since $Q_{3} \subset O, Q_{3}$ preserves the filtration $F$ defined in Lemma 7.2 (2). By Proposition 6.8 we need to show only that $\left(\operatorname{gr}_{F} A\right)^{\Phi\left(Q_{3}\right)}$ is not cyclotomic. By Lemma 7.2(2), $\operatorname{gr}_{F} A$ is isomorphic to skew polynomial ring

$$
B:=k\left\langle t_{1}, t_{2}, t_{3}\right\rangle /\left(t_{2} t_{3}=b t_{3} t_{2}, b t_{1} t_{3}=t_{3} t_{1}, t_{2} t_{1}=a t_{1} t_{2}\right),
$$

where $t_{1}:=\widehat{u}, t_{2}:=\widehat{d}$ and $t_{3}:=\widehat{\Omega}$. Hence $\operatorname{deg} t_{1}=\operatorname{deg} t_{2}=1, \operatorname{deg} t_{3}=2$, and the group $\Phi\left(Q_{3}\right) \subset \operatorname{Aut}(B)$ is generated by $\widehat{d}_{1}: t_{1} \rightarrow-t_{1}, t_{2} \rightarrow t_{2}, t_{3} \rightarrow-t_{3}$ and $\widehat{c_{\epsilon}}: t_{1} \rightarrow \epsilon t_{1}, t_{2} \rightarrow \epsilon^{-1} t_{2}, t_{3} \rightarrow t_{3}$. Let $G$ be the subgroup of $\Phi\left(Q_{3}\right)$ generated by $\widehat{c_{\epsilon}}$. Then $B^{G}$ is isomorphic to the algebra $S:=\left(k_{p_{i j}}\left[X_{1}, X_{2}, X_{3}\right] /(f)\right)\left[t_{3} ; \sigma\right]$, where $X_{1}:=t_{1}^{n}, X_{2}:=t_{2}^{n}, X_{3}:=t_{1} t_{2}$ and $f:=X_{2} X_{1}-a^{\left(\begin{array}{c}n \\ 2\end{array}\right)} X_{3}^{n}$ (for some appropriate $p_{i j}$ 's and $\sigma$, which are not essential for the argument). The automorphism $\widehat{d_{1}}$ induces an automorphism $h \in \operatorname{Aut}(S)$, which is determined by

$$
h: X_{1} \rightarrow-X_{1}, X_{2} \rightarrow X_{2}, X_{3} \rightarrow-X_{3}, t_{3} \rightarrow-t_{3} .
$$


By Lemma 1.6(3), the trace of $h$ is

$$
\operatorname{Tr}_{S}(h, t)=\frac{1+t^{2 n}}{\left(1+t^{n}\right)\left(1-t^{n}\right)\left(1+t^{2}\right)\left(1+t^{2}\right)},
$$

and the Hilbert series of $S$ is

$$
H_{S}(t)=\operatorname{Tr}_{S}\left(I d_{S}, t\right)=\frac{1-t^{2 n}}{\left(1-t^{n}\right)\left(1-t^{n}\right)\left(1-t^{2}\right)\left(1-t^{2}\right)} .
$$

Since $h$ is an involution, by Molien's theorem,

$$
\begin{aligned}
H_{A^{Q_{3}}}(t) & =H_{B^{\Phi}\left(Q_{3}\right)}(t)=H_{S^{h}}(t) \\
& =\frac{1}{2}\left(\operatorname{Tr}_{S}(h, t)+H_{S}(t)\right) \\
& =\frac{\left(1+t^{n}+t^{2 n}\right)\left(1+t^{4}\right)+2 t^{n+2}}{\left(1-t^{2 n}\right)\left(1-t^{4}\right)^{2}} .
\end{aligned}
$$

By the following lemma, Lemma $8.6(\mathrm{a}),\left(1+t^{n}+t^{2 n}\right)\left(1+t^{4}\right)+2 t^{n+2}$ is not a product of cyclotomic polynomials. Therefore $A^{Q_{3}}$ is not cyclotomic.

Remark 8.5. In the classical case there are groups generated by bireflections, where the fixed ring is not a complete intersection. Let $G$ be the finite subgroup of $S L_{3}(k)$ generated by

$$
\left(\begin{array}{ccc}
\epsilon & 0 & 0 \\
0 & \epsilon^{-1} & 0 \\
0 & 0 & 1
\end{array}\right) \text { and }\left(\begin{array}{ccc}
-1 & 0 & 0 \\
0 & 1 & 0 \\
0 & 0 & -1
\end{array}\right)
$$

where $n$ is a primitive $n$th root of unity for odd integer $n \geq 3$. Both matrices are classical bireflections, so that $G$ is a bireflection group for $k\left[t_{1}, t_{2}, t_{3}\right]$. A Hilbert series argument similar to the one in the proof of the previous lemma shows that $k\left[t_{1}, t_{2}, t_{3}\right]^{G}$ is not cyclotomic, whence, not a complete intersection. (In this case the numerator is $f(t)=t^{2 n}+t^{n+2}+t^{n}+1$ and $t=-1$ is a root; further $f^{\prime}(-1)=2$, so $f(t)$ is increasing at $t=-1$. Since $\lim _{t \rightarrow-\infty} f(t)=\infty$, there must be another real root smaller than -1 (See also WR, Wa $)$.

Lemma 8.6. Each of the following polynomials is not a product of cyclotomic polynomials.

(1) $q(t)=\left(1+t^{n}+t^{2 n}\right)\left(1+t^{4}\right)+2 t^{n+2}$ for any $n>1$.

(2) $q(t)=\left(1+t^{2 n}\right)\left(1+t^{4}\right)+4 t^{n+2}$ for any $n \geq 1$.

Proof. (1) Set $t=e(s):=e^{2 \pi i s}$. Then $f_{n}(t)$ is cyclotomic if and only if $f_{n}(e(s))$ has $2 n+4$ real roots in $[0,1)$. Let

$$
\begin{aligned}
g_{n}(s) & =\frac{e(-(n+2) s)}{4} f_{n}(e(s)) \\
& =\cos (4 \pi s) \cos (2 \pi n s)+\cos ^{2}(2 \pi s)
\end{aligned}
$$

(using the addition formula for $\cos (\alpha+\beta)$ ). Then for $0 \leq s \leq 1 / 8$ we have

$$
\cos (4 \pi s)(\cos (2 \pi n s)+1) \geq 0,
$$

and consequently

$$
g_{n}(s) \geq \sin ^{2}(2 \pi s)>0
$$

for $0<s \leq 1 / 8$. But, for $n>2$, we compute

$$
g^{\prime}(1 / 4 n)=-2 \pi(n \cos (\pi / n)+\sin (\pi / n))<0,
$$


and

$$
g_{n}^{\prime}(1 / 2 n)=2 \pi \sin (2 \pi / n)>0,
$$

so $g_{n}(s)$ has a local minimum at a point $s_{0} \in(1 / 4 n, 1 / 2 n)$, and $g_{n}\left(s_{0}\right)>0$ if $n \geq 4$. Thus $f_{n}(t)$ has a least one root off the unit circle for $n \geq 4$, and one can check the cases $n=2$ and $n=3$.

(2) As in the previous argument, let $t=e(s):=e^{2 \pi i s}$. Then $f_{n}(t)$ is cyclotomic if and only if $f_{n}(e(s))$ has $2 n+4$ real roots in $[0,1)$. Let

$$
\begin{aligned}
g_{n}(s) & =\frac{e(-(n+2) s)}{4} f_{n}(e(s)) \\
& =\cos (4 \pi s) \cos (2 \pi n s)+1 .
\end{aligned}
$$

Hence $s$ is a root of $g_{n}(s)$ if and only if $s$ is a root of $f_{n}(e(s))$, which happens in the following two cases: either $\cos (4 \pi s)=1$ and $\cos (2 \pi n s)=-1$, or $\cos (4 \pi s)=-1$ and $\cos (2 \pi n s)=1$.

Case a: $\cos (4 \pi s)=1$ implies that $s=0$ or $1 / 2$ since $s \in[0,1)$, and $\cos (2 \pi n s)=-1$ implies that $s=1 / 2$ (and this equation holds only if $n$ is odd). In this case, the only possible cyclotomic root of $f_{n}(t)$ can be $e^{\pi i}=-1$.

Case b: $\cos (2 \pi n s)=-1$ implies that $s=1 / 4$ or $3 / 4$, and in either case $\cos (2 \pi n s)=$ 1 implies that $n$ is a multiple of 4 , and the only cyclotomic roots of $f_{n}(t)$ are $\pm i$. Hence if $n$ is odd the only cyclotomic factor can be $t+1$, and for any $n \geq 0$, $f_{n}(t) \neq k(t+1)^{m}$ for any $k \in k$. When $n$ is even (and a multiple of 4 ) the only possible cyclotomic factor is $t^{2}+1$, but for any $n>0 f_{n}(t) \neq k\left(t^{2}+1\right)^{m}$ for any $k \in k$.

Similar to Lemma 8.4 we have the following.

Lemma 8.7. Let $Q_{4} \subset O$ be the group generated by $c_{\epsilon,-}$, for a primitive 4 th root of unity $\epsilon$ for any $n \geq 1$, which occurred in Lemma 3.4(4). The invariant subring $A^{Q_{4}}$ is AS Gorenstein, but not cyclotomic. The group $Q_{4}$ is not a bireflection group.

Proof. Every element in $Q_{4}$ is of the form $c_{\epsilon,-}^{i}$ for $i=0,1, \cdots, 4 n-1$. It is easy to see that the trace of $c_{\epsilon,-}^{i}$ (as an automorphism of $A$ ) is

$$
\operatorname{Tr}_{A}\left(c_{\epsilon,-}^{i}, t\right)=\frac{1}{\left(1-(-1)^{i} \epsilon^{i} t\right)\left(1-\epsilon^{-i} t\right)\left(1-(-1)^{i} t^{2}\right)} .
$$

Hence $c_{\epsilon,-}^{i}$ is a bireflection if and only if $i$ is even, and this implies that $Q_{4}$ is not a bireflection group for $A$.

By Lemma 1.3, one sees that $\operatorname{hdet} c_{\epsilon,-}^{i}=1$ for all $i$. Therefore $A^{Q_{4}}$ is AS Gorenstein [Proposition 0.2(2)]. It remains to show that $A^{Q_{4}}$ is not cyclotomic.

Since $Q_{4} \subset O, Q_{4}$ preserves the filtration $F$ defined in Lemma [7.2(2). By Proposition 6.8 (2) we need to show only that $\left(\operatorname{gr}_{F} A\right)^{\Phi\left(Q_{4}\right)}$ is not cyclotomic. By Lemma 7.2 (2), $\operatorname{gr}_{F} A$ is isomorphic to skew polynomial ring

$$
B:=k\left\langle t_{1}, t_{2}, t_{3}\right\rangle /\left(t_{2} t_{3}=b t_{3} t_{2}, b t_{1} t_{3}=t_{3} t_{1}, t_{2} t_{1}=a t_{1} t_{2}\right)
$$

where $t_{1}:=\widehat{u}, t_{2}:=\widehat{d}$ and $t_{3}:=\widehat{\Omega}$. Hence $\operatorname{deg} t_{1}=\operatorname{deg} t_{2}=1, \operatorname{deg} t_{3}=2$ and the $\operatorname{group} \Phi\left(Q_{4}\right) \subset \operatorname{Aut}(B)$ is generated $\phi:=\Phi\left(c_{\epsilon,-}\right): t_{1} \rightarrow-\epsilon t_{1}, t_{2} \rightarrow \epsilon^{-1} t_{2}, t_{3} \rightarrow-t_{3}$, where $\epsilon$ is a primitive $4 n$th root of unity. Let $G$ be the subgroup of $\Phi\left(Q_{4}\right)$ generated by $\phi^{2}$. Then $B^{G}$ is isomorphic to the algebra $S:=\left(k_{p_{i j}}\left[X_{1}, X_{2}, X_{3}\right] /(f)\right)\left[t_{3} ; \sigma\right]$, where $X_{1}:=t_{1}^{2 n}, X_{2}:=t_{2}^{2 n} X_{3}:=t_{1} t_{2}$, where $p_{12}:=a^{4 n^{2}}, p_{13}:=a^{2 n}$ and $p_{23}:=$ 
$a^{-2 n}$, and where $f:=X_{1} X_{2}-a^{-\left(\begin{array}{c}2 n \\ 2\end{array}\right)} X_{3}^{2 n}$. The automorphism $\phi \in \operatorname{Aut}(B)$ induces an involution $h \in \operatorname{Aut}(S)$, which is determined by

$$
h: X_{1} \rightarrow-X_{1}, \quad X_{2} \rightarrow-X_{2}, \quad X_{3} \rightarrow-X_{3}, \quad t_{3} \rightarrow-t_{3} .
$$

Note that $h(f)=f$. By Lemma 1.6(3), the trace of $h$ is

$$
\operatorname{Tr}_{S}(h, t)=\frac{1-t^{4 n}}{\left(1+t^{2 n}\right)\left(1+t^{2 n}\right)\left(1+t^{2}\right)\left(1+t^{2}\right)},
$$

and the Hilbert series of $S$ is

$$
H_{S}(t)=\operatorname{Tr}_{S}\left(I d_{S}, t\right)=\frac{1-t^{4 n}}{\left(1-t^{2 n}\right)\left(1-t^{2 n}\right)\left(1-t^{2}\right)\left(1-t^{2}\right)} .
$$

Since $h$ is an involution, by Molien's theorem,

$$
\begin{aligned}
H_{A^{Q_{4}}}(t) & =H_{B^{\Phi\left(Q_{4}\right)}}(t)=H_{S^{h}}(t) \\
& =\frac{1}{2}\left(\operatorname{Tr}_{S}(h, t)+H_{S}(t)\right) \\
& =\frac{\left(1-t^{4 n}\right)\left[\left(1+t^{4 n}\right)\left(1+t^{4}\right)+4 t^{2 n+2}\right]}{\left(1-t^{4 n}\right)^{2}\left(1-t^{4}\right)^{2}} .
\end{aligned}
$$

By the previous lemma, Lemma 8.6(b), $\left(1+t^{4 n}\right)\left(1+t^{4}\right)+4 t^{2 n+2}$ is not a product of cyclotomic polynomials. Therefore $A^{Q_{4}}$ is not cyclotomic.

Proposition 8.8. Suppose that $\beta=-1$ and $\alpha \neq 2$. Let $H$ be any of the groups $Q_{5}, Q_{6}, Q_{7}$ or $Q_{8}$ in Lemma 3.4. Then the invariant subring $A^{H}$ is a gci.

Proof. First we assume that $\alpha \neq-2$. Since $\beta=-1$ and $\alpha \neq \pm 2, b=a^{-1} \neq \pm 1$, so that $\Omega_{1}=d u-a u d$ and $\Omega_{2}=d u-a^{-1} u d$ are distinct elements of $A$.

We consider each of the four different groups $Q_{5}, Q_{6}, Q_{7}$, and $Q_{8}$ for $A=$ $A(\alpha,-1)$ when $\alpha \neq-2$. Each of these groups preserves the filtration of Lemma 7.2(3), defined by $F(n)=V^{-n}$, where $V=k d+k u+k \Omega_{1}+k \Omega_{2}$, and the associated graded ring is isomorphic to

$$
B:=\left(k\left[t_{1}, t_{2}\right] /\left(t_{1} t_{2}\right)\right)\left\langle\Omega_{1}, \Omega_{2}\right\rangle /(\text { relations }),
$$

where relations are

$$
t_{1} \Omega_{1}=a^{-1} \Omega_{1} t_{1}, \Omega_{1} t_{2}=a^{-1} t_{2} \Omega_{1}, t_{1} \Omega_{2}=a \Omega_{2} t_{1}, \Omega_{2} t_{2}=a t_{2} \Omega_{2},\left[\Omega_{1}, \Omega_{2}\right]=0 .
$$

By Proposition $0.4(1)$, in each of the four cases it suffices to show that $B^{\Phi(H)}$ is a gci.

Case (i): $H=Q_{5}$.

Recall that $Q_{5} \subset U$ is the binary dihedral group $B D_{4 n}$ generated $s_{1}$ and $c_{\epsilon}$, where $\epsilon$ is a primitive $2 n$th root of unity. Here we also include the abelian case $B D_{4}$. Let $G$ be the subgroup of $\widehat{H}:=\Phi(H)$ generated by $\widehat{c_{\epsilon}}$ which sends

$$
t_{1} \rightarrow \epsilon t_{1}, \quad t_{2} \rightarrow \epsilon^{-1} t_{2}, \quad \Omega_{1} \rightarrow \Omega_{1}, \quad \Omega_{2} \rightarrow \Omega_{2} .
$$

It is easy to see that, by setting $x_{i}=t_{i}^{2 n}$ for $i=1,2, B^{G}$ is isomorphic to

$$
D:=\left(k\left[x_{1}, x_{2}\right] /\left(x_{1} x_{2}\right)\right)\left\langle\Omega_{1}, \Omega_{2}\right\rangle / \text { (relations) }
$$

where relations are

$$
x_{1} \Omega_{1}=a^{-2 n} \Omega_{1} x_{1}, \Omega_{1} x_{2}=a^{-2 n} x_{2} \Omega_{1}, x_{1} \Omega_{2}=a^{2 n} \Omega_{2} x_{1}, \Omega_{2} x_{2}=a^{2 n} x_{2} \Omega_{2},
$$

and

$$
\left[\Omega_{1}, \Omega_{2}\right]=0
$$


It is easy to check that $D \cong T_{a^{2 n}} /\left(x_{1} x_{2}\right)$, where $T_{a^{2 n}}$ was studied in Example 5.9. By identifying $\Omega_{i} \in D$ with $y_{i} \in T_{a^{2 n}}$ we have $D=T_{a^{2 n}} /\left(x_{1} x_{2}\right)$. The quotient group $\widehat{H} / G$ is generated by the involution $\widehat{s_{1}}$, which sends

$$
x_{1} \rightarrow x_{2}, \quad x_{2} \rightarrow x_{1}, \quad \Omega_{1} \rightarrow a \Omega_{2}, \quad \Omega_{2} \rightarrow a^{-1} \Omega_{1} .
$$

In particular, $\widehat{s_{1}}$ preserves the relation $x_{1} x_{2}=0$. Therefore

$$
B^{\Phi(H)}=D^{\widehat{s_{1}}}=\left(T_{a^{2 n}} /\left(x_{1} x_{2}\right)\right)^{\widehat{s_{1}}} \cong\left(T_{a^{2 n}}\right)^{\widehat{s_{1}}} /\left(x_{1} x_{2}\right),
$$

where the last equation follows from Lemma 5.4(2). Therefore it suffices to show that $\left(T_{a^{2 n}}\right)^{\widehat{s_{1}}}$ is a gci. Now $\widehat{s_{1}}=\zeta_{2}$ as defined in Example 5.9 for $c=a$. By Example 5.9(ii), $\left(T_{a^{2 n}}\right)^{\widehat{s_{1}}}$ is a cci, whence a gci by Lemma 5.3 (1). This finishes the first case.

Case (ii): $H=Q_{6}$.

The first part of argument is similar to the argument for $H=Q_{5}$ (where we replace $2 n$ by $n)$. The quotient group $\widehat{H} / G$ is generated by the involution $\widehat{s}$, which sends

$$
x_{1} \rightarrow x_{2}, \quad x_{2} \rightarrow x_{1}, \quad \Omega_{1} \rightarrow-a \Omega_{2}, \quad \Omega_{2} \rightarrow-a^{-1} \Omega_{1} .
$$

So the difference from the previous case is that $\widehat{s_{1}}=\zeta_{2}$ for $c=-a$ (not $a$ ). Hence $A^{Q_{6}}$ is a gci.

Case (iii): $H=Q_{7}$.

The first part of the proof is similar to the argument above. Recall that $Q_{7} \subset U$ is generated $d_{1}, s$ and $c_{\epsilon}$, where $\epsilon$ is a primitive $2 n$th root of unity. We use again the filtration of Lemma 7.2(3), and let $B$ be the associate graded algebra. Let $G$ be the subgroup of $\widehat{H}:=\Phi(H)$ generated by $\widehat{c_{\epsilon}}$, which sends

$$
t_{1} \rightarrow \epsilon t_{1}, \quad t_{2} \rightarrow \epsilon^{-1} t_{2}, \quad \Omega_{1} \rightarrow \Omega_{1}, \quad \Omega_{2} \rightarrow \Omega_{2} .
$$

Let $x_{i}=t_{i}^{2 n}$ for $i=1,2$. Then $B^{G}=T_{a^{2 n}} /\left(x_{1} x_{2}\right)$ by identifying $x_{i} \in B^{G}$ with $x_{i} \in T_{a^{2 n}}$ and $\Omega_{i} \in B^{G}$ with $y_{i} \in T_{a^{2 n}}$ for $i=1,2$. The quotient group $W:=\widehat{H} / G$ is generated by the involutions

$$
\widehat{s}: x_{1} \rightarrow x_{2}, \quad x_{2} \rightarrow x_{1}, \quad \Omega_{1} \rightarrow-a \Omega_{2}, \quad \Omega_{2} \rightarrow-a^{-1} \Omega_{1},
$$

and

$$
\widehat{d_{1}}: x_{1} \rightarrow x_{1}, \quad x_{2} \rightarrow x_{2}, \quad \Omega_{1} \rightarrow-\Omega_{1}, \quad \Omega_{2} \rightarrow-\Omega_{2} .
$$

Note that $\widehat{d_{1}}=\zeta_{1}$ and $\widehat{s}=\zeta_{2}$. Therefore

$$
B^{\Phi(H)}=\left(B^{G}\right)^{W}=\left(T_{a^{2 n}} /\left(x_{1} x_{2}\right)\right)^{W} \cong\left(T_{a^{2 n}}\right)^{W} /\left(x_{1} x_{2}\right),
$$

where the last equation follows from Lemma $5.4(2)$. By Example $5.9($ iii $)\left(T_{a^{2 n}}\right)^{W}$ is a cci, whence a gci by Lemma 5.3(1). Therefore $B^{\Phi(H)}$ is a gci, and $A^{Q_{7}}$ is a gci.

Case (iv): $H=Q_{8}$.

The argument is similar, but a bit more complicated.

Recall that $Q_{8} \subset U$ is generated $s$ and $c_{\epsilon,-}$, where $\epsilon$ is a primitive $4 n$th root of unity. We again use the filtration given in Lemma $7.2(3)$, and let $B$ be the associated graded algebra. Let $G$ be the subgroup of $\widehat{H}:=\Phi(H)$ generated by $\widehat{c_{\epsilon^{2}}}=\widehat{c_{\epsilon,-}^{2}}$, which sends

$$
t_{1} \rightarrow \epsilon^{2} t_{1}, \quad t_{2} \rightarrow \epsilon^{-2} t_{2}, \quad \Omega_{1} \rightarrow \Omega_{1}, \quad \Omega_{2} \rightarrow \Omega_{2} .
$$


Let $x_{i}=t_{i}^{2 n}$ for $i=1,2$. Then $B^{G}=T_{a^{2 n}} /\left(x_{1} x_{2}\right)$ by identifying $x_{i} \in B^{G}$ with $x_{i} \in T_{a^{2 n}}$ and $\Omega_{i} \in B^{G}$ with $y_{i} \in T_{a^{2 n}}$ for $i=1,2$. The quotient group $W:=\widehat{H} / G$ is generated by the involutions

$$
\phi_{1}:=\widehat{s}: x_{1} \rightarrow x_{2}, \quad x_{2} \rightarrow x_{1}, \quad \Omega_{1} \rightarrow-a \Omega_{2}, \quad \Omega_{2} \rightarrow-a^{-1} \Omega_{1},
$$

and

$$
\phi_{2}:=\widehat{c_{\epsilon,-}}: x_{1} \rightarrow-x_{1}, \quad x_{2} \rightarrow-x_{2}, \quad \Omega_{1} \rightarrow-\Omega_{1}, \quad \Omega_{2} \rightarrow-\Omega_{2} .
$$

Hence both $\phi_{1}$ and $\phi_{2}$ preserve the relation $x_{1} x_{2}=0$. To show $A^{Q_{8}}$ is a gci, it suffices to show that $B^{\Phi\left(Q_{8}\right)}$ is a gci, or, equivalently, that $\left(B^{G}\right)^{W}=\left(T_{a^{2 n}} /\left(x_{1} x_{2}\right)\right)^{W}$ is. By Lemma 5.4(3), we need to show only that $T_{a^{2 n}}^{W}$ is a gci. Set $q=a^{2 n}$. We need to show that $T_{q}^{W}$ is a gci. Note that $\phi_{1}=\zeta_{1}$ in Example 5.9. By Example 5.9 case (ii), $R:=T_{q}^{\zeta_{2}}$ is isomorphic to $C /(\Theta)$ or $C /\left(\Theta_{1}, \Theta_{2}\right)$. The proofs for these two cases are similar, so let us assume that $R \cong C /\left(\Theta_{1}, \Theta_{2}\right)$, where $C$ and $\Theta_{i}$ are given in Example [5.9] Now $\phi_{2}$ induces an automorphism of $R$ which is equal to

$$
\eta_{2}: X_{1} \rightarrow-X_{1}, \quad X_{2} \rightarrow X_{2}, \quad Y_{1} \rightarrow-Y_{1}, \quad Y_{2} \rightarrow Y_{2}, \quad Z_{1} \rightarrow Z_{1}, \quad Z_{2} \rightarrow Z_{2}
$$

where $X_{1}, X_{2}, Y_{1}, Y_{2}, Z_{1}, Z_{2}$ are the generators of $R$ and $C$. Further, $\eta_{2}$ preserves $\Theta_{1}$ and $\Theta_{2}$. By Lemma 5.4(3), it is enough to show that $C^{\eta_{2}}$ is a gci. One can check that fact directly, or use the filtration to reduce to a simpler case. To illustrate the argument, let us use the filtration. We need to return to the algebra $C$ defined in Example 5.9. One can check easily that $X_{2}$ is a regular normal element. Define

$$
F(n)=\left\{\begin{array}{ll}
x_{2}^{n} C, & n \geq 0 \\
C & n \geq 0
\end{array} .\right.
$$

Then $\eta_{2}$ preserves the filtration. By Proposition $0.4(1)$, it suffices to show that $\left(g_{F} C\right)^{\eta_{2}}$ is a gci. The filtration $F$ gives us $\operatorname{gr}_{F} C=C /\left(X_{2}\right)\left[X_{2} ; \sigma\right]$, for some automorphism $\sigma$. By Lemma 5.4(1), it is enough to show that $\left(C /\left(X_{2}\right)\right)^{\eta_{2}}$ is a gci, where we re-use $\eta_{2}$ for the automorphism of $C /\left(X_{2}\right)$ induced by $\eta_{2}$. We need to return to the algebra in Example 5.9 to see that $Y_{2}$ is a regular normal element in $C /\left(X_{2}\right)$. The same argument shows that we need to show that $\left(C /\left(X_{2}, Y_{2}\right)\right)^{\eta_{2}}$ is a gci. By repeating this argument, the assertion eventually follows from the fact that $\left(C /\left(X_{2}, Y_{2}, Z_{1}, Z_{2}\right)\right)^{\eta_{2}}$ is a gci (which is isomorphic to the second Veronese subring of $\left.k\left[X_{1}, Y_{1}\right]\right)$. This finishes the case when $\alpha \neq-2$.

Next we consider the case when $\alpha=-2$, and $A=A(-2,-1)$. In this case, $a=b=-1$, and we let $\Omega=d u+u d$, and use the filtration defined in Lemma 7.2(2), which is $H$-stable for each of the four groups. The associated graded ring $B:=\operatorname{gr}_{F} A$ is isomorphic to $k_{-1}\left[t_{1}, t_{2}, t_{3}\right]$, where $t_{1}:=\widehat{u}, t_{2}:=\widehat{d}$ and $t_{3}:=\widehat{\Omega}$. By Proposition $0.4(1)$, we need to show only that $B^{\Phi(H)}$ is a gci. Again we have four groups to consider.

Case (i): $H=Q_{5}=B D_{4 n}$.

The group $Q_{5}$ is generated by $s_{1}$ and $c_{\epsilon}$, where $\epsilon$ is a primitive $2 n$th root of unity. Let $G$ be the subgroup of $\widehat{H}:=\Phi(H)$ generated by $\widehat{c_{\epsilon}}$, which sends

$$
t_{1} \rightarrow \epsilon t_{1}, \quad t_{2} \rightarrow \epsilon^{-1} t_{2}, \quad t_{3} \rightarrow t_{3} .
$$

Then $B^{G}$ is isomorphic to

$$
D:=k\left[x_{1}, x_{2}, x_{2}\right] /\left(x_{1} x_{2}-(-1)^{\left(\begin{array}{c}
2 n \\
2
\end{array}\right)} x_{3}^{2 n}\right) \otimes k\left[t_{3}\right],
$$


where $x_{1}=t_{1}^{2 n}, x_{2}=t_{2}^{2 n}$ and $x_{3}=t_{1} t_{2}$. The quotient group $\widehat{H} / G$ is generated by the involution $\widehat{s_{1}}$, which sends

$$
x_{1} \rightarrow x_{2}, \quad x_{2} \rightarrow x_{1}, \quad x_{3} \rightarrow x_{3}, \quad t_{3} \rightarrow-t_{3} .
$$

Now $h:=\widehat{s_{1}}$ satisfies the hypotheses of Lemma 5.5. Therefore $D^{h}$ is a gci, and since $B^{\widehat{H}}=\left(B^{G}\right)^{h}=D^{h}$, we have $B^{\widehat{H}}$, and hence $A^{H}$, is a gci.

Case (ii): $H=Q_{6}$.

The first part of argument is similar to the argument for $H=Q_{5}$ (replacing $2 n$ by $n)$. Let $B=\operatorname{gr}_{F} A=\left(k_{-1}\left[t_{1}, t_{2}\right]\right)\left[t_{3}, \sigma\right]$. Then every $\phi \in H$ preserves $\Omega$, we have $B^{\Phi(H)}=\left(k_{-1}\left[t, t_{2}\right]^{H}\right)\left[t_{3}, \sigma\right]$. Since $H$ has trivial homological determinant when acting on $k_{-1}\left[t, t_{2}\right]$, Theorem 0.1 says that $k_{-1}\left[t, t_{2}\right]^{H}$ is a cci. Thus $B^{\Phi(H)}=\left(k_{-1}\left[t, t_{2}\right]^{H}\right)\left[t_{3}, \sigma\right]$ is a cci, whence a gci, and $A^{H}$ is a gci.

Case (iii): $H=Q_{7}$.

Recall that $Q_{7} \subset U$ is generated by $d_{1}, s$ and $c_{\epsilon}$, where $\epsilon$ is a primitive $2 n$th root of unity. Let $B=\operatorname{gr}_{F} A=k_{-1}\left[t_{1}, t_{2}, t_{3}\right]$ as above. Let $G$ be the subgroup of $\Phi(H)$ generated by $\widehat{c_{\epsilon}}$. Then $B^{G}$ is isomorphic to

$$
D:=k\left[x_{1}, x_{2}, x_{2}\right] /\left(x_{1} x_{2}-(-1)^{\left(\begin{array}{c}
2 n \\
2
\end{array}\right)} x_{3}^{2 n}\right) \otimes k\left[t_{3}\right],
$$

where $x_{1}=t_{1}^{2 n}, x_{2}=t_{2}^{2 n}$ and $x_{3}=t_{1} t_{2}$. The quotient group $W:=\widehat{H} / G$ is generated by the involutions

$$
\phi_{1}:=\widehat{s}: x_{1} \rightarrow x_{2}, \quad x_{2} \rightarrow x_{1}, \quad x_{3} \rightarrow-x_{3}, \quad t_{3} \rightarrow t_{3},
$$

and

$$
\phi_{2}:=\widehat{d_{1}}: x_{1} \rightarrow x_{1}, \quad x_{2} \rightarrow x_{2}, \quad x_{3} \rightarrow-x_{3}, \quad t_{3} \rightarrow-t_{3} .
$$

Since both $\phi_{1}$ and $\phi_{2}$ preserve the relation $f=x_{1} x_{2}-(-1)^{\left(\begin{array}{c}2 n \\ 2\end{array}\right)} x_{3}^{2 n}$, we can lift $\phi_{1}$ and $\phi_{2}$ to the level of $D_{1}:=k\left[x_{1}, x_{2}, x_{3}, t_{3}\right]$. By Lemma 5.4(3), it suffices to show that $D_{1}^{W}$ is a gci. Now $D_{1}$ is commutative, $W$ is a group of order 4 , so it is an easy computation to show that $D_{1}^{W}$ is a cci (see also [G2, $\mathrm{Na}$ ), whence a gci .

Case (iv): $H=Q_{8}$.

Recall that $Q_{8} \subset U$ is generated by $s$ and $c_{\epsilon,-}$, where $\epsilon$ is a primitive $4 n$th root of unity. Let $B=\operatorname{gr}_{F} A=k_{-1}\left[t_{1}, t_{2}, t_{3}\right]$ where $t_{1}:=\widehat{u}, t_{2}:=\widehat{d}$ and $t_{3}:=\widehat{\Omega}$. Let $G$ be the subgroup of $\Phi(H)$ generated by $\widehat{c_{\epsilon^{2}}}$. Then $B^{G}$ is isomorphic to

$$
D:=k\left[x_{1}, x_{2}, x_{2}\right] /\left(x_{1} x_{2}-(-1)^{\left(\begin{array}{c}
2 n \\
2
\end{array}\right)} x_{3}^{2 n}\right) \otimes k\left[t_{3}\right],
$$

where $x_{1}:=t_{1}^{2 n}, x_{2}:=t_{2}^{2 n}$ and $x_{3}:=t_{1} t_{2}$. The quotient group $W:=\widehat{H} / G$ is generated by the involutions

$$
\phi_{1}:=\widehat{s}: x_{1} \rightarrow x_{2}, \quad x_{2} \rightarrow x_{1}, \quad x_{3} \rightarrow-x_{3}, \quad t_{3} \rightarrow t_{3}
$$

and

$$
\phi_{2}: \widehat{c_{\epsilon,-}}: x_{1} \rightarrow-x_{1}, \quad x_{2} \rightarrow-x_{2}, \quad x_{3} \rightarrow-x_{3}, \quad t_{3} \rightarrow-t_{3} .
$$

Both $\phi_{1}$ and $\phi_{2}$ preserves the relation $f:=x_{1} x_{2}-(-1)^{\left(\begin{array}{c}2 n \\ 2\end{array}\right)} x_{3}^{2 n}$. We can lift $\phi_{1}$ and $\phi_{2}$ to the level of $D_{1}:=k\left[x, x_{2}, x_{3}, t_{3}\right]$. By Lemma 5.4 (3), it suffices to show that $D_{1}^{W}$ is a gci. Now $D_{1}$ is commutative, $W$ is a group of order 4 , and it is an easy computation to show that $D_{1}^{W}$ is a cci (see also [G2, $[\mathrm{Na}$ ), whence a gci. This case finishes the argument when $\alpha=-2$. 
Combining all the cases above, the proof is now complete.

\section{Proof of Theorem 0.3}

We are now ready to prove Theorem 0.3 .

Proof of Theorem 0.3. Recall that $A=A(\alpha, \beta)$ is a noetherian graded down-up algebra, and $H$ is a finite subgroup of $\operatorname{Aut}(A)$. By Lemma 5.3 $(2)$, if $A^{H}$ is a gci, then $A^{H}$ is a cyclotomic Gorenstein. To prove Theorem 0.3 one needs to show that, if $A^{H}$ is cyclotomic Gorenstein, then $A^{H}$ is a gci and $H$ is generated by bireflections. This statement is equivalent to the following claim.

Claim 1: For every $H$, either $A^{H}$ is not cyclotomic Gorenstein, or both $A^{H}$ is a gci and $H$ is generated by bireflections.

If $H=Q_{3}$, or is conjugate to $Q_{3}$, and $n>1$, then by Lemma 8.4 there is an $A$ such that $Q_{3} \subset \operatorname{Aut}(A)$ and $A^{Q_{3}}$ is not cyclotomic Gorenstein. By Lemma 7.3(4), if $Q_{3} \subset \operatorname{Aut}(B)$ for any other noetherian graded down-up algebra $B$, then $B^{Q_{3}}$ is not cyclotomic Gorenstein. By the same reasoning, $B^{Q_{3}}$ is cyclotomic Gorenstein when $n=1$, and $Q_{3}$ is generated by bireflections. Similarly, if $Q_{4} \subset \operatorname{Aut}(A)$ for any noetherian graded down-up algebra $A$, then $A^{Q_{4}}$ is not cyclotomic Gorenstein [Lemma 8.7.

Now Claim 1 is equivalent to the following claim.

Claim 2: For every $H$, if $H$ is not conjugate to $Q_{3}$ or $Q_{4}$, then $A^{H}$ is a gci and $H$ is generated by bireflections.

We will analyze $A^{H}$ dependent on $\operatorname{Aut}(A)$, which is dependent on the parameters $(\alpha, \beta)$. First, we present a lemma that deals with some subgroups of $S L_{2}(k)$.

Lemma 9.1. Let $\Omega=d u$ - aud where $a \neq-1$, and let $F$ be the filtration defined in Lemma 7.2(2). Let $H \subset S L_{2}(k)$ be a finite subgroup. Suppose $g(\Omega)=\Omega$ for all $g \in H$. Then $A^{H}$ is a gci.

Proof. Since $g(\Omega)=\Omega$ for all $g \in H$, the filtration given in $7.2(2)$ is $H$-stable. Since $B:=\operatorname{gr}_{F} A$ is a skew polynomial ring $k_{p_{i j}}\left[t_{1}, t_{2}, t_{3}\right]$ that is generated by $t_{1}=\widehat{d}, t_{2}=$ $\widehat{u}$ and $t_{3}=\widehat{\Omega}$, the $\widehat{H}$-action on $B$ fixes $t_{3}$. By Lemma [5.4(1), it suffices to that $k_{a}\left[t_{1}, t_{2}\right]^{H}$ is a gci. Since $H \subset S L_{2}(k)$ and since $a \neq-1, H \subset \operatorname{SLA}\left(k_{a}\left[t_{1}, t_{2}\right]\right)$. By [JoZ, Theorem 3.3], $k_{a}\left[t_{1}, t_{2}\right]^{H}$ is AS Gorenstein. By Theorem $0.1, k_{a}\left[t_{1}, t_{2}\right]^{H}$ is a gci, and therefore the assertion follows.

We note that Lemma 9.1 applies to all values of $\alpha$ and $\beta$, except in the case that all roots of the "character polynomial" are -1 , which happens when

$$
x^{2}-\alpha x-\beta=x^{2}+2 x+1,
$$

hence when $\alpha=-2$ and $\beta=-1$. The argument in the proof of Lemma 9.1 also applies to the case when $H \subset O \cap S L_{2}(k)$ and $a=-1$.

We now use the results we have assembled to prove Theorem 0.3

Case 1: $(\alpha, \beta)=(2,-1)$ or $(0,1)$. In these two case $\operatorname{Aut}(A)=G L_{2}(k)$ by Lemma 7.2 (2). In the first case the roots of the character equation are $a=b=1$, and in the second case they are $a=1$ and $b=-1$ 
Let $\Omega=d u-u d$. Then $g(\Omega)=(\operatorname{det} g) \Omega$ for all $g \in G L_{2}(k)$. If $H$ is a subgroup of $S L_{2}(k)$, Lemma 9.1 shows that $A^{H}$ is always a gci, and Lemma 7.3(3) shows that all elements $(\neq \mathbb{I})$ of $H$ are bireflections, so Claim 2 follows in this case. If $H$ is not a subgroup of $S L_{2}(k)$, by Lemma 7.3(2), $H$ satisfies the exact sequence (E3.0.1). If $G:=H \cap S L_{2}(k)$ is cyclic, then $H$ is isomorphic to a group given in $\left(A_{n, 1}\right),\left(A_{n, 2}\right)$ or $\left(A_{n, 5}\right)$. (Note that the groups occurring in $\left(A_{n, 3}\right)$ and $\left(A_{n, 4}\right)$ are $Q_{3}$ and $Q_{4}$, respectively, so have been eliminated). For any of the cases $\left(A_{n, i}\right)$ for $i=1,2,5$, the proof is very similar, so we do only one case, say $\left(A_{n, 5}\right)$. The filtration given in Lemma $\mathbf{7 . 2}(2)$ is $H$-stable for $\Omega=d u-u d$ (since $a=1$ is a root of the character equation in both cases, and $g(\Omega)=(\operatorname{det} g) \Omega)$. By Proposition $0.4(1)$, it suffices to show that $\left(\operatorname{gr}_{F} A\right)^{\Phi(H)}$ is a gci. Now $\operatorname{gr}_{F} A=\left(k\left[t_{1}, t_{2}\right]\right)\left[t_{3} ; \sigma\right]$ and $\left(\operatorname{gr}_{F} A\right)^{\Phi(G)}=\left(k\left[t_{1}, t_{2}\right]^{G}\right)\left[t_{3} ; \sigma\right]$. Note that $k\left[t_{1}, t_{2}\right]^{G}$ is the algebra $S$ given in the discussion of case $\left(A_{n, 5}\right)$. Let $g \in H \backslash G$, and let $h$ be the induced action of $g$ on $S$. Then $h$ extends to an action on $S\left[t_{3} ; \sigma\right]$ by $h\left(t_{3}\right)=-t_{3}$. One can check that $\widehat{g}=h$. By Lemma 3.3, the hypotheses in Lemma 5.5 hold. Hence, by Lemma 5.5. $\left(S\left[t_{3} ; \sigma\right]\right)^{h}$ is a gci. Finally,

$$
\left(\operatorname{gr}_{F} A\right)^{\Phi(H)}=\left(\left(\operatorname{gr}_{F} A\right)^{\Phi(G)}\right)^{h}=\left(S\left[t_{3} ; \sigma\right]\right)^{h},
$$

which is now a gci. Combining the above, we have that $A^{H}$ is a gci when $H$ is in $\left(A_{n, 5}\right)$.

A similar argument, using Lemmas 3.3 and 5.5 . works when $G$ is of type $D_{n}$, $E_{6}, E_{7}$, or $E_{8}$, finishing Case 1.

Case 2: $H \subset O$. Then $H$ is listed as in Lemma 3.4.

Since $H$ is not conjugate to $Q_{3}$ and $Q_{4}$. One needs to consider only $Q_{1}$ and $Q_{2}$. An argument as in the proof of Lemma 9.1 establishes the result for $Q_{1}$, and an argument as in the proof of case $\left(A_{n, 5}\right)$ establishes the theorem for $Q_{2}$.

If $\beta \neq-1$, then $\operatorname{Aut}(A)=O$, and hence the theorem is established when $\beta \neq-1$.

Case 3: $\beta=-1$ and $H \subset U$.

By Lemma 3.5, $H$ is isomorphic to $Q_{5}, Q_{6}, Q_{7}$ or $Q_{8}$. The case where $\alpha=2$ is covered in Case 1. The case where $\alpha \neq 2$ is covered in Proposition 8.8.

Hence the proof is complete in all cases.

\section{ACKNOWLEDGMENTS}

The authors thank Michael Mossinghoff from Davidson College for the proof of Lemma 8.6. Wake Forest University undergraduate Brian Smith computed several examples that were useful in this work. E. Kirkman was partially supported by grant \#208314 from the Simons Foundation. J.J. Zhang was partially supported by the US National Science Foundation: NSF grant DMS 0855743.

\section{REFERENCES}

[BR] G. Benkart and T. Roby, Down-up algebras, J. Algebra 209 (1998), 305-344. Addendum, J. Algebra 213 (1999), no. 1, 378.

[BW] G. Benkart and S. Witherspoon, A Hopf structure for down-up algebras, Math. Z. 238 (2001), no. 3, 523-553. 
[Bj] J.-E. Björk, The Auslander condition on Noetherian rings, Séminaire d'Algèbre Paul Dubreil et Marie-Paul Malliavin, 39ème Année (Paris, 1987/1988), 137-173, Lecture Notes in Math., 1404, Springer, Berlin, 1989.

[CE] H. Cartan and S. Eilenberg. Homological algebra. Princeton University Press, Princeton, N. J., 1956. $\mathrm{xv}+390 \mathrm{pp}$.

[CKWZ] K. Chan, E. Kirkman, C. Walton and J.J. Zhang, Quantum binary polyhedral groups and their actions on quantum planes, ArXiv 1303.7203 .

[FHT] Y. Félix, S. Halperin and J.-C. Thomas, Elliptic Hopf algebras, J. London Math. Soc. (2) 43 (1991), no. 3, 545-555.

[G1] N.L. Gordeev, Invariants of linear groups generated by matrices with two nonidentity eigenvalues, Zap. Nauchn. Sem. Leningrad. Otdel. Mat. Inst. Steklov. (LOMI) 114 (1982), 120-130; English translation in J. Soviet Math. 27 (1984), no. 4.

[G2] N.L. Gordeev, Finite linear groups whose algebra of invariants is a complete intersection (Russian), Izv. Akad. Nauk SSR Ser. Mat. 50 (1986), no. 2, 343-392. (English translation: Math. USSR-Inv. 28(1987), no. 2, 335-379.)

[G3] N.L. Gordeev, The Hilbert-Poincaré series for some algebras of invariants of cyclic groups, J. Math. Sciences 116 (2003), no 1, 2961-2971.

[Gu] T.H. Gulliksen, A homological characterization of local complete intersections, Compositio Math. 23 (1971) 251-255.

[JiZ] N. Jing and J.J. Zhang, On the trace of graded automorphisms, J. Algebra 189 (1997), no. $2,353-376$.

[JoZ] P. Jørgensen and J.J. Zhang, Gourmet's guide to Gorensteinness, Adv. Math. 151 (2000), no. 2, 313-345.

$[\mathrm{KW}]$ V. Kac and K. Watanabe, Finite linear groups whose ring of invariants is a complete intersection, Bull, Amer. Math. Soc. (N.S.) 6 (1982), no. 2, 221-223.

[KK] E. Kirkman and J. Kuzmanovich, Fixed subrings of Noetherian graded regular rings, J. Algebra 288 (2005), no. 2, 463-484.

[KKZ1] E. Kirkman, J. Kuzmanovich and J.J. Zhang, Rigidity of graded regular algebras, Trans. Amer. Math. Soc. 360 (2008), 6331-6369.

[KKZ2] E. Kirkman, J. Kuzmanovich and J.J. Zhang, Gorenstein subrings of invariants under Hopf algebra actions, J. Algebra 322 (2009), no. 10, 3640-3669.

[KKZ3] E. Kirkman, J. Kuzmanovich and J.J. Zhang, Shephard-Todd-Chevalley Theorem for Skew Polynomial Rings, Algebr. Represent. Theory 13 (2010) no. 2, 127-158

[KKZ4] E. Kirkman, J. Kuzmanovich and J.J. Zhang, Noncommutative complete intersection, ArXiv 1302.6209.

[KKZ5] E. Kirkman, J. Kuzmanovich and J.J. Zhang, Invariants of (-1)-skew polynomial rings under permutation representation, ArXiv 1305.3973.

[KMP] E. Kirkman, I. Musson, D. Passman, Noetherian down-up algebras, Proc. Amer. Math. Soc. 127 (1999), 3161-3167.

[K11] F. Klein. Ueber binäre Formen mit linearen Transformationen in sich selbst. Math. Ann., 9 (2) (1875) 183-208.

[K12] F. Klein, Vorlesungen über das Ikosaeder und die Aufösung der Gleichungen vom fünften Grade, Birkhauser Verlag, Basel, 1993. Reprint of the 1884 original, Edited, with an introduction and commentary by Peter Slodowy.

[KL] G. Krause and T. Lenagan, "Growth of Algebras and Gelfand-Kirillov Dimension", revised edition, Graduate Studies in Mathematics, Vol. 22, American Mathematical Society, Providence, RI, 2000.

[Le] T. Levasseur, Some properties of noncommutative regular graded rings, Glasgow Math. J. 34 (1992), no. 3, 277-300.

[LvO] H. Li and F. van Oystaeyen, "Zariskian filtrations", $K$-Monographs in Mathematics 2, Kluwer Academic Publishers, Dordrecht, 1996.

[LW] G.J Leuschke and R. Wiegand, "Cohen-Macaulay Representations", Mathematical Surveys and Monographs Vol. 181, American Mathematical Society, Providence, RI, 2012.

[Ma] J.P. May, The Cohomology of Restricted Lie Algebras and of Hopf Algebras, J. Algebra 3 (1966) 123-146.

[MR] J. C. McConnell and J. C . Robson, "Noncommutative Noetherian Rings," Wiley, Chichester, 1987. 
[Mc] J. Mcleary, A User's Guide to Spectral Sequences, Second Edition, Cambridge University Press, Cambridge-New York, 2001.

[Na] H. Nakajima, Quotient singularities which are complete intersections, Manuscripta Math. 48 (1984), no.1-3, 163-187.

[No] E. Noether, Der Endlichkeitssatz der Invarianten endlicher Gruppen, Math. Ann. 77 (1916), 89-92.

[St1] R.P. Stanley, Hilbert functions of graded algebras, Adv. Math. 28 (1978), 57-83.

[St2] R.P. Stanley, Invariants of finite groups and their applications to combinatorics, Bulletin of the AMS, 1 (1979), 475-511.

[Su] M. Suzuki, Group theory. I. Translated from the Japanese by the author, Grundlehren der Mathematischen Wissenschaften [Fundamental Principles of Mathematical Sciences], 247. Springer-Verlag, Berlin-New York, 1982.

[WR $]$ K. Watanabe and D. Rotillon, Invariant subrings of $\mathbb{C}[x, y, z]$ which are complete intersections, Manuscripta Math. 39 (1982), 339-357.

[Wa] K. Watanabe, Invariant subrings which are complete intersections I (Invariant subrings of finite abelian groups), Nagoya Math. J. 77 (1980), 89-98.

Kirkman: Department of Mathematics, P. O. Box 7388, Wake Forest University, WinstON-SALEM, NC 27109

E-mail address: kirkman@wfu.edu

Kuzmanovich: Department of Mathematics, P. O. Box 7388, Wake Forest University, Winston-SALEM, NC 27109

E-mail address: kuz@wfu.edu

Zhang: Department of Mathematics, Box 354350, University of Washington, Seattle, WASHINGTON 98195, USA

E-mail address: zhang@math.washington.edu 